\title{
On the double copy for spinning matter
}

\author{
Yilber Fabian Bautista ${ }^{a, b}$ and Alfredo Guevara ${ }^{a, c, d}$ \\ ${ }^{a}$ Perimeter Institute for Theoretical Physics, \\ 31 Caroline St N, Waterloo, ON N2L 2Y5, Canada \\ ${ }^{b}$ Department of Physics and Astronomy, York University, \\ 4700 Keele St, Toronto, ON M3J 1P3, Canada \\ ${ }^{c}$ Department of Physics and Astronomy, University of Waterloo, \\ 200 University Ave W, Waterloo, ON N2L 3G1, Canada \\ ${ }^{d}$ CECs Valdivia and Departamento de Física, Universidad de Concepción, \\ Casilla 160-C, Concepción, Chile \\ E-mail: ybautistachivata@perimeterinstitute.ca, \\ aguevara@perimeterinstitute.ca
}

ABSTRACT: We explore various tree-level double copy constructions for amplitudes including massive particles with spin. By working in general dimensions, we use that particles with spins $s \leq 2$ are fundamental to argue that the corresponding double copy relations partially follow from compactification of their massless counterparts. This massless origin fixes the coupling of gluons, dilatons and axions to matter in a characteristic way (for instance fixing the gyromagnetic ratio), whereas the graviton couples universally reflecting the equivalence principle. For spin-1 matter we conjecture all-order Lagrangians reproducing the interactions with up to two massive lines and we test them in a classical setup, where the massive lines represent spinning compact objects such as black holes. We also test the amplitudes via $\mathrm{CHY}$ formulae for both bosonic and fermionic integrands. At five points, we show that by applying generalized gauge transformations one can obtain a smooth transition from quantum to classical BCJ double copy relations for radiation, thereby providing a QFT derivation for the latter. As an application, we show how the theory arising in the classical double copy of Goldberger and Ridgway can be naturally identified with a certain compactification of $\mathcal{N}=4$ Supergravity.

Keywords: Scattering Amplitudes, Classical Theories of Gravity

ARXiv EPrint: 1908.11349 


\section{Contents}

1 Introduction 1

2 Double copy from dimensional reduction 4

2.1 The $\frac{1}{2} \otimes \frac{1}{2}$ construction 4

2.1.1 Exempli gratia: the multipole expansion $\quad 10$

$\begin{array}{lll}2.2 & \text { General case with } s, \tilde{s} \leq 1 & 11\end{array}$

2.2.1 Non-universality of dilaton couplings 14

2.2.2 Compton amplitude and the $g$ factor $\quad 15$

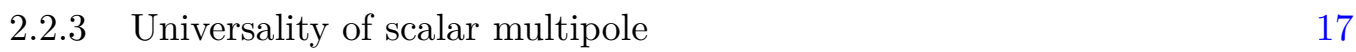

3 Constructing the Lagrangians $\quad 18$

$\begin{array}{lll}3.1 & \text { QCD theories } & 18\end{array}$

$\begin{array}{lll}3.1 .1 & \text { Spins } s=0, \frac{1}{2} & 18\end{array}$

$\begin{array}{lll}3.1 .2 & \text { Spin } s=1 & 20\end{array}$

3.2 Proposal for gravitational theories 23

3.2.1 Alternative construction of the $0 \otimes 1$ action 26

4 Two matter lines from the BCJ construction $\quad 28$

$\begin{array}{lll}4.1 & \text { Elastic scattering } & 30\end{array}$

$\begin{array}{lll}\text { 4.1.1 Spinless case } & 30\end{array}$

4.1.2 Case $s_{a}=0+1$ and $s_{b}=0+0 \quad 31$

4.1.3 Case $s_{a}=s_{b}=0+1 \quad 32$

4.1.4 Case $s_{a}=s_{b}=\frac{1}{2}+\frac{1}{2} \quad 33$

4.2 Inelastic scattering 34

4.2.1 Spinless case 34

4.2.2 Case $s_{a}=s_{b}=\frac{1}{2}+\frac{1}{2} \quad 35$

4.3 Generalized gauge transformations and classical radiation 36

4.3.1 Classical radiation from the standard BCJ double copy 36

$\begin{array}{lll}\text { 4.3.2 Generalized gauge transformation } & 37\end{array}$

$\begin{array}{lll}\text { 4.3.3 Classical limit and Compton residue } & 39\end{array}$

$\begin{array}{lll}\text { 4.3.4 Spinless case } & 40\end{array}$

4.3.5 Case $s_{a}=0+1$ and $s_{b}=0+0 \quad 41$

$\begin{array}{lll}\text { 4.3.6 Case } s_{a}=s_{b}=0+1 & 42\end{array}$

4.3.7 Case $s_{a}=s_{b}=\frac{1}{2}+\frac{1}{2} \quad 43$

5 Discussion 43

$\begin{array}{ll}\text { A Double copy in } d=4 & 45\end{array}$

$\begin{array}{ll}\text { B Tree-level unitarity at } n=4 & 47\end{array}$ 
D Testing amplitudes from CHY-like formulas $\quad 51$

D.1 The $0 \otimes 1$ theory $\quad 51$

D.2 The $\frac{1}{2} \otimes \frac{1}{2}$ theory $\quad 53$

\section{Introduction}

The Bern-Carrasco-Johansson double copy program [1] has demonstrated how certain gravitational quantities can be obtained as a square of gauge-theory ones. Originally introduced for QFT scattering amplitudes with the aim of performing gravitational multiloop computations $[2,3]$, the program has seen many incarnations ranging among the construction of classical space-times [4-12], kinematic algebra realizations [5, 13-16], off-shell extensions [17-19], and more recently applications to gravitational wave phenomena [20-33].

To test the extent of the double copy, and also to study phenomenologically relevant setups, it is desirable to introduce fundamental matter in the construction. This has already been explored in the context of standard QCD [34-39]. Also a number of other interesting cases has been considered, ${ }^{1}$ including quiver theories with bifundamental matter [42-44] and theories with spontaneously broken symmetries [45, 46]. On the other hand the classical double copy, in its many realizations, inherently contains massive matter and hence it is important to clarify the connection between the quantum and classical approaches.

One such step has been taken along refs. [20, 21, 28] which studied gravitational radiation associated to accelerating black holes from the amplitudes point of view. In a recent work [47] we have outlined a direct connection of this phenomena with the spin-multipole expansion, soft theorems and a new operation defining double copy for massive amplitudes with spin. In this work we will thoroughly expand on this latter aspect and show how it arises in a purely QFT framework. We will consider tree-level double copy of massive particles with generic spins and explore several interesting cases.

One of the results of [47] was to obtain graviton-matter amplitudes from double copy at low multiplicities but generic spin quantum number $s$. In order to summarize this in a schematic form, consider a single massive particle of spin- $s$ propagating in a background of photons. We denote the tree-level amplitude involving $n-2$ photons and such a massive line as $A_{n}^{\mathrm{QED}, s}$. Using a symmetric product $\odot$ we then constructed a gravitational amplitude involving one or two gravitons and a massive line, as

$$
A_{n}^{\mathrm{GR}, s+\tilde{s}} \sim A_{n}^{\mathrm{QED}, s} \odot A_{n}^{\mathrm{QED}, \tilde{s}}, \quad n=3,4
$$

where $s+\tilde{s}$ is the spin of the massive line in the graviton amplitude. These amplitudes and their higher multiplicity extensions are relevant for a number of reasons. First, they have

\footnotetext{
${ }^{1}$ For matter-coupled YM theory the gravitational $D=4$ Lagrangians were first obtained from double copy in [40], see also [41].
} 
been recently pinpointed to control the classical limit where the massive lines correspond to compact objects [47-49]. Second, they have been observed to have an exponential form in accord with their multipole expansion [12, 47, 50-52]. Third, they are dimensionindependent and are not polluted with additional states arising from the double copy [47], the latter of which will be evident once we provide the corresponding Lagrangians.

In this paper we will rederive and extend (1.1), mainly focusing on the simplest cases with $s, \tilde{s} \leq 1$. These interactions are fundamental in the sense that they have a healthy high-energy behaviour [53]. By promoting QED to QCD, ${ }^{2}$ studying higher multiplicity amplitudes and the relevant cases for two massive lines, we will identify the gravitational theories obtained by this construction, as promised in [47]. In order to do this we must observe that formula (1.1) has implicit a rather strong assumption, namely the fact that the l.h.s. only depends on the quantum number $s+\tilde{s}$ and not on $s, \tilde{s}$ individually. For instance, this means that for gravitons coupled to a spin-1 field, it should hold that

$$
A_{n}^{\mathrm{GR}, 1} \sim A_{n}^{\mathrm{QCD}, \frac{1}{2}} \odot A_{n}^{\mathrm{QCD}, \frac{1}{2}}=A_{n}^{\mathrm{QCD}, 0} \odot A_{n}^{\mathrm{QCD}, 1},
$$

(we have changed QED to QCD in preparation for $n>4$ ). This means $A_{n}^{\mathrm{gr}, 1}$ not only realizes the equivalence principle in the sense of Weinberg [54] but extends it to deeper orders in the soft expansion $[47,52]$. In the classical limit, the $A_{n}^{\mathrm{gr}, \mathrm{s}}$ amplitudes so constructed will reproduce a well defined compact object irrespective of its double copy factorization. In [47] we exploited condition (1.2) at arbitrary spin to argue that the 3-point amplitude should indeed take an exponential structure, which has recently been identified as a characteristic feature of the Kerr black hole in the sense of [55]. Here we will argue that despite having arbitrary spin, this 3-pt. amplitude can still be considered fundamental as it is essentially equal to its high-energy limit, which in fact implies (1.1)-(1.2).

A simple instance of (1.1) for gravitons was verified explicitly by Holstein [56, 57] (see also [58]) for $s=0, \tilde{s} \leq 1$. He observed that as gravitational amplitudes have an intrinsic gravitomagnetic ratio $g=2$, the double copy (1.1) can only hold by modifying $A_{3}^{\mathrm{QED}, 1}$ away from its "minimal-coupling" value of $g=1$. This modification yields the gyromagnetic ratio $g=2$ characteristic of the electroweak model and was proposed as natural by Weinberg [59]. As observed long ago by Ferrara, Porrati and Telegdi [60] this modification precisely cancels all powers of $1 / \mathrm{m}^{2}$ in $A_{4}^{\mathrm{QED}, 1}$, which otherwise prevented the Compton amplitude to have a smooth high-energy limit. This is a crucial feature, as it hints that the theories with a natural value $g=2$ have a simple massless limit, and indeed can be obtained conversely by compactifying pure massless amplitudes at any multiplicity. Furthermore, it was pointed out in [61] (and recently from a modern perspective [53]) that the appearance of $1 / \mathrm{m}^{2}$ can be avoided up to $s=2$ in the gravitational Compton amplitude $A_{4}^{\mathrm{GR}, s}$ since it corresponds to fundamental interactions. By working on general dimensions, we will see that indeed all such fundamental amplitudes follow from dimensional reduction of massless amplitudes, and ultimately from a compactification of a pure graviton/gluon master amplitude. This is the underlying reason they can be arranged to satisfy (1.1), which in turn simplifies the multipole expansion as we exploited in [47].

\footnotetext{
${ }^{2}$ For the lower multiplicity cases $n=3,4$, one can choose QCD partial amplitudes to coincide with QED amplitudes.
} 
On a different front, it has long been known that the squaring relations in the massless sector yield additional degrees of freedom corresponding to a dilaton $\phi$ and 2-form potential $B_{\mu \nu}$. Their classical counterparts also arise in classical solutions (e.g. string theory backgrounds [62-65]) and therefore emerge naturally (and perhaps inevitably) in the classical double copy $[4,6,20,22]$. It is therefore natural to ask whether the condition (1.2) also holds when the massless states involve such fields. As we have explained this is a non-trivial constraint, and in fact, it only holds for graviton states! To exhibit this phenomena we are led to identify two different gravitational theories, which we refer to as $\frac{1}{2} \otimes \frac{1}{2}$ and $0 \otimes 1$ theories for brevity. The corresponding tree amplitudes will be constructed as

$$
A_{n}^{\frac{1}{2} \otimes \frac{1}{2}} \sim A_{n}^{\mathrm{QCD}, \frac{1}{2}} \otimes A_{n}^{\mathrm{QCD}, \frac{1}{2}}, \quad A_{n}^{0 \otimes 1} \sim A_{n}^{\mathrm{QCD}, 0} \otimes A_{n}^{\mathrm{QCD}, 1}
$$

We conjecture that at all orders in $\kappa=\sqrt{32 \pi G}$ such tree-level interactions follow from the more general Lagrangians,

$$
\frac{\mathcal{L}^{\frac{1}{2} \otimes \frac{1}{2}}}{\sqrt{g}}=-\frac{2}{\kappa^{2}} R+\frac{(d-2)}{2}(\partial \phi)^{2}-\frac{1}{4} e^{\frac{\kappa}{2}(d-4) \phi} F_{\mu \nu}^{I} F_{I}^{\mu \nu}+\frac{m_{I}^{2}}{2} e^{\frac{\kappa}{2}(d-2) \phi} A_{\mu}^{I} A_{I}^{\mu},
$$

and

$$
\begin{aligned}
\frac{\mathcal{L}^{0 \otimes 1}}{\sqrt{g}}= & -\frac{2}{\kappa^{2}} R+\frac{(d-2)}{2}(\partial \phi)^{2}-\frac{e^{-2 \kappa \phi}}{6} H_{\mu \nu \rho}\left(H^{\mu \nu \rho}+\frac{3 \kappa}{2} A_{I}^{\mu} F_{I}^{\nu \rho}\right) \\
& -\frac{1}{4} e^{-\kappa \phi} F_{\mu \nu}^{I} F_{I}^{\mu \nu}+\frac{m_{I}^{2}}{2} A_{\mu}^{I} A_{I}^{\mu}+\text { quartic terms }
\end{aligned}
$$

where $H=d B$ is the field strength of a two-form $B$. Here a sum over $I=1,2$, the flavour index, is implicit and "quartic terms" denote contact interactions between two matter lines that we will identify. These actions will be constructed in general dimensions from simple considerations such as 1) classical behaviour and 2) massless limit/compactification in the string frame. We will then cross-check them against the corresponding QFT amplitudes using modern tools such as massive versions of CHY [66-69] and the connected formalism [70-72]. In the massless limit, the $\frac{1}{2} \otimes \frac{1}{2}$ Lagrangian is known as the Brans-DickeMaxwell (BDM) model with unit coupling [73]. This theory is simpler than $0 \otimes 1$ in many features, for instance in that the $B$-field is not sourced by the matter line and it does not feature quartic interactions. Not surprisingly, in $d=4$ and in the massless limit the $0 \otimes 1$ theory reproduces the bosonic interactions of $\mathcal{N}=4$ Supergravity [74, 75], which is known to arise as the double copy between $\mathcal{N}=4$ Super Yang-Mills (SYM) and pure Yang-Mills $(\mathrm{YM})$ theories $[2,76,77]$. In general dimension we will see that the $0 \otimes 1$ theory is precisely the QFT version of the worldline model constructed by Goldberger and Ridgway in [22, 26] and later extended in $[24,27]$ to exhibit a classical double copy construction with spin. This explains their findings on the fact that the classical double copy not only fixes $g=2$ on the YM side, but also precisely sets the dilaton/axion-matter coupling on the gravity side.

The long-range radiation of a two-body system, emerging in the classical double copy, has been directly linked to a five-point amplitude at leading order [20,31, 47, 78]. We show that by implementing generalized gauge transformations [1] one can define a BCJ gauge 
in which the $\hbar \rightarrow 0$ limit is smooth, i.e. there are no "superclassical" $\sim \frac{1}{\hbar}$ contributions to cancel [78]. The result precisely takes the form derived in [47] from different arguments, i.e. by using the factorization properties of the classical amplitude. This then allows us to translate between the QFT version of the double copy and a classical version of it. We employ this formulae to test double copy in several cases, including the computation of dilaton-axion-graviton radiation with spin [24, 27].

This paper is organized as follows. In section 2 we introduce the double copy for one matter line by studying its massless origin, focusing on the $\frac{1}{2} \otimes \frac{1}{2}$ theory and later extending it in more generality. In section 3 we construct the Lagrangians for both QCD and Gravity from simple arguments, which are then checked against the previous amplitudes. In section 4 we extend both the amplitudes and the Lagrangian construction to two matter lines and define the classical limit to make contact with previous results. In the appendices we provide some further details on the constructions. We also perform checks such as tree-level unitarity and explicit evaluation via the CHY formalism.

Note added. During the final stages of this project we were informed of the publication of [79] which has considered the full spectrum of the $\left(\frac{1}{2}, \frac{1}{2}\right)$ theory in $d=4$ dimensions. In contrast, here we have proposed a consistent tree-level truncation of the matter spectrum which holds in general dimensions. We are grateful to the authors for sharing their manuscript, which led us to include appendix A to show that both constructions are consistent in $d=4$ (see also appendix B). In addition, refs. [49, 80] have appeared, which have focused on scalars and have also employed the compactification to construct the relevant gravitational amplitudes, $A_{n}^{\mathrm{GR}, 0}$.

\section{Double copy from dimensional reduction}

In this section we will introduce the double copy construction by considering a single massive line. In this case one should expect the double copy to hold for massive scalars as their amplitudes can be obtained via compactification of higher dimensional amplitudes [32, $49,58]$. Here we will explicitly demonstrate how this holds even for the case of spinning matter as long as such particles are elementary. This means we consider particles of spin $s \leq 2$ coupled to GR and particles of spin $s \leq 1$ coupled to QCD, in accordance with the notion of [53], see also [61, 81]. The fact that these amplitudes can be chosen to have a smooth high-energy limit can be used backwards to construct them directly from their massless counterparts. On the other hand, once the double copy form of gravitationalmatter amplitudes is achieved one may use it to manifest properties such as the multipole expansion [47]. We will expand on this in section 2.1.1.

\subsection{The $\frac{1}{2} \otimes \frac{1}{2}$ construction}

Let us consider first the case $s=\tilde{s}=\frac{1}{2}$ in (1.1) and relegate the other configurations for the next section. For $D=4$ massless QCD, the double copy procedure was first studied by Johansson and Ochirov [34]. In particular they observed that Weyl-spinors in QCD can be double copied according to the rule $2 \otimes 2=2 \oplus 1 \oplus 1$, where the two new states correspond 
to a photon $\gamma^{ \pm}$and the remaining ones to axion and dilaton scalars. This implies that we can obtain amplitudes in a certain Einstein-Maxwell theory directly from massless QCD. More precisely, for two massive particles we can write

$$
A_{n}^{\frac{1}{2} \otimes \frac{1}{2}}\left(\gamma_{1}^{-} H_{3} \cdots H_{n} \gamma_{2}^{+}\right)=\sum_{\alpha \beta} K_{\alpha \beta}\left[2\left|A_{n, \alpha}^{\mathrm{QCD}}(g \cdots g)\right| 1\right\rangle\left\langle 1\left|\bar{A}_{n, \beta}^{\mathrm{QCD}}(g \cdots g)\right| 2\right],
$$

( $\bar{A}$ here denotes charge conjugation, which will be relevant in the massive case). In the gravitational amplitude the two photon states $\gamma_{1}^{+}, \gamma_{2}^{-}$make a matter line while interacting with the "fat" states $H_{i}$. The latter are obtained from the double copy of the gluons $g_{i}$, and can be taken to be either a Kalb-Ramond field, ${ }^{3}$ a dilaton or a graviton by projecting the product representation into the respective irreps.,

$$
H_{i}^{\mu \nu} \rightarrow \epsilon_{i}^{\mu} \tilde{\epsilon}_{i}^{\nu}=\underbrace{\epsilon_{i}^{[\mu} \tilde{\epsilon}_{i}^{\nu]}}_{B^{\mu \nu}}+\underbrace{\frac{\eta^{\mu \nu}}{D-2} \epsilon_{i} \cdot \tilde{\epsilon}_{i}}_{\eta^{\mu \nu} \frac{\phi}{\sqrt{D-2}}}+\underbrace{\left(\epsilon_{i}^{(\mu} \tilde{\epsilon}_{i}^{\nu)}-\frac{\eta^{\mu \nu}}{D-2} \epsilon_{i} \cdot \tilde{\epsilon}_{i}\right)}_{h^{\mu \nu}} .
$$

The sum over $\alpha, \beta$ in (2.1) ranges over $(n-3)$ ! orderings, where $K_{\alpha, \beta}$ is the standard KLT kernel [82-84]. ${ }^{4}$ This construction can be implemented because for a single matter line we can take the matter particles to be either in the fundamental or in the adjoint representation and the basis of partial amplitudes will be identical [34]. In section 4 we will switch to a more natural prescription for the case of two matter lines.

The r.h.s. of (2.1) exhibits explicitly the helicity weight $\pm \frac{1}{2}$ associated to the Weyl spinors $v_{1}^{-}=|1\rangle$ and $\bar{u}_{2}^{+}=[2 \mid$ of the (massless) matter particles. This means the operators $A^{\mathrm{QCD}}$ and $\bar{A}^{\mathrm{QCD}}$, defined as the amplitude with such spinors stripped, do not carry helicity weight. They can be written as products of Pauli matrices $\sigma^{\mu}, \bar{\sigma}^{\mu}$ where the free Lorentz index is contracted with momenta $p_{i}^{\mu}$ or gluon polarizations $\epsilon_{i}^{\mu}$, as we will see in the examples of the next section. We can alternatively write them in terms of the corresponding spinor-helicity variables as in [35].

Quite generally, the 1.h.s. of (2.1) defines a gauge invariant quantity due to the fact that it is constructed from partial gauge-theory amplitudes. It also has the correct factorization properties (see e.g. $[3,85]$ ). Furthermore, by providing the Lagrangian it will become evident that when the states $H_{i}$ are chosen to be gravitons the amplitude we get for a single matter-line is that of pure Einstein-Maxwell theory, where the dilatons and axions simply decouple. This decoupling is one of the key properties of these objects, which we have exploited in [47]. Similarly, the decoupling of further matter particles will be treated in appendix B.

In order to extend (2.1) to the massive case we rewrite it in a way in which it is not sensitive to the dimension, and then use dimensional reduction. This can be done by introducing polarization vectors for the photons $\gamma^{ \pm}$. Recall that a photon polarization

\footnotetext{
${ }^{3}$ In $D=4$ this field can be dualized to an axion pseudoscalar. We will indistinctly refer to the two-form $B_{\mu \nu}$ as axion or Kalb-Ramond field.

${ }^{4}$ We define the KLT kernel with no coupling constants and absorb the gauge theory coupling $\tilde{g}$ into the generators $\tilde{T}^{a}$ as $\tilde{g} \tilde{T}^{a} \rightarrow T^{a}$. We also absorb the overall factors of $i$ in the definition of the amplitudes and use the conventions for the metric to be in the mostly minus signature.
} 
vector can be taken to be $\epsilon_{\mu}^{+} \sigma^{\mu}=\sqrt{2} \frac{|\mu\rangle[p \mid}{\langle\mu p\rangle}$ where $[\mu \mid$ is a reference spinor carrying the gauge freedom, and analogously $\epsilon_{\mu}^{-} \bar{\sigma}^{\mu}=\sqrt{2} \frac{\mid \mu]\langle p|}{[\mu p]}$. We then have the identity

$$
\begin{aligned}
{[2|X| 1\rangle\langle 1|\bar{Y}| 2] } & =\frac{\left.\operatorname{Tr}\left(X|1\rangle\left[1 \mu_{1}\right]\langle 1|\bar{Y}| 2]\left\langle 2 \mu_{2}\right\rangle \mid 2\right]\right)}{\left[1 \mu_{1}\right]\left\langle 2 \mu_{2}\right\rangle}, \\
& =\frac{1}{2} \operatorname{Tr}\left(X \bar{p}_{1} \epsilon_{1} \bar{Y} p_{2} \bar{\epsilon}_{2}\right),
\end{aligned}
$$

where the bottom line now can be naturally extended to higher dimensions. ${ }^{5}$ It is manifestly gauge invariant since the shift $\epsilon_{i} \rightarrow \epsilon_{i}+p_{i}$ is projected out due to $p_{i} \bar{p}_{i}=0$.

Using this identity, the double copy (2.1) can be uplifted to dimension $D=2 \mathrm{~m}$ as

$$
A_{n}^{\frac{1}{2} \otimes \frac{1}{2}}\left(\gamma_{1} H_{3} \cdots H_{n} \gamma_{2}^{*}\right)=\frac{1}{2} \sum_{\alpha \beta} K_{\alpha \beta} \operatorname{Tr}\left(A_{n, \alpha}^{\mathrm{QCD}}(g \cdots g) \bar{p}_{1} \epsilon_{1} \bar{A}_{n, \beta}^{\mathrm{QCD}}(g \cdots g) p_{2} \bar{\epsilon}_{2}\right) .
$$

Note that the operators $A_{n}^{\mathrm{QCD}}, \bar{A}_{n}^{\mathrm{QCD}}$ in (2.1) are defined under the support of the Dirac equation. This means that they can be shifted by operators proportional to $p_{1}$ or $p_{2}$. The insertion of $p_{1}, p_{2}$ in (2.5) certainly projects out these contributions by using the onshell condition $p \bar{p}=\bar{p} p=0$. For instance, if the matrix operator $A_{n}^{\mathrm{QCD}}$ is shifted by $p_{2 \mu} \sigma^{\mu}$ the QCD amplitude $\bar{u}_{2} A_{n}^{\mathrm{QCD}} v_{1}$ is invariant, and consistently (2.5) picks up no extra contribution, i.e.

$$
\operatorname{Tr}\left(p_{2} \bar{p}_{1} \epsilon_{1} \bar{A}_{n, \beta}^{\mathrm{QCD}}(g \cdots g) p_{2} \bar{\epsilon}_{2}\right)=-\operatorname{Tr}\left(p_{2} p_{2} \bar{p}_{1} \epsilon_{1} \bar{A}_{n, \beta}^{\mathrm{QCD}}(g \cdots g) \epsilon_{2}\right)=0,
$$

where we used $p_{2} \bar{\epsilon}_{2}=-\epsilon_{2} \bar{p}_{2}$. This kind of manipulations are usual when bringing the QCD amplitude into multipole form [47] to make explicit the corresponding form factors.

We now proceed to dimensionally reduce our formulae in order to obtain a KLT expression for massive spin- $\frac{1}{2}$ particles. This follows from a standard KK compactification on a torus, as we explain in the next section. In terms of momenta, we can define the $d=D-1$ components $p_{1}$ and $p_{2}$ via

$$
\begin{aligned}
& P_{1}=\left(m, p_{1}\right), \\
& P_{2}=\left(-m, p_{2}\right), \\
& P_{i}=\left(0, k_{i}\right), \quad i \in\{3, \ldots, n\}
\end{aligned}
$$

which trivially satisfies momentum conservation in the KK component, which we take with minus signature. We also take all momenta outgoing. In terms of Feynman diagrams, the reduction induces the flow of KK momentum through the only path that connects particles $p_{1}$ and $p_{2}$. The propagators in this line are deformed to massive propagators as

$$
\frac{1}{P_{I}^{2}}=\frac{1}{p_{I}^{2}-m^{2}}
$$

\footnotetext{
${ }^{5}$ We represent the Dirac algebra in terms of the $2^{D / 2} \times 2^{D / 2}$ matrices $\Gamma_{D}^{\mu}=\left(\begin{array}{cc}0 & \sigma_{D}^{\mu} \\ \bar{\sigma}_{D}^{\mu} & 0\end{array}\right)$ and define $X=$ $X_{\mu} \sigma_{D}^{\mu}, \bar{X}=X_{\mu} \bar{\sigma}_{D}^{\mu}$ etc. The extension of (2.4) to general dimension simply states that linear combinations $c_{a b} u_{i}^{a} \bar{v}_{i}^{b}$ of the Weyl spinors can be replaced as $c_{a b} v_{i}^{a} \bar{u}_{i}^{b}=p_{i} \bar{\epsilon}_{i}$ for some particular choice of $\epsilon_{i}^{\mu}$ depending on $c_{a b}$. A formula for general dimension is of course obtained by replacing $\sigma^{\mu}, \bar{\sigma}^{\mu} \rightarrow \Gamma^{\mu}$, which in $D=4$ also reduces to $(2.4)$.
} 
where $P_{I}=\left(m, p_{I}\right)$ is the internal momentum. The procedure works straightforwardly when compactifying more particles as long as the KK lines do not cross (i.e. we will not allow interactions between massive particles), as we will explain in the case of two matter lines.

By applying these rules to (2.5) the amplitudes $A^{\text {gr }}, A^{\mathrm{QCD}}$ now contain massive lines and lead to a (gravitational) Proca theory and the massive QCD theory in $d=D-1$ dimensions, respectively. This can be observed easily by applying the dimensional reduction to the Lagrangian as we do in section 3. In the case of the spin-1 theory we choose the polarization vectors $\epsilon_{1}, \epsilon_{2}$ to be $d$-dimensional, i.e. $\epsilon \rightarrow(0, \varepsilon)$, so that the transverse condition $\epsilon \cdot P=0$ now imposes $\varepsilon \cdot p=0$. In the QCD case we note that the Dirac equation now becomes

$$
\begin{aligned}
& \left(p_{\mu} \Gamma_{d}^{\mu}\right) u=m u \\
& \left(p_{\mu} \Gamma_{d}^{\mu}\right) v=-m v,
\end{aligned}
$$

where we have used

$$
\sigma_{D}=\left(\mathbb{I}, \Gamma_{d}\right), \quad \bar{\sigma}_{D}=\left(-\mathbb{I}, \Gamma_{d}\right),
$$

in the chiral representation. Denoting by $W$ and $W^{*}$ the Proca fields obtained from the photons, the construction (2.5) now reads

$$
A_{n}^{\frac{1}{2} \otimes \frac{1}{2}}\left(W_{1} H_{3} \cdots H_{n} W_{2}^{*}\right)=\sum_{\alpha \beta} \frac{K_{\alpha \beta}}{2^{\lfloor d / 2\rfloor-1}} \operatorname{Tr}\left(A_{n, \alpha}^{\mathrm{QCD}}(g \cdots g)\left(\not p_{1}-m\right) \ddagger_{1} \bar{A}_{n, \beta}^{\mathrm{QCD}}(g \cdots g)\left(\not p_{2}-m\right) \ddagger_{2}\right),
$$

where the normalization factor follows from the Dirac trace $\operatorname{tr}(\mathbb{I})=2^{\lfloor D / 2\rfloor}$. Even though our derivation used that $d=2 m-1$ for the reduction procedure, our final result is written explicitly in terms of $d$-dimensional Dirac matrices so we assume it to be valid in generic dimensions. To confirm this we will indeed compute both sides of (2.11) from genericdimensional Lagrangians and find a precise agreement.

From now on we refer to the double-copy theory as the $\frac{1}{2} \otimes \frac{1}{2}$ theory because it is constructed from two (conjugated) copies of massive QCD. As in the massless case, the role of the projectors $\not p_{i} \pm m$ is to put the QCD amplitudes on the support of the massive Dirac equation. With a slight abuse of notation, we have left here the symbol $K_{\alpha \beta}$ for the massive KLT kernel, this simply corresponds to the inverse of the biadjoint amplitude involving two massive scalars of the same species, $K_{\alpha \beta}=m_{n}^{-1}(\alpha \mid \beta)$, see e.g. [86] for details on this theory.

We have thus derived an explicit KLT relation for massive amplitudes of one matter line, (2.11) as a direct consequence of the massless counterpart. The resulting theory will be extended to two matter lines in section 4. The partial amplitudes $A_{n, \alpha}^{\mathrm{QCD}}$ are associated to Dirac spinors in general dimension, as opposed to Majorana ones, and hence the resulting spin-1 field is a complex ${ }^{6}$ Proca state coupled to gravity. Moreover, it follows from the massless case that when all the gravitational states $H_{i}$ are chosen as gravitons, the dilaton and axion field decouple and the theory simply corresponds to Einstein-Hilbert gravity plus a covariantized (minimally coupled) spin-1 Lagrangian. We will see that this holds quite generally and is consistent with the observations made around eq. (1.1) for generic spin.

\footnotetext{
${ }^{6}$ We thank Henrik Johansson for emphasizing this. On the other hand, for the double-copy theories obtained in this work we will drop the distinction between real/complex bosonic matter fields.
} 
In our formula the states $H_{i}$ denote the fat gravitons (2.1) characteristic of the double copy construction. However, a particular feature arises in that amplitudes with an odd number of axion fields vanish. This can be traced back to the symmetry in the two QCD factors of the $\frac{1}{2} \otimes \frac{1}{2}$ construction. To see this, let us slightly rewrite (2.11) as

$$
A_{n}^{\frac{1}{2} \otimes \frac{1}{2}}\left(W_{1} H_{1}^{\mu_{1} \nu_{1}} \cdots H_{n-2}^{\mu_{n-2} \nu_{n-2}} W_{2}^{*}\right)=\sum_{\alpha \beta} K_{\alpha \beta}\left(A_{n, \alpha}^{\mathrm{QCD}}\right)^{\mu_{1} \cdots \mu_{n-2}} \otimes\left(A_{n, \beta}^{\mathrm{QCD}}\right)^{\nu_{1} \cdots \nu_{n-2}},
$$

where

$$
X \otimes Y=\frac{1}{2^{\lfloor d / 2\rfloor-1}} \operatorname{Tr}\left(X\left(\not p_{1}-m\right) \ddagger_{1} \bar{Y}\left(\not p_{2}-m\right) \ddagger_{2}\right) .
$$

It is not hard to check that (see for instance the explicit form in (2.1))

$$
\left(A_{n, \alpha}^{\mathrm{QCD}}\right)^{\mu_{1} \cdots \mu_{n-2}} \otimes\left(A_{n, \beta}^{\mathrm{QCD}}\right)^{\nu_{1} \cdots \nu_{n-2}}=\left(A_{n, \beta}^{\mathrm{QCD}}\right)^{\nu_{1} \cdots \nu_{n-2}} \otimes\left(A_{n, \alpha}^{\mathrm{QCD}}\right)^{\mu_{1} \cdots \mu_{n-2}} .
$$

Now, since the Kernel $K_{\alpha \beta}$ in (2.12) can be arranged to be symmetric in $\alpha \leftrightarrow \beta$, this implies that the r.h.s. of (2.12) is symmetric under the exchange of all $\mu_{i} \leftrightarrow \nu_{i}$ at the same time, namely $\left(\mu_{1}, \mu_{2} \ldots\right) \leftrightarrow\left(\nu_{1}, \nu_{2} \ldots\right)$. However, if we antisymmetrize an odd number of pairs $\left\{\mu_{k}, \nu_{k}\right\}$, i.e. compute the amplitude for an odd number of axions, and symmetrize the rest of the pairs, we obtain an expression which is antisymmetric under the full exchange $\left(\mu_{1}, \mu_{2} \ldots\right) \leftrightarrow\left(\nu_{1}, \nu_{2} \ldots\right)$. Hence amplitudes with an odd number of axions must vanish.

The above considerations imply that the axion field is pair-produced and cannot be sourced by the Proca field. This is also true for amplitudes with no matter (i.e. the massless double copy) and even for amplitudes with more matter lines: for e.g. two matter lines, provided a double copy formula as in section 4, we can test axion propagation by examining all possible factorization channels. Since the factorization always contains amplitudes with either one or none matter lines we conclude that the axion will not emerge in the cut unless introduced also as an external state. The argument carries over for an arbitrary number of matter lines.

The previous fact is surprising from the gravitational perspective since it is known that the axion couples naturally to the spin of matter particles. We interpret this fact as an avatar of the spin- $\frac{1}{2}$ origin of the construction. In appendices A and B we will specialize the construction to $d=4$ : in particular we will show that being a pseudoscalar, the axion can only be sourced when the Proca field decays into a massive pseudoscalar as well, as considered very recently in [79]. In the massless theory such field is obtained by selecting anticorrelated fermion helicities in the r.h.s. of (2.1) which leads to massless (pseudo)scalars instead of photons $\gamma^{ \pm}$[34]. The analysis becomes more involved in higher dimensions. For our purposes here we can neglect these processes and simply keep the theory containing a Proca field, a graviton and a dilaton as a consistent tree-level truncation of the spectrum in arbitrary number of dimensions.

A further clarification is needed regarding the compactification and the dilaton states. In the massless case these are obtained via the replacement

$$
\epsilon_{i}^{\bar{\mu}} \tilde{\epsilon}_{i}^{\bar{\nu}} \rightarrow \frac{\eta^{\bar{\mu} \bar{\nu}}}{\sqrt{D-2}}
$$


where we have denoted the indices as $\bar{\mu}, \bar{\nu}$ to emphasize that the trace is taken in $D=d+1$ dimensions. However, after dimensional reduction we have $\epsilon^{\bar{\mu}} \rightarrow \epsilon^{\mu}$, and we extract the corresponding dilaton via

$$
\epsilon_{i}^{\mu} \tilde{\epsilon}_{i}^{\nu} \rightarrow \frac{\eta^{\mu \nu}}{\sqrt{d-2}}
$$

This means that taking the dimensional reduction does not commute with extracting dilaton states, as e.g. terms of the form $P_{1} \cdot \epsilon P_{2} \cdot \tilde{\epsilon}$ are projected to $P_{1} \cdot P_{2}=p_{1} \cdot p_{2}+m^{2}$ in the first case and to $p_{1} \cdot p_{2}$ in the second case. In order to match certain results in the literature (e.g. [22]) we find that we need to adopt the second construction: first implement dimensional reduction on the fat states, and then project onto either dilatons or gravitons.

Let us close this subsection by providing some key examples of this procedure for $n=3$, 4. The 3 -pt. dilaton amplitude from (2.11), using (2.1), gives

$$
\begin{aligned}
A_{3}^{\frac{1}{2} \otimes \frac{1}{2}}\left(W_{1} \phi W_{2}^{*}\right) & =\frac{2 K_{3}}{2^{\lfloor d / 2\rfloor} \sqrt{d-2}} \operatorname{Tr}\left(A_{3}^{\mu} \phi_{1}\left(\not p_{1}-m\right) \bar{A}_{3 \mu} \oiint_{2}\left(\not p_{2}-m\right)\right), \\
& =\frac{\kappa}{2 \sqrt{d-2}}\left(2 m^{2} \varepsilon_{1} \cdot \varepsilon_{2}+(d-4) k_{3} \cdot \varepsilon_{1} k_{3} \cdot \varepsilon_{2}\right),
\end{aligned}
$$

where we have use the momentum conservation $p_{1}+p_{2}+k_{3}=0$, and the dilaton projection $\epsilon_{3}^{\mu} \tilde{\epsilon}_{3}^{\nu} \rightarrow \frac{\eta^{\mu \nu}}{\sqrt{d-2}}$. This example will exhibit one of the main differences between the $\frac{1}{2} \otimes \frac{1}{2}$ construction and the other cases, namely that the dilaton (and the axion) fields couple differently to matter in each case, as opposed to gravitons which couple universally.

Now we can move on to $n=4$. The only independent QCD amplitude reads

$$
A_{4,1324}^{\mu_{3} \mu_{4}}=-\frac{1}{4} \frac{\gamma^{\mu_{4}}\left(\not p_{1}+\not k_{3}-m\right) \gamma^{\mu_{3}}}{\left(p_{1}+k_{3}\right)^{2}-m^{2}}-\frac{1}{4} \frac{\gamma^{\mu_{3}}\left(\not p_{1}+\not k_{4}-m\right) \gamma^{\mu_{4}}}{\left(p_{1}+k_{4}\right)^{2}-m^{2}}
$$

Analogously,

$$
\bar{A}_{4,1324}^{\mu_{3} \mu_{4}}=-\frac{1}{4} \frac{\gamma^{\mu_{3}}\left(\not p_{1}+\not k_{3}+m\right) \gamma^{\mu_{4}}}{\left(p_{1}+k_{3}\right)^{2}-m^{2}}-\frac{1}{4} \frac{\gamma^{\mu_{4}}\left(\not p_{1}+\not k_{4}+m\right) \gamma^{\mu_{3}}}{\left(p_{1}+k_{4}\right)^{2}-m^{2}}
$$

where the conjugated amplitude is obtained by inverting the direction of the massive line. Note that this ordering corresponds to the QED amplitude.

The full Compton amplitude for fat gravitons can be computed from the double copy $(2.11)$

$$
A_{4}^{\frac{1}{2} \otimes \frac{1}{2}}\left(W_{1} H_{3}^{\mu_{3} \nu_{3}} H_{4}^{\mu_{4} \nu_{4}} W_{2}^{*}\right)=\frac{1}{2^{\lfloor d / 2\rfloor-1}} K_{1324,1324} \operatorname{tr}\left[A_{4}^{\mu_{3} \mu_{4}} \notin_{1}\left(\not p_{1}+m\right) \bar{A}_{4}^{\nu_{3} \nu_{4}} \notin_{2}\left(\not p_{2}+m\right)\right],
$$

where the massive KLT kernel takes the compact form

$$
K_{1324,1324}=\frac{2 p_{1} \cdot k_{3} p_{1} \cdot k_{4}}{k_{3} \cdot k_{4}} .
$$

For instance, the two-dilaton emission amplitude reads

$$
\begin{aligned}
A_{4}^{\frac{1}{2} \otimes \frac{1}{2}}\left(W_{1} \phi_{3} \phi_{4} W_{2}^{*}\right)= & \frac{\kappa^{2} \varepsilon_{1, \alpha} \varepsilon_{2, \beta}^{*}}{32(d-2) p_{1} \cdot k_{3} p_{1} \cdot k_{4} k_{3} \cdot k_{4}}\left\{\left[(d-4)^{2} s_{34}^{2}-16(d-2) p_{1} \cdot k_{3} p_{2} \cdot k_{3}\right]\right. \\
& \times\left[p_{1} \cdot k_{3} k_{4}^{\alpha} k_{3}^{\beta}+p_{2} \cdot k_{3}\left(k_{3}^{\alpha} k_{4}^{\beta}+p_{1} \cdot k_{3} \eta^{\alpha \beta}\right)\right]+2 m^{2} s_{34}\left[4 p _ { 1 } \cdot k _ { 3 } \left(k_{4}^{\alpha} k_{3}^{\beta}-k_{3}^{\alpha} k_{4}^{\beta}\right.\right. \\
& \left.\left.\left.+2 p_{2} \cdot k_{3} \eta^{\alpha \beta}\right)+s_{34}\left((d-4)\left(k_{3}^{\alpha} k_{3}^{\beta}+k_{4}^{\alpha} k_{4}^{\beta}\right)-2\left(k_{3}^{\alpha} k_{4}^{\beta}+m^{2} \eta^{\alpha \beta}\right)\right)\right]\right\},
\end{aligned}
$$


which again exhibits explicit mass dependence in accord with our discussion. On the other hand, extracting the pure graviton emission from (2.1) gives

$$
\begin{aligned}
A_{4}^{\frac{1}{2} \otimes \frac{1}{2}}\left(W_{1} h_{3} h_{4} W_{2}^{*}\right)= & \frac{\kappa^{2} \varepsilon_{1, \alpha} \varepsilon_{2, \beta}^{*}}{2 p_{1} \cdot k_{3} p_{1} \cdot k_{4} k_{3} \cdot k_{4}} p_{1} \cdot F_{3} \cdot F_{4} \cdot p_{1}\left[p_{1} \cdot p_{3} F_{4}^{\mu \alpha} F_{3, \mu}^{\beta}\right. \\
& \left.+p_{1} \cdot k_{4} F_{3}^{\mu \alpha} F_{4, \mu}^{\beta}+F_{3}^{\alpha \beta} p_{1} \cdot F_{4} \cdot p_{2}+F_{4}^{\alpha \beta} p_{1} \cdot F_{3} \cdot p_{2}+p_{1} \cdot F_{3} \cdot F_{4} \cdot p_{1} \eta^{\alpha \beta}\right],
\end{aligned}
$$

where $F_{i}^{\mu \nu}=2 k_{i}^{[\mu} \epsilon_{i}^{\nu]}$. Quite non-trivially, we find that the Dirac trace leads to a factorized formula. The underlying reason is of course that the graviton amplitudes are universal as announced. This means these results can also be obtained via the $0 \otimes 1$ factorization that we introduce in the next subsection.

\subsubsection{Exempli gratia: the multipole expansion}

We have introduced the operation (2.11) with a slight modification in [47]. This is because the main utility of this construction is not the fact that we can build gravitational amplitudes by squaring those of QCD (we have just seen that the former follow from a dimensional reduction of the Einsten-Maxwell system), but the fact that by rearranging the massive QCD amplitudes in a multipole form we obtain a multipole expansion on the gravitational side [87-93]. To our knowledge there is no systematic way of performing such expansion in general (however, see [94] for a recent discussion).

For spin $\frac{1}{2}$ the multipole expansion is obtained by writing the operator $A_{n}^{\mathrm{QCD}}$ in powers of the intrinsic angular-momentum operator $J^{\mu \nu}=\frac{\gamma^{\mu \nu}}{2}=\frac{1}{4} \gamma^{[\mu} \gamma^{\nu]}$. This is usually achieved by employing the Dirac equation. For instance, at $n=3$ it is easy to derive the textbook identity

$$
\bar{u}_{2} A_{3}^{\mathrm{QCD}} v_{1} \propto m \epsilon_{\mu} \bar{u}_{2} \gamma^{\mu} v_{1}=\epsilon_{3} \cdot p_{1} \bar{u}_{2} v_{1}-\frac{g}{4} k_{3 \mu} \epsilon_{3 \nu} \bar{u}_{2} \gamma^{\mu \nu} v_{1}
$$

which also holds for the operators in (2.11) as they are under the support of the Dirac equation. The first term we call the scalar piece while the second we associate to a dipole $[56,57]$. Here we interpret $g=2$ as the corresponding form factor and its (tree-level) value is fixed for a Dirac spinor coupled to a photon/gluon. In the following sections we shall see that this is not true for higher spins and in fact it is the double copy criteria above what fixes $g=2$ in general $[47,56,57]$.

Now consider two such multipole operators $X, Y$ of order $p, q$ respectively, namely $X \sim\left(\gamma^{\mu \nu}\right)^{p}$ and $Y \sim\left(\gamma^{\mu \nu}\right)^{q}$ acting on Dirac spinors. As they involve an even number of gamma matrices, and the Dirac trace vanishes for an odd number of such, we have

$$
\operatorname{Tr}\left(X(g \cdots g)\left(\not p_{1}-m\right) \ddagger_{1} \bar{Y}(g \cdots g)\left(\not p_{2}-m\right) \ddagger_{2}\right)=\operatorname{Tr}\left(X p_{1} \varepsilon_{1} \bar{Y} p_{2} \varepsilon_{2}\right)+m^{2} \operatorname{Tr}\left(X \varepsilon_{1} \bar{Y} \varepsilon_{2}\right),
$$

where the conjugated operator $\bar{Y}$ is obtained by $\gamma^{\mu \nu} \rightarrow-\gamma^{\mu \nu}$. In the cases studied in [47] (for $n=3,4$ ) both terms in the r.h.s. coincide and hence we defined the double copy product simply as

$$
X \odot Y=\frac{1}{2^{\lfloor D / 2\rfloor}} \operatorname{Tr}\left(X \varepsilon_{1} \bar{Y} \varepsilon_{2}\right),
$$

i.e. using twice the second term. At $s=\frac{1}{2}$ we explicitly tested this definition for operators up to the quadratic order in $\gamma^{\mu \nu}$. Let us here just recall the example of $A_{3}$, which exhibits 
an explicit exponential form. Combining the Dirac algebra with 3-pt. kinematics we find $\left(k_{\mu} \epsilon_{\nu} \gamma^{\mu \nu}\right)^{2}=0$, which we use to rewrite $(2.24)$ as

$$
\bar{u}_{2} A_{3}^{\mathrm{QCD}} v_{1} \propto \epsilon \cdot p_{1} \times \bar{u}_{2} e^{J} v_{1}
$$

where $J$ is a Lorentz generator that reads

$$
J=-\frac{k_{3 \mu} \epsilon_{3 \nu}}{\epsilon_{3} \cdot p_{1}} J^{\mu \nu}=-\frac{k_{3 \mu} \epsilon_{3 \nu}}{\epsilon_{3} \cdot p_{1}} \frac{\gamma^{\mu \nu}}{2} .
$$

The exponential form for $s=\frac{1}{2}$ generators is only linear in this case since higher multipoles vanish. Note now that while the second equality holds for $s=\frac{1}{2}$, the generator $J$ itself makes sense in any representation [47]. In the representation $\left(J^{\mu \nu}\right)_{\beta}^{\alpha}=\eta^{\alpha[\mu} \delta_{\beta}^{\nu]}$ we can check that $\left(e^{J}\right)_{\alpha}^{\beta} p_{1}^{\alpha}=\left(p_{1}+k\right)^{\beta}=-p_{2}^{\beta}$ and hence the generator acts as a boost $p_{1} \rightarrow-p_{2}$. Now we can plug the operator $(2.27)$ and its conjugate in (2.25) and check that in fact both terms yield the same contribution:

$$
\begin{aligned}
A_{3}^{\mathrm{QCD}} \otimes A_{3}^{\mathrm{QCD}} & \propto \operatorname{Tr}\left(e^{J}\left(\not p_{1}-m\right) \ddagger_{1} e^{-J}\left(\not p_{2}-m\right) \ddagger_{2}\right), \\
& =\operatorname{Tr}\left(e^{J} p_{1} e^{-J} e^{J} \varepsilon_{1} e^{-J} p_{2} \varepsilon_{2}\right)+m^{2} \operatorname{Tr}\left(e^{J} \varepsilon_{1} e^{-J} \varepsilon_{2}\right), \\
& =-\operatorname{Tr}\left(p_{2} \tilde{\varepsilon}_{2} p_{2} \varepsilon_{2}\right)+m^{2} \operatorname{Tr}\left(\tilde{\varepsilon}_{2} \varepsilon_{2}\right)=2 m^{2} \operatorname{Tr}(\mathbb{I}) \tilde{\varepsilon}_{2} \cdot \varepsilon_{2},
\end{aligned}
$$

where $\tilde{\varepsilon}_{2}^{\alpha}=\left(e^{J}\right)_{\beta}^{\alpha} \varepsilon_{1}^{\beta}$ is a new polarization state for $p_{2}$, that is, it satisfies $p_{2} \cdot \tilde{\varepsilon}_{2}=0$. Thus we obtain the gravitational (Proca) amplitude as

$$
A_{3}^{\frac{1}{2} \otimes \frac{1}{2}} \propto \epsilon_{3} \cdot p_{1} \times \varepsilon_{2} \cdot e^{J} \cdot \varepsilon_{1}=\epsilon_{3} \cdot p_{1} \varepsilon_{2} \cdot \varepsilon_{1}-k_{3 \mu} \epsilon_{3 \nu} \varepsilon_{2}^{\alpha}\left(J^{\mu \nu}\right)_{\alpha}^{\beta} \varepsilon_{1 \beta},
$$

where higher multipoles also vanish for $s=1$, in contrast with higher spins (see (2.35)). This simple example shows that the exponential form is preserved under double copy (this is particular of $n=3$ ), but more importantly it shows the general fact that, as observed in [47], the gravitational amplitude is obtained in multipole form as well. The multipole operators can be double copied via general rules, and in turn the resulting multipole expansion can be used to decode the classical information contained in the amplitude.

\subsection{General case with $s, \tilde{s} \leq 1$}

Let us now give general considerations regarding the massive KLT construction for $s, \tilde{s} \leq 1$. Following the philosophy of [53] we know massive amplitudes in GR (for $s \leq 2$ ) and QCD (for $s \leq 1$ ) can be adjusted so that they posses a smooth high energy limit, i.e. they are free of $1 / m$ terms. This criteria was used as a definition of minimal coupling [53] in these cases and completely fixed the $n=3$ amplitudes. On the other hand, it is known that for higher spins the situation changes drastically and such divergences cannot be avoided [61, 81] (more recently, see [51]), which reflects the fact that interacting massless higher spins theories are inconsistent $[53,95]$. Here we will exploit the fact that at low spins the minimal coupling amplitudes are " $1 / m$-free" to construct them directly from their massless version via dimensional reduction. We will also see how this criteria interacts with 
the double copy and the natural value of $g$ (as defined in the previous section), making contact with the results of e.g. [51, 56, 57, 60, 96].

From a purely group-theoretical perspective it is direct to construct massive states in general dimensions for spins $s=0, \frac{1}{2}, 1, \frac{3}{2}, 2$ out of products of two lower spins. The cases $s=0=0+0$ and $s=\frac{1}{2}=0+\frac{1}{2}$ are obvious while the cases for $s=1$ we have already introduced. The remaining situations are $2=1+1$ and $\frac{3}{2}=\frac{1}{2}+1$, i.e. ${ }^{7}$

$$
\begin{aligned}
& \varepsilon^{\mu} \tilde{\varepsilon}^{\nu} \rightarrow \phi^{\mu \nu}=\varepsilon^{(\mu} \tilde{\varepsilon}^{\nu)}-\left(\eta^{\mu \nu}-\frac{p^{\mu} p^{\nu}}{m^{2}}\right) \varepsilon \cdot \tilde{\varepsilon}, \\
& \psi^{\alpha} \varepsilon^{\mu} \rightarrow \Psi^{\alpha \mu}=\psi^{\alpha} \varepsilon^{\mu}-\varepsilon^{\nu} \frac{\left(\gamma^{\mu} \gamma_{\nu} \psi\right)^{\alpha}}{d}
\end{aligned}
$$

Our goal is to construct an interacting theory containing only such massive states (e.g. $\phi$ or $\Psi$ ) but no other massive particle, i.e. as consistent truncation of the full double copy. For the case $s=\frac{3}{2}$ we note that we will only consider the product $1+\frac{1}{2}$ and not $0+\frac{3}{2}$. This is because, at the massless level, theories with an interacting gravitino field are well known to be inconsistent unless coupled to GR, and hence the factor $\frac{3}{2}$ in this construction cannot correspond to a QCD theory. ${ }^{8}$ A similar situation holds for the case $0+2$, see e.g. $[81,100,101]$.

Detour: arbitrary spin at $\boldsymbol{n}=\mathbf{3}$. The massless origin of all these constructions should be by now clear. Let us take a brief detour to emphasize some remarkable properties at $n=3$. In $D=4$, the massless three-point amplitude is fixed from helicity weights as [95],

$$
A_{3}^{h_{3}, h} \sim\left(\frac{\langle 13\rangle}{\langle 23\rangle}\right)^{2 h}\left(\frac{\langle 13\rangle\langle 32\rangle}{\langle 12\rangle}\right)^{h_{3}}
$$

for an state of arbitrary $h$ emitting a gluon $\left(h_{3}=1\right)$ or a graviton $\left(h_{3}=2\right)$. Consequently, it directly satisfies the double copy relation

$$
A_{3}^{\mathrm{gr}, h+\bar{h}}=K_{3} A_{3}^{\mathrm{QCD}, h} A_{3}^{\mathrm{QCD}, \bar{h}} .
$$

On the other hand, by implementing the multipole expansion, in [47] we have found that the same relation can be imposed for massive amplitudes of arbitrary spin, and fixes their full form as

$$
A_{3}^{h_{3}, s} \sim\left(\epsilon_{3} \cdot p_{1}\right)^{h_{3}} \varepsilon_{2} \cdot \exp \left(-\frac{k_{3 \mu} \epsilon_{3 \nu}}{\epsilon_{3} \cdot p_{1}} J_{s}^{\mu \nu}\right) \cdot \varepsilon_{1},
$$

where $J_{s}^{\mu \nu}$ is the generator in e.g. (2.28) naturally adapted to higher spin $s .{ }^{9}$ Observe that this form does not depend explicitly on the mass and, as noted in [104], reduces to (2.33)

\footnotetext{
${ }^{7}$ Even though these projections hold in arbitrary dimension, in general for $d>4$ we will have an increased number of states labeled by additional Casimirs of the Lorentz group and not just the spin quantum number [97].

${ }^{8}$ Even though spin- $\frac{3}{2}$ QED can be made free of $1 / m$ divergences [60] as opposed to $s \geq 2$, unitarity and causality inconsistencies (related to the Velo-Zwanziger problem [98]) have been stressed in e.g. [96, 99].

${ }^{9} \mathrm{~A}$ local form of this amplitude can be found in $[47,102,103]$, which however features $1 / m$ divergences.
} 
when written in terms of the $D=4$ spinor helicity variables. ${ }^{10}$ Hence $(2.35)$ is nothing but the natural extension of (2.33) to generic dimension and helicities, whose dimensional reduction in the sense of the previous section is trivial. Curiously, when interpreted as a $D=4$ massless amplitude this object is known to be inconsistent with locality for $|h|>1$ (or analogously $s>1$ ) whereas in the massive case it has the physical interpretation given in $[12,50,51]$. On the other hand, these inconsistencies will only appear in the "four-point test" $[53,95]$, namely by computing $A_{4}^{\mathrm{QCD}}$ or $A_{4}^{\mathrm{gr}}$. In the massive case they can be cured by including contact interactions [51].

Arbitrary multiplicity at low spins. From the above discussion we see that at least at low spins we can extend the relation (2.34) and its compactification to arbitrary multiplicity, since the massless theory is healthy. The starting QCD theories for scalars, Dirac fermions and gluons are standard and catalogued in the next section. Let us then write

$$
A_{n}^{h+\bar{h}}\left(\varphi_{1}^{h+\bar{h}} H_{3} \cdots H_{n} \varphi_{2}^{-h-\bar{h}}\right):=\frac{1}{2} \sum_{\alpha \beta} K_{\alpha \beta} A_{n, \alpha}^{\mathrm{QCD}}\left(\varphi_{1}^{h} g_{3} \cdots g_{n} \varphi_{2}^{-h}\right) A_{n, \beta}^{\mathrm{QCD}}\left(\varphi_{1}^{\bar{h}} g_{3} \cdots g_{n} \varphi_{2}^{-\bar{h}}\right),
$$

where we have denoted by $\varphi_{i}^{h}$ the state of helicity $h$ and particle label $i$. This extends the relation (2.1) for the cases $h, \bar{h} \leq 1$. We can also uplift it to arbitrary dimensions. Following the previous section we first rewrite the amplitudes in terms of the corresponding polarization vectors/spinors and the implement the tensor products $\otimes$ between representations (besides the trivial cases, these are just the massless versions of (2.31)). For simplicity of the argument we regard (2.36) as a definition of the object $A_{n}^{\mathrm{gr}}$, and we claim that it corresponds to a tree-level amplitude in a certain QFT coupled to gravity. We recall from the previous section that this is because 1) diffeomorphism (gauge) invariance and crossingsymmetry are manifest and 2) tree-level unitarity follows from general arguments $[3,85]$. This means that we just need to construct a corresponding Lagrangian to identify the theory, which we will do for most cases in section 3 .

We have already explained how under the dimensional reduction $D=d+1 \rightarrow d$ we obtain massive momenta and the corresponding propagators. We have also shown how the $D$-dimensional polarization vectors/spinors of the compactified particles, $\varepsilon^{\mu}$ and $u^{\alpha}$, can now be regarded as satisfying the corresponding massive wave equations. The result

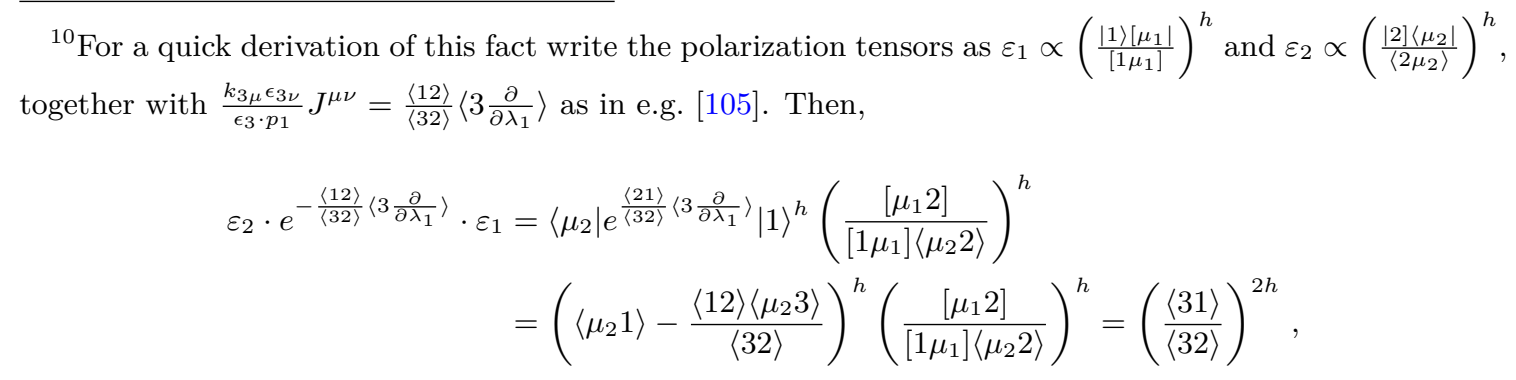

where we have used that $e^{-\frac{\langle 12\rangle}{\langle 32\rangle}\left\langle 3 \frac{\partial}{\partial \lambda_{1}}\right\rangle}$ acts as a Lorentz boost on $|1\rangle$, see appendix B in [47]. Finally, the $h_{3}$ dependence is also the same in (2.33) and (2.35). 
of (2.36) after this procedure leads to the general formula for one-massive line

$$
A_{n}^{s+\tilde{s}}\left(\varphi_{1}^{s+\tilde{s}} H_{3} \cdots H_{n} \varphi_{2}^{s+\tilde{s}}\right):=\frac{1}{2} \sum_{\alpha \beta} K_{\alpha \beta} A_{n, \alpha}^{\mathrm{QCD}}\left(\varphi_{1}^{s} g_{3} \cdots g_{n} \varphi_{2}^{s}\right) \otimes A_{n, \beta}^{\mathrm{QCD}}\left(\varphi_{1}^{\tilde{s}} g_{3} \cdots g_{n} \varphi_{2}^{\tilde{s}}\right)
$$

which holds for $s, \tilde{s} \leq 1$ and has a smooth high-energy limit by construction. Thus, this gives a double-copy formula for the minimally-coupled partial amplitudes defined in the sense of [53].

Even though we have not yet specified the theory, let us momentarily restrict the states $H_{i}$ to gravitons. We have explicitly checked, by inserting massive spinor-helicity variables, that in $D=4$ we can obtain the gravitational and QCD amplitudes given in [53] for $n=3,4$, see (2.46) below. This establishes a $D=4$ double-copy formula between these amplitudes, analogous to the one studied in appendix A. In general dimensions, we have also checked that this agrees with the amplitudes and double copy for $s=0, \tilde{s} \neq 0$ pointed out in [58]. More generally we can use (2.31) to recover the $1 \otimes 1$ construction studied by us in [47] or extend it with the case $1 \otimes \frac{1}{2}$. We remark that these are precisely the gravitational amplitudes used to obtain perturbative black hole observables in [50, 52, 104, 106, 107], and that for the all-graviton case the l.h.s. of (2.37) is unique given the sum $s+\tilde{s}$.

We now provide simple examples to illustrate these points. In the rest of this section we shall indistinctly use $\varepsilon_{2}$ or $\varepsilon_{2}^{*}$ to refer to the (conjugated) polarization of the outgoing massive state.

\subsubsection{Non-universality of dilaton couplings}

As opposed to gravitons, we have anticipated that the dilaton field couples differently in the $0 \otimes 1$ than in the $\frac{1}{2} \otimes \frac{1}{2}$ case. So let us compute the amplitude $A_{3}\left(W_{1} \phi W_{2}^{*}\right)$ via double copy of $s=0$ and $s=1$. This is to say, we take the trace of

$$
A_{3}^{0 \otimes 1}\left(W_{1} H^{\mu \nu} W_{2}^{*}\right)=A_{3}^{\mathrm{QCD}, s=0}\left(\varphi_{1} g^{\mu} \varphi_{2}\right) A_{3}^{\mathrm{QCD}, s=1}\left(W_{1} g^{\nu} W_{2}^{*}\right)
$$

i.e. the $0 \otimes 1$ double copy, and contrast it with (2.17) from the $\frac{1}{2} \otimes \frac{1}{2}$ double copy. The spin1 QCD factor arising from dimensional reduction is equivalent to a covariantized Proca action plus a correction on the gyromagnetic ratio $g$, see next section. Explicitly,

$$
A_{3}^{\mathrm{QCD}, s=1}\left(W_{1} g^{\mu} W_{2}^{*}\right)=p_{1}^{\mu} \varepsilon_{1} \cdot \varepsilon_{2}-\varepsilon_{1}^{\alpha}\left(J^{\mu \nu}\right)_{\alpha}^{\beta} \varepsilon_{2 \beta} k_{3 \nu}
$$

where we used that $\left(J^{\mu \nu}\right)_{\beta}^{\alpha}=\eta^{\alpha[\mu} \delta_{\beta}^{\nu]}$ according to our conventions in (2.30). Recalling that for spin- $0 A_{3}^{\mu} \propto p_{1}^{\mu}$, the trace of (2.38) gives

$$
A_{3}^{0 \otimes 1}\left(W_{1} \phi W_{2}^{*}\right)=\frac{\kappa}{\sqrt{d-2}}\left(m^{2} \varepsilon_{1} \cdot \varepsilon_{2}+k_{3} \cdot \varepsilon_{1} k_{3} \cdot \varepsilon_{2}\right),
$$

where we restored the coupling $\kappa$ in order to be more precise. We now observe that this differs from (2.17) in a term proportional to $\varepsilon_{1} \cdot k_{3} \varepsilon_{2} \cdot k_{3}$, controlled by a coupling $\phi F^{2}$ with the matter field that we derive in the next section. At first this may look like a contradiction given that we pinpointed the massless origin of this double copy, namely eq. (2.34). Here $A_{3}\left(W_{1} \phi W_{2}^{*}\right)$ should be uniquely fixed by little-group as happened for the 
graviton case (2.35). The difference however lies in the coupling constant, which vanishes in the $d \rightarrow 4, m \rightarrow 0$ limit for $A_{3}^{\frac{1}{2} \otimes \frac{1}{2}}\left(W_{1} \phi W_{2}^{*}\right)$ but not for $A_{3}^{0 \otimes 1}\left(W_{1} \phi W_{2}^{*}\right)$. Hence the reason why graviton amplitudes are the same in both $\frac{1}{2} \otimes \frac{1}{2}$ and $0 \otimes 1$ double-copies is not only because of its massless form (2.33), but also because the coupling $\kappa$ is fixed by the equivalence principle.

A final and crucial remark is as follows. From general considerations it is known that the dilaton cannot couple linearly to the spin of a matter line [26, 27]. This is consistent, as we will see that (2.40) contains only a quadrupole $\sim J^{2}$ term, but appears in contradiction with the fact that $A^{s=1}$ in (2.39), which carries the spin dependence, seems to have a dipole and no quadrupole. The resolution of this puzzle comes from distinguishing two types of multipoles. The first type are the covariant multipoles carrying the action of the full Lorentz group $\mathrm{SO}(d-1,1)$, as generated by $J^{\mu \nu}$. The second type are the rotation multipoles defined by the condition $p_{\mu} S^{\mu \nu}=0$ with respect to e.g. the average momentum $p=\frac{p_{1}+p_{2}}{2}$. They generate the $\mathrm{SO}(d-1)$ rotation subgroup and in the classical limit represent the classical spin-tensor of compact objects. The relation between the two multipoles is the decomposition $\mathrm{SO}(d-1,1) \rightarrow \mathrm{SO}(d-1)$ explained in appendix A of [47] (see also [107]), such that one can write $J_{\mu \nu}=S_{\mu \nu}+$ boost terms. Using this, (2.39) can be written as

$$
A_{3}^{\mathrm{QCD}, s=1, \mu}=p^{\mu}\left(1+\frac{k_{3 \mu} S^{\mu \alpha} S_{\alpha}^{\nu} k_{3 \nu}}{m^{2}(d-3)}\right)-S^{\mu \nu} k_{3 \nu},
$$

where the quadrupole term $S^{\mu \alpha} S_{\alpha}^{\nu}$ is obtained precisely from the boost piece and we have stripped polarization states. ${ }^{11}$ The double copy now gives

$$
\begin{aligned}
A_{3}^{0 \otimes 1}\left(W_{1} \phi W_{2}^{*}\right) & =\frac{\kappa}{\sqrt{d-2}} p_{\mu}\left[p^{\mu}\left(1+\frac{k_{3 \mu} S^{\mu \alpha} S_{\alpha}^{\nu} k_{3 \nu}}{m^{2}(d-3)}\right)-S^{\mu \nu} k_{3 \nu}\right] \\
& =\frac{\kappa m^{2}}{\sqrt{d-2}}\left(1+\frac{k_{3 \mu} S^{\mu \alpha} S_{\alpha}^{\nu} k_{3 \nu}}{m^{2}(d-3)}\right) .
\end{aligned}
$$

Comparing this to our previous result, it is clear that the term $k_{3} \cdot \varepsilon_{1} k_{3} \cdot \varepsilon_{2}$ in $(2.40)$ is in direct correspondence with the quadrupole operator. A similar argument holds for the $\frac{1}{2} \otimes \frac{1}{2}$ theory: in this case there is genuinely no quadrupole contribution in the QCD factor,

$$
A_{3}^{\mathrm{QCD}, s=\frac{1}{2}, \mu}=p^{\mu}-S^{\mu \nu} k_{3 \nu},
$$

whereas in the double copy $A^{\frac{1}{2} \otimes \frac{1}{2}}$ the linear-in-spin terms again cancel due to $p_{\mu} S^{\mu \nu}=0$. We are left again with a quadrupole term $\sim S^{2}$, as can be also seen from (2.17). We conclude that the $\frac{1}{2} \otimes \frac{1}{2}$ and $0 \otimes 1$ theories differ in the dilaton coupling only at the level of the matter quadrupole. We come back to this point during section 4.3 in the context of classical double copy.

\subsubsection{Compton amplitude and the $g$ factor}

Moving on to $n=4$, we can explore the interplay between the double copy and the multipole expansion. Let us first quote here the spin-1 QCD result for general gyromagnetic factor

\footnotetext{
${ }^{11}$ Here the massive polarization vectors have been removed and the quantum amplitude is understood to be an operator acting on them. On the other hand, in the classical context, $S^{\mu \nu}$ is interpreted as a spin tensor (c-number) describing the intrinsic rotation of the classical object.
} 
$g$ computed by Holstein in [56]

$$
\begin{aligned}
A_{4}^{\mathrm{QCD}, s=1}(1324)= & \frac{1}{4}\left\{-2 \varepsilon_{1} \cdot \varepsilon_{2}\left[\frac{\epsilon_{3} \cdot p_{1} \epsilon_{4} \cdot p_{2}}{p_{1} \cdot k_{3}}+\frac{\epsilon_{3} \cdot p_{2} \epsilon_{4} \cdot p_{1}}{p_{1} \cdot k_{4}}+\epsilon_{3} \cdot \epsilon_{4}\right]\right. \\
& -g\left[\varepsilon_{1} \cdot F_{4} \cdot \varepsilon_{2}\left(\frac{\epsilon_{3} \cdot p_{1}}{p_{1} \cdot k_{3}}-\frac{\epsilon_{3} \cdot p_{2}}{p_{1} \cdot k_{4}}\right)+\varepsilon_{1} \cdot F_{3} \cdot \varepsilon_{2}\left(\frac{\epsilon_{4} \cdot p_{2}}{p_{1} \cdot k_{3}}-\frac{\epsilon_{4} \cdot p_{1}}{p_{1} \cdot k_{4}}\right)\right] \\
& +g^{2}\left[\frac{1}{2 p_{1} \cdot k_{3}} \varepsilon_{1} \cdot F_{3} \cdot F_{4} \cdot \varepsilon_{2}-\frac{1}{2 p_{1} \cdot k_{4}} \varepsilon_{1} \cdot F_{4} \cdot F_{3} \cdot \varepsilon_{2}\right] \\
& -\frac{(g-2)^{2}}{m^{2}}\left[\frac{1}{2 p_{1} \cdot k_{3}} \varepsilon_{1} \cdot F_{3} \cdot p_{1} \varepsilon_{2} \cdot F_{4} \cdot p_{2}\right. \\
& \left.\left.-\frac{1}{2 p_{1} \cdot k_{4}} \varepsilon_{1} \cdot F_{4} \cdot p_{1} \varepsilon_{2} \cdot F_{3} \cdot p_{1}\right]\right\},
\end{aligned}
$$

where $F_{i}^{\mu \nu}=2 k_{i}^{[\mu} \epsilon_{i}^{\nu]}$. Here all momenta are outgoing and satisfy the on-shell conditions $p_{1}^{2}=p_{2}^{2}=m^{2}$ and $k_{3}^{2}=k_{4}^{2}=0$. The covariantized Proca theory is obtained by setting $g=1$ and hence contains a $1 / m$ divergence. On the other hand, if the Proca field is identified with a $W^{ \pm}$boson of the electroweak model we obtain $g=2$ and completely cancel the $1 / m$ term. This is a general feature of the $g=2$ theory at any multiplicity [60]. Moreover, in this case we observe not only a well behaved high energy limit, but also not apparent dependence on $m$ at all! This means that the amplitude is essentially equal to its massless limit, which corresponds to a $n=4$ color-ordered gluon amplitude, see section 2.2.3 below.

From the above we find that for this amplitude setting $g=2$ will automatically yield to the double copy relation (2.37). This is the underlying reason for the result found in $[56,57]$ for the natural value of $g$. The converse is also true as gravitational amplitudes always have $g=2$, thus imposing the same value on its QCD factors. The universality of $g$ is a feature of the gravitational Lagrangians, independently of the covariantization or the couplings considered. It was checked explicitly in [51] and is a direct consequence of the universal subleading soft theorem in gravity [47]. This contrasts to QCD in that only the leading soft factor is universal there and hence $g$ becomes a parameter. Finally, it can also be understood from the fact that both rotating black hole or neutron stars also yield $g=2$ indistinctly in classical GR [96].

Let us elaborate on the relation between (2.44) and the 4-gluon amplitude. Pretend that (2.44) (with $g=2$ ) is indeed the massless amplitude. As we compactify we must send $p_{i} \rightarrow P_{i}=\left(p_{i}, \pm m\right)$ and $k_{i} \rightarrow\left(k_{i}, 0\right)$, while setting the polarizations $\varepsilon_{i}, \epsilon_{i}$ to lie also in $D-1$ dimensions. As the amplitude itself only depends on $p_{i}$ through $P_{i} \cdot k_{j}$ and $P_{i} \cdot \epsilon_{j}$ the extra dimensional component of $P_{i}$ drops and the mass $m$ simply does not appear. More generally, the reader can convince themselves that the only appearances of $m$ are through 1) $P_{1} \cdot P_{2}=p_{1} \cdot p_{2}+m^{2}$ or 2) $P_{1} \cdot \epsilon_{i} P_{2} \cdot \epsilon_{j}$, which we have seen lead to $p_{1} \cdot p_{2}$ after dilaton projection. In the first case we can use momentum conservation to write $P_{1} \cdot P_{2}=\sum_{i<j} k_{i} \cdot k_{j}$ and effectively cancel the mass dependence. Hence, if we choose a basis of kinematic invariants that excludes $P_{1} \cdot P_{2}$ the compactification will be trivial: the amplitudes $A_{n}$ will essentially be identical to their massless limit except in the cases of dilaton amplitudes, since they contain terms like $p_{1} \cdot p_{2}=-m^{2}+\sum_{i<j} k_{i} \cdot k_{j}$. The same 
observation applies to the KLT construction (2.37) and the KLT kernel introduced in the previous section. We will extend these observations to more matter lines in section 4 .

Note also that the explicit mass dependence can as well be hidden by means of $d=4$ massive spinor-helicity variables. ${ }^{12}$ For instance, using these variables eq. (2.44) with $g=2$ reads

$$
A_{4}^{\mathrm{QCD}, s=1}(1324) \propto \frac{\langle 3|1| 4]^{2}}{p_{1} \cdot k_{3} p_{1} \cdot k_{4}}\left(\left[1^{a} 3\right]\left\langle 42^{b}\right\rangle+\left\langle 1^{a} 4\right\rangle\left[2^{b} 3\right]\right)^{2}
$$

In this form the double copy can be performed as in appendix A. For instance, from two copies of the previous spin- 1 amplitude we obtain the following spin- 2 amplitude:

$$
A_{4}^{\mathrm{gr}, s=2} \propto \frac{\langle 3|1| 4]^{4}}{p_{1} \cdot k_{3} p_{1} \cdot k_{4} k_{3} \cdot k_{4}}\left(\left[1^{a} 3\right]\left\langle 42^{b}\right\rangle+\left\langle 1^{a} 4\right\rangle\left[2^{b} 3\right]\right)^{4}
$$

This result has been used to construct observables associated to the Kerr Black-Hole in [50, 51]. Here we can conclude that such amplitude is nothing but the 4-graviton amplitude in higher dimensions. Again, since there are no massless higher spin particles in flat space, this framework provides a natural explanation for the fact that $A_{4}^{\mathrm{gr}, s>2}$ and $A_{4}^{\mathrm{QCD}, s>1}$ must contain $\frac{1}{m}$ divergences.

\subsubsection{Universality of scalar multipole}

We close this section with a final observation on the multipole expansion. In [47] we observed that the multipoles of section 2.1.1 were universal with respect to spin for gluon or graviton emission. This means for instance that we can consider $A_{4}^{\mathrm{QCD}, s}$ and the multipole decomposition should be the same for $s=0$ and $s=1$. Now we can prove this explicitly for the scalar multipole, which is by definition the term proportional to the zeroth power of the angular momentum $J^{\mu \nu}$. In (2.44) that would correspond to $\varepsilon_{1} \cdot\left(J^{\mu \nu}\right)^{0} \cdot \varepsilon_{2}=\varepsilon_{1} \cdot \varepsilon_{2}$, e.g.

$$
\left.A_{4}^{\mathrm{QCD}, s=1}\right|_{J^{0}}=\frac{1}{2}\left(\frac{\epsilon_{3} \cdot p_{1} \epsilon_{4} \cdot p_{2}}{p_{1} \cdot k_{3}}-\frac{\epsilon_{3} \cdot p_{2} \epsilon_{4} \cdot p_{1}}{p_{1} \cdot k_{4}}-\epsilon_{3} \cdot \epsilon_{4}\right) .
$$

On the other hand for the spin-0 representation the multipole expansion is trivial $J^{\mu \nu} \rightarrow 1$. Thus the claim of universality becomes, at any multiplicity,

$$
\left.A_{n}^{\mathrm{QCD}, s=1}\right|_{J^{0}}=A_{n}^{\mathrm{QCD}, s=0} .
$$

But this relation is now obvious from the massless perspective. In fact the amplitude $A_{4}^{\mathrm{QCD}, s=0}$ in the massless limit is simply the special Yang-Mills Scalar (YMS) theory considered in e.g. $[69,108]$ and reviewed in next section. It is known that amplitudes with two scalars in such theory can be obtained from the pure Yang-Mills amplitude by allowing two polarization vectors to explore a one-dimensional internal space (see [109] for a review). More specifically we obtain scalars by setting $\varepsilon_{i}=(0, \ldots, 0,1)$ while the remaining polarizations and momenta are written as $\epsilon_{i} \rightarrow\left(\epsilon_{i}, 0\right)$ and $p_{i} \rightarrow\left(p_{i}, 0\right)$. Thus the only surviving contraction involving $\varepsilon_{1}$, $\varepsilon_{2}$ is precisely $\varepsilon_{1} \cdot \varepsilon_{2}$. Hence at any multiplicity the YMS amplitude is the coefficient of $\varepsilon_{1} \cdot \varepsilon_{2}$ in the pure gluon amplitude and (2.48) follows.

\footnotetext{
${ }^{12}$ See [53] for the details on this formalism and [47, 50] for a construction of these amplitudes via soft factors.
} 
In the case of the gravitational theory the same situation arises for the case the fat states $H_{i}$ are only gravitons $h_{i}$. As explained in section 2.2.1, these amplitudes should depend on $s+\tilde{s}$ and not on $s, \tilde{s}$ individually. In particular for $s+\tilde{s} \leq 1$ they read

$$
A_{n}^{0 \otimes s+\tilde{s}}\left(\varphi_{1}^{s+\tilde{s}} h_{3} \cdots h_{n} \varphi_{2}^{s+\tilde{s}}\right):=\frac{1}{2} \sum_{\alpha \beta} K_{\alpha \beta} A_{n, \alpha}^{\mathrm{QCD}}\left(\varphi_{1}^{0} \cdots \varphi_{2}^{0}\right) A_{n, \beta}^{\mathrm{QCD}}\left(\varphi_{1}^{s+\tilde{s}} \cdots \varphi_{2}^{s+\tilde{s}}\right)
$$

i.e. the whole spin dependence can be put in a single QCD factor. Applying the construction of the previous paragraph to such factor we find

$$
\left.A_{n}^{\mathrm{gr}, s}\right|_{J^{0}}=A_{n}^{\mathrm{gr}, 0}
$$

for graviton emission. Observe that in the case of dilaton fields this relation breaks down: first, the 1.h.s. of (2.49) depends not only on $s+\tilde{s}$ but on $s, \tilde{s}$ individually. Second, for e.g. spin-1 terms of the type $\varepsilon_{1} \cdot \epsilon_{i} \varepsilon_{2} \cdot \epsilon_{j} \tilde{\epsilon}_{i} \cdot \tilde{\epsilon}_{j}$ in $A_{n, \beta}^{\mathrm{QCD}}$ will lead to extra pieces proportional to $\varepsilon_{1} \cdot \varepsilon_{2}$ in $A_{n, \beta}^{\mathrm{gr}}$, thereby altering its scalar piece. It would be interesting to generalize these arguments for higher multipoles $\left(J^{\mu \nu}\right)^{n}$.

\section{Constructing the Lagrangians}

In this section we will provide the Lagrangians associated to the previous constructions, covering all the QCD theories and mainly focusing on the $\frac{1}{2} \otimes \frac{1}{2}$ and $0 \otimes 1$ gravitational cases. This will allow us to gain further insight in the corresponding amplitudes. On the QCD side we will employ the compactification method to obtain the actions. On the gravity side we will construct them from simple considerations in the string frame, including classical regime. We will check our proposal using CHY-like formulas in appendix D. For two matter lines some of these Lagrangians acquire contact terms which we further study in section 4 .

\subsection{QCD theories}

We start by considering the QCD factors associated to the double copy. The cases of spin-0 and spin- $\frac{1}{2}$ are standard and we can provide the Lagrangian for more than one matter line straight away. The case of the QCD theory of spin-1 $[56,57]$ is more interesting and will be treated in a separate subsection.

\subsubsection{Spins $s=0, \frac{1}{2}$}

We have explained in the previous section how the scalar theory coupled to QCD arises from a particular compactification both in momenta and polarization vectors. The compactification in polarization vectors is obtained by considering a pure gluon amplitude and setting $\varepsilon_{i}=(0, \ldots 0 \mid 1)$ where the non-zero component explores an "internal space". We can immediately ask what happens if the internal space is enlarged to $N$ slots, namely the scalars are obtained by setting

$$
\varepsilon_{i}=(\underbrace{0, \ldots, 0}_{D} \mid \underbrace{0, \ldots 1, \ldots, 0}_{N}) .
$$


This construction is well known from string theory and the resulting amplitudes correspond to $N$ scalars in QCD. In other words, letting $I, J=1, \ldots, N$ the resulting amplitudes for any number of scalar lines are given by the aforementioned "special" Yang-Mills scalar theory:

$$
\mathcal{L}_{D}^{s=0}=-\frac{1}{4} \operatorname{tr}\left(F_{\mu \nu} F^{\mu \nu}\right)+\frac{1}{2} \operatorname{tr}\left(D_{\mu} \varphi_{I} D^{\mu} \varphi^{I}\right)-\frac{1}{4} \operatorname{tr}\left(\left[\varphi^{I}, \varphi^{J}\right]\left[\varphi_{I}, \varphi_{J}\right]\right) .
$$

The proof of this compactification is very simple and illustrative so we briefly outline it here. It follows from decomposing the gluon polarization in $D+N$ dimensions as

$$
A_{\mu} \rightarrow\left(A_{\mu} \mid \varphi_{1}, \ldots, \varphi_{N}\right)
$$

which implies

$$
F_{\mu I}=D_{\mu} \varphi_{I}, \quad F_{I J}=\left[\varphi_{I}, \varphi_{J}\right],
$$

together with the $D$ dimensional $F_{\mu \nu}$ components. Then, the resulting Lagrangian just follows from expanding $\operatorname{tr}\left(F^{2}\right)$. Note that the fields only depend on $D$ coordinates (see e.g. [110]). Two key remarks which will be useful later are as follows: first, the extra dimensional (scalar) modes are always pair-produced and hence will assemble into matter lines in the Feynman diagrams. In particular this means that even after dimensional reduction the pure gluon amplitudes coincide with the ones of YM theory. Second, as already pointed out in the original construction [108] of the compactified Yang-Mills action, the action (3.2) indeed corresponds to the bosonic sector of $\mathcal{N}=4$ Super Yang-Mills theory (in that case $D=4$ and $N=6$ ).

Let us now provide masses to the scalars in the Lagrangian (3.2). This requires to consider complex fields as is standard in KK reductions. There are a number of ways to achieve this. For instance, still following [108], we can consider an even number of compact dimensions $N$ after which the scalars can be grouped as $\psi=\varphi_{I}+i \varphi_{I+1}$.

Here we will instead take an alternative route that connects more directly to our previous amplitudes discussion, and therefore extends to particles with spin. Recall that so far we have constructed the double-copy formula for a single matter line (2.37). We can also consider scattering amplitudes for more matter lines as long as they have different flavors, a restriction that we impose throughout this paper. Now, for a given flavor $I$, the Lagrangian (3.2) takes the form $\mathcal{L}_{\mathcal{D}} \supset \frac{1}{2} \varphi_{I} \mathcal{D} \varphi^{I}$ (without summation) where $\mathcal{D}$ is a Hermitian operator that can depend on other fields. This Lagrangian generates the same Feynman rules than $\varphi_{I}^{*} \mathcal{D} \varphi^{I}$, which is the previous statement that the scalar fields are pairproduced. Repeating the argument for $I, J=1, \ldots, N$, we conclude that we can replace

$$
\mathcal{L}_{D}^{s=0} \rightarrow-\frac{1}{4} \operatorname{tr}\left(F_{\mu \nu} F^{\mu \nu}\right)+\operatorname{tr}\left(D_{\mu} \varphi_{I}^{*} D^{\mu} \varphi^{I}\right)-\operatorname{tr}\left(\left[\varphi^{* I}, \varphi^{* J}\right]\left[\varphi_{I}, \varphi_{J}\right]\right) .
$$

carrying a $\mathrm{U}(1)^{N}$ flavour. After providing masses to the complex fields, they can be turned into real fields again via the same argument. We will use this procedure in the remaining compactifications presented in this paper.

We now proceed then via KK reduction on a torus, $M_{D}=\mathbb{R}^{d} \times T^{N}$, and we let each of $N$ scalars to have a non-zero momentum in one of the circles $S^{1}$,

$$
\varphi_{I}(x, \theta)=e^{i m_{I} \theta_{I}} \varphi_{I}(x),
$$


where $0<\theta_{I} \leq \frac{2 \pi}{m_{I}}$. The gluon field has no momenta on $T^{N}$, i.e. is $\theta$-independent, and its only non-zero components are $A_{\mu}(x)$, where now $\mu=0, \ldots, d-1$. By acting with the derivative $\partial_{\bar{\mu}}$, where $\bar{\mu}=0, \ldots, D-1=d+N-1$, we can read off the momentum of the flavour $\varphi_{I}$ :

$$
p_{i \bar{\mu}}^{(I)}=(\underbrace{p_{i \mu}}_{d} \mid \underbrace{0, \ldots, m_{I}, \ldots, 0}_{N}) .
$$

Thus the on-shell condition becomes $\left(p_{i}^{I}\right)^{2}=p_{i}^{2}-m_{I}^{2}=0$ and, for $N=1$, this procedure is equivalent to the one described in the previous section. It generalizes it to more massive lines by imposing that the momenta of scalars of different flavour are orthogonal in the KK directions, i.e. $p_{i}^{(I)} \cdot p_{j}^{(J)}=p_{i} \cdot p_{j}$ for $I \neq J$. By integration on $T^{N}$ we find the corresponding massive action:

$$
\int d^{d} x d^{N} \theta \mathcal{L}_{D}^{s=0} \propto \int d^{d} x \operatorname{tr}\left(-\frac{1}{4} F_{\mu \nu} F^{\mu \nu}+\frac{1}{2} D_{\mu} \varphi_{I} D^{\mu} \varphi^{I}+\frac{1}{2} m_{I}^{2} \varphi_{I} \varphi^{I}-\frac{1}{4}\left[\varphi^{I}, \varphi^{J}\right]\left[\varphi_{I}, \varphi_{J}\right]\right),
$$

which corresponds to a scalar QCD theory, with a sum over flavours $I$ implicit. Here the scalars inherit the adjoint representation from the higher-dimensional gluons. For one matter line we can nevertheless take them in the fundamental representation (see section 3.1.2 below) and also drop the quartic term from the Lagrangian: the double copy of the resulting theory has been studied in [20] and we will come back to it in section 4 . On the other hand, by keeping the last term we have a non-trivial contact interaction between flavours. In the massless case the double copy of this theory with itself corresponds to Einstein-YM as first observed in [111]. In our case we will be interested in the double copy of (3.8) with the spin- 1 theory constructed in the next subsection, leading to the $0 \otimes 1$ gravitational theory. For two matter lines we shall see how the procedure applies both with and without the quartic interaction in (3.8), see sections 3.2.1 and 4.1.3 respectively. In the classical regime we also anticipate that this distinction is irrelevant and both cases can be regarded as equivalent.

Finally, we note that we can also apply the reduction procedure to massless QCD in order to get the massive theory, as discussed previously from the amplitudes perspective. Using the splitting (2.10) we obtain, after dropping some irrelevant KK modes,

$$
\int d^{d} x d^{N} \theta \mathcal{L}_{D}^{s=\frac{1}{2}} \propto \int d^{d} x \operatorname{tr}\left(-\frac{1}{4} F_{\mu \nu} F^{\mu \nu}+i \bar{\psi}_{I} \Gamma_{\mu} D^{\mu} \psi^{I}-m \bar{\psi}_{I} \psi^{I}\right) .
$$

In $d=4$ and for a single fermion line, we note that this reproduces the fermion amplitudes of $\mathcal{N}=4 \mathrm{SYM}$ in the Coulomb branch. This will be useful for performing the double copy via the CHY-like formalism introduced in [70, 71], which we do in appendix D.

\subsubsection{Spin $s=1$}

We now consider in detail the case of spin-1, that is, a complex Proca field coupled to QCD. In order to motivate this theory we will reproduce here the argument given by Holstein in [56] regarding the natural value of $g$, which we used in [47] to derive the three-point amplitude for spinning partilces in QED, but here we consider a slightly more general setup by promoting QED to QCD amplitudes. 
Consider first the (complex) Proca theory minimally coupled to $\mathrm{SU}(N)$ Yang-Mills theory,

$$
\mathcal{L}=-\frac{1}{4} F_{\mu \nu}^{a} F_{a}^{\mu \nu}-\frac{1}{4} W_{\mu \nu}^{\bar{I}} W_{I}^{\mu \nu}+\frac{m^{2}}{2} W_{\bar{I}}^{\mu} W_{\mu}^{I},
$$

where we have distinguished color indices $I, \bar{I}$ to emphasize that $\left(W^{\bar{I}}\right) W^{I}$ transforms in the (anti)fundamental representation. This is just a formal feature since for now we will only consider one matter line (note also that the mass does not depend on $I$ ). Here

$$
\begin{aligned}
W_{\mu \nu}^{I} & =D_{\mu} W_{\nu}^{I}-D_{\nu} W_{\mu}^{I}, \\
D_{\mu} W_{\nu}^{I} & =\partial_{\mu} W_{\nu}^{I}+A_{\mu}^{a} T_{a}^{I \bar{J}} W_{\nu \bar{J}} .
\end{aligned}
$$

Now consider the three point amplitude obtained from (3.10),

$$
A_{3}^{\mathrm{QCD}, 1}\left(W_{1}^{I} A_{3}^{a} W_{2}^{\bar{J}}\right)=2 T^{a I \bar{J}} \times\left(\epsilon_{3} \cdot p_{1} \varepsilon_{1} \cdot \varepsilon_{2}^{*}-\epsilon_{3 \mu} k_{3 \nu} \varepsilon_{1}^{[\mu} \varepsilon_{2}^{* \nu]}\right) .
$$

By recalling the example of (2.24) we can easily identify the scalar and dipole pieces in these two terms. Note that $\varepsilon_{1} \cdot J^{\mu \nu} \cdot \varepsilon_{2}^{*}=2 \varepsilon_{1}^{[\mu} \varepsilon_{2}^{* \nu]}$ and hence we obtain $g=1$. This is consistent with the value of $g=\frac{1}{s}$ obtained for minimally covariantized Lagrangians as conjectured by Belinfante [112]. We then proceed to modify the value of $g$ by adding the interaction

$$
\mathcal{L}_{\text {int }}=\beta F_{\mu \nu}^{a} T_{a}^{I \bar{J}} W_{I}^{\mu} W_{\bar{J}}^{\nu} .
$$

This interaction was studied in e.g. [56] restricted to the context of QED. In such case we can take $T_{a}^{I \bar{J}} \rightarrow \delta^{+-}$and $\mathcal{L}_{\text {int }}$ arises from the spontaneous symmetry breaking in the $W^{ \pm}$-boson model (with $\beta=1$ ). In our case we need to promote this to QCD so that we can perform the double copy at higher multiplicity. In any case, this term precisely deforms the value of the dipole interaction to $g=1+\beta$, because

$$
\mathcal{L}_{\text {int }} \rightarrow-2 \beta T_{a}^{I \bar{J}} \times \epsilon_{3 \mu} k_{3 \nu} \varepsilon_{1}^{[\mu} \varepsilon_{2}^{* \nu]} .
$$

Now, we claim that in order for $A_{3}^{\mathrm{QCD}}$ to be consistent with the double copy for the graviton states we will need to set $g=2$, i.e. $\beta=1$ as in the electroweak model. This is because only in such case we find ${ }^{13}$

$$
\begin{aligned}
A_{3}^{\mathrm{QCD}, 0} \times A_{3}^{\mathrm{QCD}, 1} & =A_{3}^{\mathrm{gr}, 1}\left(W_{1} h_{3} W_{2}\right) \\
& =\epsilon_{3} \cdot p_{1} \times\left(\epsilon_{3} \cdot p_{1} \varepsilon_{1} \cdot \varepsilon_{2}^{*}-2 \epsilon_{3 \mu} k_{3 \nu} \varepsilon_{1}^{[\mu} \varepsilon_{2}^{* \nu]}\right)
\end{aligned}
$$

Here we have stripped the coupling constants to make the comparison direct and written the graviton polarization as $\epsilon_{3}^{\mu \nu}=\epsilon_{3}^{\mu} \epsilon_{3}^{\nu}$ for simplicity, which can then be promoted to a general polarization $\epsilon_{3}^{\mu \nu}$. The fixing of $g=2$ follows then from the fact that gravitational amplitudes for any spin will always lead to $g=2$ as we outlined in the Compton example of section 2.2 .

\footnotetext{
${ }^{13}$ This is a slight simplification of the argument, which is what we used in [47] at $n=3$, arbitrary spin. Actually, Holstein [57] studied the double copy of $A_{4}^{\mathrm{QED}}$ with the purpose of showing the $1 / \mathrm{m}$ cancellations which are equivalent to $g=2$ as we saw in (2.44). Of course, the amplitude $A_{4}^{\text {gr }}$ did not feature any such divergences.
} 
The fact that the double copy is satisfied for the $W$-boson model but not for the "minimally coupled" Proca action is not a coincidence. As we have explained, the concept of minimal coupling that we attain here does not necessarily agree with the covariantization of derivatives in (3.10). Our condition for minimal coupling, and that of [53], is that the $m \rightarrow 0$ limit of $A_{n}^{\mathrm{QCD}}$ is well defined at any multiplicity $n$. The $W$-boson model arises from spontaneous symmetry breaking in $\mathrm{SU}(2)_{L} \times \mathrm{U}(1)_{Y}$ gauge theory, and as such, will be deformed back to Yang-Mills as we take $m \rightarrow 0$. This will precisely fix $\beta=1$ in (3.14) and we now show how.

From a Feynman diagram perspective, we have already explained how the QCD amplitudes we are after can be obtained from massive compactification of YM amplitudes. In the case of spin- 1 and a single matter line, we interpret the cubic Feynman diagrams of $A_{n}^{\mathrm{YM}}$ as associated to a color factor made of fundamental and adjoint structure constants, following [34]. As an example, for partial amplitudes in the half ladder (DDM) basis, we will consider the color factor associated to the ordering $\alpha=\left(1 \beta_{1} \ldots \beta_{n-2} 2\right)$ as

$$
f^{a_{1} a_{\beta_{1}} b_{1}} f^{b_{1} a_{\beta_{2}} b_{2}} \ldots f^{b_{n-3} a_{\beta_{n-2}} a_{2}} \rightarrow T_{a_{\beta_{1}}}^{I_{1} \bar{J}_{1}} T_{a_{\beta_{2}}}^{J_{1} \bar{J}_{2}} \ldots T_{a_{\beta_{n-2}}}^{J_{n-3} \bar{I}_{2}}
$$

where particles in $\left\{\beta_{1}, \ldots, \beta_{n}\right\}$ are gluons and particles 1 and 2 are bosons $W^{I_{1}}, W^{\bar{I}_{2}}$ respectively. The same operation can be repeated in any cubic color numerator of YM, which in general means to replace $f^{a b c} \rightarrow T_{a}^{I \bar{J}}$ for matter vertices or just leave them as $f^{a b c}$ for the 3 -gluon vertices. This means we identify three types of color indices: $A=(a, I, \bar{I}) .{ }^{14}$ After relabelling the structure constants and the fields accordingly, the field strength $\mathcal{F}_{\mu \nu}^{A}$ can be split into the components

$$
\mathcal{F}_{\mu \nu}^{a}=F_{\mu \nu}^{a}+2 T_{I \bar{J}}^{a} W_{[\mu}^{I} W_{\nu]}^{\bar{J}}, \quad \mathcal{F}_{\mu \nu}^{I}=W_{\mu \nu}^{I}, \quad \mathcal{F}_{\mu \nu}^{\bar{I}}=W_{\mu \nu}^{\bar{I}}
$$

where $W_{\mu \nu}$ is defined in (3.11). Now consider the YM action after relabelling

$$
\frac{1}{4} \mathcal{F}_{\mu \nu}^{A} \mathcal{F}_{A}^{\mu \nu}=\frac{1}{4} F_{\mu \nu}^{a} F_{a}^{\mu \nu}+\frac{1}{4} W_{\mu \nu}^{\bar{I}} W_{I}^{\mu \nu}+F_{a}^{\mu \nu} T_{I \bar{J}}^{a} W_{\mu}^{I} W_{\nu}^{\bar{J}}+\ldots
$$

where we have dropped the term with four $W$-bosons. Repeating the compactification procedure, this time on a single circle $S^{1}$, gives

$$
\mathcal{L}^{s=1}=-\frac{1}{4} F_{\mu \nu}^{a} F_{a}^{\mu \nu}-\frac{1}{4} W_{\mu \nu}^{\bar{I}} W_{I}^{\mu \nu}+\frac{m^{2}}{2} W_{\bar{I}}^{\mu} W_{\mu}^{I}-F_{a}^{\mu \nu} T_{I \bar{J}}^{a} W_{\mu}^{I} W_{\nu}^{\bar{J}},
$$

which is indeed the deformation of (3.10) by the "spin-dipole" coupling (3.13). Thus, we have shown that the massive spin-1 theory yielding the $g=2$ interaction when coupled to QCD is precisely the compactification of Yang-Mills theory for a single matter line, as described in section 2 .

\footnotetext{
${ }^{14}$ Formally we take $T_{a}^{I \bar{J}}=-T_{a}^{\bar{J} I}$ as in [34]. One must also be careful in that the structure constants $\left\{T_{a}^{I \bar{J}}, f^{a b c}\right\}$ do not form a Lie algebra (except in the $\mathrm{SU}(2)$ case) and hence cannot be used as an input to construct a pure YM action. However, the inconsistency appears in the Jacobi relation $T_{a}^{I \bar{J}} T_{a}^{K \bar{L}}+\ldots$ which is associated to two matter lines, which we are not interested here: we drop such interactions in our resulting Lagrangian.
} 


\subsection{Proposal for gravitational theories}

Let us now introduce the gravitational Lagrangians. We begin by a construction of both $0 \otimes 1$ and $\frac{1}{2} \otimes \frac{1}{2}$ theories in the string frame, following some simple guidelines. First, let us assume momentarily that the base massless theory, leading to the amplitudes $A_{n}^{\mathrm{gr}}\left(\gamma^{-} h_{3} \cdots h_{n} \gamma^{+}\right)$is indeed Einstein-Maxwell in both $\frac{1}{2} \otimes \frac{1}{2}$ and $0 \otimes 1$ cases,

$$
\mathcal{L}_{\text {base }}=-\sqrt{g}\left[\frac{2}{\kappa^{2}} R+\frac{1}{2} F_{\mu \nu}^{*} F^{\mu \nu}\right] .
$$

This allow us to signal the crucial difference between the $\frac{1}{2} \otimes \frac{1}{2}$ and $0 \otimes 1$ theories in the dilaton coupling. Following [113], in the string frame this can be generated by adding the kinetic term and promoting $\sqrt{g} \rightarrow \sqrt{g} e^{-\frac{\kappa}{2} \phi}$. Thus we propose

$$
\begin{aligned}
\mathcal{L}_{\text {base }}^{0 \otimes 1} & =\sqrt{g} e^{-\frac{\kappa}{2} \phi}\left[-\frac{2}{\kappa^{2}} R+\frac{1}{2}(\partial \phi)^{2}-\frac{1}{2} F_{\mu \nu}^{*} F^{\mu \nu}\right], \\
\mathcal{L}_{\text {base }}^{\frac{1}{2} \otimes \frac{1}{2}} & =\sqrt{g} e^{-\frac{\kappa}{2} \phi}\left[-\frac{2}{\kappa^{2}} R+\frac{1}{2}(\partial \phi)^{2}\right]-\sqrt{g} \times \frac{1}{2} F_{\mu \nu}^{*} F^{\mu \nu} .
\end{aligned}
$$

We now see the difference lies in the fact that the Maxwell term has been added before and after incorporating the dilaton, respectively. The coupling of the dilaton is simpler and in a sense trivial in the $\frac{1}{2} \otimes \frac{1}{2}$ theory, which is characteristic of the Brans-Dicke-Maxwell action [114]. In fact, we can take such theory into the so-called Jordan frame by setting

$$
\phi=-\frac{2}{\kappa} \ln \Phi,
$$

which leads to the standard Brans-Dicke theory [115]

$$
\mathcal{L}_{\text {base }}^{\frac{1}{2} \otimes \frac{1}{2}}=\frac{2}{\kappa^{2}} \sqrt{g}\left[-\Phi R+\frac{(\partial \Phi)^{2}}{\Phi}-\frac{\kappa^{2}}{2} \times \frac{1}{2} F_{\mu \nu}^{*} F^{\mu \nu}\right] .
$$

On the other hand, our proposal that the $0 \otimes 1$ action involves a non-trivial coupling to the dilaton arises from a careful consideration of the classical results of [26], which construction we further realize in section 4 as a double copy of a spinning source (e.g. $s=1$ ) in QCD with a scalar theory $(s=0)$.

At this point we can generate a mass term by performing the compactification on a circle, $M_{D}=\mathbb{R}^{d} \times S^{1}$, letting the Proca field to have a non-zero (quantized) momentum on $S^{1}$

$$
A_{\mu}(x, \theta)=e^{i m \theta} A_{\mu}(x),
$$

whereas the remaining fields have not, i.e. $h_{\mu \nu}(x)$ and $\phi(x)$ are $\theta$-independent. Notice we have also implicitly restricted the polarizations to lie in $d=D-1$ dimensions. For instance, the full metric reads

$$
g_{\bar{\mu} \bar{\nu}}=\eta_{\bar{\mu} \bar{\nu}}+\frac{\kappa}{2} h_{\bar{\mu} \bar{\nu}},
$$

but $h_{\bar{\mu} \bar{\nu}}$ only has non-zero components $h_{\mu \nu}$. This relies on the assumption, exemplified in section 3.1.1, that additional KK components will assemble into matter lines and hence can be decoupled. The only exception is the dilaton field, which would in principle obtain 
a contribution from the extra component $h_{D D}$ in $h_{\bar{\mu} \bar{\nu}}$. The reason we set this component to zero beforehand is precisely to reproduce our prescription (2.16) as opposed to (2.15) (which would lead to the standard dimensional reduction of the dilaton amplitudes).

After this clarification we can now readily perform the integration of the action over the compact direction, leading to

$$
\frac{1}{2 \pi} \int d^{d} x d \theta \mathcal{L}_{\text {base }}=\int d^{d} x \sqrt{g} \begin{cases}e^{-\frac{\kappa}{2} \phi}\left[-\frac{2}{\kappa^{2}} R-\frac{1}{2}(\partial \phi)^{2}-\frac{1}{2} F_{\mu \nu}^{*} F^{\mu \nu}+m^{2} A_{\mu}^{*} A^{\mu}\right] & , \text { for } 0 \otimes 1 \\ e^{-\frac{\kappa}{2} \phi}\left[-\frac{2}{\kappa^{2}} R-\frac{1}{2}(\partial \phi)^{2}\right]-\frac{1}{2} F_{\mu \nu}^{*} F^{\mu \nu}+m^{2} A_{\mu}^{*} A^{\mu} & , \text { for } \frac{1}{2} \otimes \frac{1}{2}\end{cases}
$$

The key point here is that we have performed the compactification in the string frame, where the dilaton coupling is trivial. We can move to the Einstein frame by setting $g_{\mu \nu} \rightarrow e^{-\frac{\kappa \phi}{d-2}} g_{\mu \nu}$. Perturbatively, this is equivalent to a change of basis in the asymptotic states, given by

$$
h_{\mu \nu} \rightarrow h_{\mu \nu}-\frac{\phi}{d-2} \eta_{\mu \nu}+\mathcal{O}(\kappa),
$$

which means the amplitudes in this frame can be computed as linear combinations of the string frame ones. Returning to the Lagrangian, we use

$$
R \rightarrow e^{-\frac{\kappa \phi}{d-2}}\left(R-\kappa \frac{d-1}{d-2} D^{2} \phi-\frac{d-1}{d-2} \frac{\kappa^{2}}{4} \partial_{\mu} \phi \partial^{\mu} \phi\right)
$$

after which we perform a trivial rescaling $(\phi \rightarrow(d-2) \phi)$ to get

$$
\mathcal{L}^{\frac{1}{2} \otimes \frac{1}{2}}=\sqrt{g}\left[-\frac{2}{\kappa^{2}} R+\frac{(d-2)}{2}(\partial \phi)^{2}-\frac{1}{2} e^{\frac{\kappa}{2}(d-4) \phi} F_{\mu \nu}^{*} F^{\mu \nu}+m^{2} e^{\frac{\kappa}{2}(d-2) \phi} A_{\mu}^{*} A^{\mu}\right],
$$

and

$$
\mathcal{L}^{0 \otimes 1}=\sqrt{g}\left[-\frac{2}{\kappa^{2}} R+\frac{(d-2)}{2}(\partial \phi)^{2}-\frac{1}{2} e^{-\kappa \phi} F_{\mu \nu}^{*} F^{\mu \nu}+m^{2} A_{\mu}^{*} A^{\mu}\right] .
$$

Note that only in $d=4$ the dilaton is not sourced by matter in the $\frac{1}{2} \otimes \frac{1}{2}$ theory. Indeed, consider momentarily the massless limit $m=0$. A general Einstein-Maxwell-Dilaton theory in four dimensions can be classified in the Einstein frame from the coupling $e^{-\kappa \alpha \phi} F^{2}$, with $0 \leq \alpha \leq \sqrt{3}[116,117]$. The Brans-Dicke theory corresponds to $\alpha=0$ whereas the low-energy limit of string theory yields $\alpha=1$. This is not surprising as we will soon identify the $0 \otimes 1$ with a dimensional extension of $\mathcal{N}=4$ Supergravity. We should mention that the $\alpha=\sqrt{3}$ case is characteristic of the well-known five dimensional KK theory, whose double copy structure was considered in [111].

These actions would be enough for amplitudes involving only gravitons, dilatons and two Proca fields as external states. However, in the case of the $0 \otimes 1$ theory we have seen that axions can be sourced by matter. Keeping the classical application in mind, this means that for two matter lines we will need to compute such contributions, as they will appear as virtual states. We begin by constructing the interaction that reproduces single matter-line amplitudes with external axions.

In order to introduce the axion coupling in the $0 \otimes 1$ theory we again resort to the classical results of [26], which found that in the string-frame the axion couples to the matter 
through

$$
\kappa \int d \tau H_{\mu \nu \rho} v^{\mu} S^{\nu \rho}
$$

Here $S^{\mu \nu}$ is the spin operator as introduced in section 2.2.1. This coupling can be reproduced in QFT by computing a "three-point" amplitude between the dipole and the axion,

$$
A_{3}^{\mu \nu} \propto \kappa p^{[\mu} \times S^{\nu] \rho} q_{\rho}
$$

where $q^{\mu}$ and $p^{\mu}$ are the momentum of the axion and the matter line respectively. As predicted, we identify the first factor as the scalar 3pt. amplitude $A_{3}^{\mu, s=0} \propto p^{\mu}$ and the second factor as the dipole of the spin-1 amplitude $\left.A_{3}^{\mu, s=1}\right|_{J} \propto S^{\mu \rho} q_{\rho}$ [47], which signals this corresponds to the $0 \otimes 1$ theory. The overall proportionality factor can be adjusted accordingly. The QFT 3-pt. vertex leading to (3.33) is then the direct analog of (3.32), That is, after identifying $S^{\mu \nu} \rightarrow J^{\mu \nu}$ up to longitudinal terms, (3.32) becomes

$$
-B_{\mu \nu}(q) \times \kappa p_{2}^{\mu} A^{*[\nu}\left(p_{2}\right) A^{\rho]}\left(p_{1}\right) q_{\rho} \rightarrow \frac{\kappa}{2} H_{\mu \nu \rho} \partial^{\mu} A^{*[\nu} A^{\rho]}=\frac{\kappa}{4} H_{\mu \nu \rho} A^{* \mu} F^{\nu \rho} .
$$

Attaching then the canonically normalized kinetic term $\frac{1}{6} H_{\mu \nu \rho} H^{\mu \nu \rho}$ we can readily take this vertex into the Einstein frame (also applying the aforementioned rescaling to $\phi$ ),

$$
\sqrt{g} e^{-\frac{\kappa}{2} \phi} \times \frac{1}{6} H_{\mu \nu \rho}\left(H^{\mu \nu \rho}+\frac{3 \kappa}{2}\left(A^{* \mu} F^{\nu \rho}+\text { c.c. }\right)\right) \rightarrow \sqrt{g} e^{-2 \kappa \phi} \times \frac{1}{6} H_{\mu \nu \rho}\left(H^{\mu \nu \rho}+\frac{3 \kappa}{2}\left(A^{* \mu} F^{\nu \rho}+\text { c.c. }\right)\right) .
$$

Note that this term is not deformed by the massive compactification since the derivatives in $F^{\mu \nu}$ are contracted with $H_{\mu \nu \rho}$ living in $d=D-1$ dimensions. We note that the complex character of the fields is important for the following compactification. However, once the compactification is done we are left with a quadratic action in the Proca field, which can then be turned into real invoking the argument above (3.5). Thus we finally arrive at the action principle presented in the introduction for one matter line:

$$
\mathcal{L}^{0 \otimes 1}=\sqrt{g}\left[-\frac{2 R}{\kappa^{2}}+\frac{(d-2)}{2}(\partial \phi)^{2}-\frac{e^{-2 \kappa \phi}}{6} H_{\mu \nu \rho}\left(H^{\mu \nu \rho}+\frac{3 \kappa}{2} A^{\mu} F^{\nu \rho}\right)-\frac{1}{4} e^{-\kappa \phi} F_{\mu \nu} F^{\mu \nu}+\frac{m^{2}}{2} A_{\mu} A^{\mu}\right]
$$

Note that the massless sector corresponds to $\mathcal{N}=0$ Supergravity [113] as seen also in [26]. We will rederive this result from a pure on-shell point of view in the following subsubsection, and extend it to two-matter lines. We will also perform various checks in our proposals for both $\frac{1}{2} \otimes \frac{1}{2}$ and $0 \otimes 1$ actions. We can also already draw some conclusion regarding the interactions: even though the axion is sourced by the Proca field, it is pair produced in the massless sector. This means that the axion is projected out in amplitudes involving external gravitons and dilatons with a single matter line, just as in the $\frac{1}{2} \otimes \frac{1}{2}$ theory. More importantly, an analogous reasoning can be applied to dilatons to show that in both $0 \otimes 1$ and $\frac{1}{2} \otimes \frac{1}{2}$ theories the graviton emission amplitudes are precisely the same, as we observed first in [47]. Now, as we have mentioned, when the dilaton is included as an external state its coupling differs in both theories: in particular, it follows from (3.30) that in the massless four dimensional case the dilaton is not sourced by the photon in the $\frac{1}{2} \otimes \frac{1}{2}$ theory, see e.g. the 4-pt. example in [34]. 


\subsubsection{Alternative construction of the $0 \otimes 1$ action}

From the identifications we have performed we can provide an additional argument to obtain $\mathcal{L}^{0 \otimes 1}$. First consider the massless case $m=0$. Then the $0 \otimes 1$ theory in any dimension is obtained from the massless version of (2.37); the double copy between scalar QCD $(s=0)$ and pure Yang-Mills $(s=1)$. In section 3.1.1 we have identified the scalar QCD theory as the bosonic sector of $\mathcal{N}=4 \mathrm{SYM}$ in four dimensions. It should be clear however that if these amplitudes are computed without imposing any kind of Gram identities (or plugging spinor-helicity variables) the resulting object is dimension independent and trivially extends the bosonic sector of $\mathcal{N}=4 \mathrm{SYM}$. This is can be achieved for instance by computing the compactification of YM explicitly via the CHY formulation [69] as in appendix D.

Now, as the double copy in (2.37) is precisely obtained via the standard massless KLT, we know that in four dimensions it yields [2]

$$
(\mathcal{N}=4 \mathrm{SUGRA})=(\mathcal{N}=4 \mathrm{SYM}) \otimes(\text { pure YM })
$$

Observe that the fermionic content in this theory only comes from the SYM factor. This means we can consistently truncate to the bosonic sector at both sides of the equality. Thus we learn that the $0 \otimes 1$ theory in four dimensions corresponds to the bosonic sector of $\mathcal{N}=4$ SUGRA specialized to a single matter line. Recall also that relation (3.37) follows from truncating the spectrum of $\mathcal{N}=8$ SUGRA, and the corresponding bosonic on-shell states are obtained as

$$
\left\{h^{\mu \nu}, B^{\mu \nu}, \phi\right\} \cup\left\{\gamma^{I \nu}\right\}=\left\{g^{\mu}, \phi^{I}\right\} \otimes\left\{\tilde{g}^{\nu}\right\},
$$

where $I=1, \ldots, 6$, with $\phi^{I}$ denoting six different adjoint scalars. Note that in this section we will consider a real photon or Proca field interacting gravitationally, this distinction is only relevant in QCD. Even though we have written the action for a single matter line, the fact the spectrum has flavoured fields suggests that we can promote the construction to more matter lines and yet employ the standard KLT kernel as we will explain shortly. In the next section we will contrast this with the $\frac{1}{2} \otimes \frac{1}{2}$ theory and a different approach to the $0 \otimes 1$ construction obtained via the BCJ prescription. However, this example will already illustrate an important feature of these constructions: in general we will find matter contact interactions when more massive lines are included; these contact interactions however will not affect the classical limit.

Our strategy is analogous to the one in section 2. We first rewrite $\mathcal{N}=4$ SUGRA in a way in which the action is not sensitive to the dimension $d$. We then uplift it to general $d$ and compactify it on a torus. Starting from the standard Lagrangian given in [74, 75], i.e.

$$
\mathcal{L}^{\mathcal{N}=4}=\sqrt{g}\left[R-2(\partial \phi)^{2}-2 e^{4 \phi}(\partial \chi)^{2}-e^{-2 \phi} F_{\mu \nu}^{I} F_{I}^{\mu \nu}-2 \chi F_{\mu \nu}^{I} \star F_{I}^{\mu \nu}\right]
$$

where $\chi$ is the dual axion field, we review in appendix $\mathrm{C}$ the construction of Nicolai and Townsend and obtain

$$
\sqrt{g}\left[R-2(\partial \phi)^{2}+3 e^{-4 \phi}\left(A_{I}^{\nu} F^{I \rho \sigma}+\frac{1}{6} H^{\nu \rho \sigma}\right)\left(A_{J \nu} F_{\rho \sigma}^{J}+\frac{1}{6} H_{\nu \rho \sigma}\right)-e^{-2 \phi} F_{\mu \nu}^{I} F_{I}^{\mu \nu}\right],
$$


from dualizing the axion off-shell. This generates the term $\sim A^{2} F^{2}$, representing a new contact interaction between flavours which will appear for two matter lines. Even though this action makes sense in any dimension, we know it is in principle still four dimensional. To obtain a faithful dimensional continuation of $\mathcal{N}=4$ SUGRA we need to show that the amplitudes are indeed dimension independent. By looking at $2 \rightarrow 2$ scattering of photons (i.e. Proca fields) we can conjecture that this is achieved by promoting the kinetic term of the dilaton as $2(\partial \phi)^{2} \rightarrow(d-2)(\partial \phi)^{2}$ (such that it cancels the factor $\frac{1}{d-2}$ in the graviton propagator). This is exactly what we obtained from adopting the Einstein frame in the previous subsection. We can now swap to the string frame and perform the compactification on a torus $T^{6}$ as in the scalar case. After checking that the term $A_{I}^{\nu *} F^{I \rho \sigma} A_{J \nu}^{*} F_{\rho \sigma}^{J}$ will not generate mass dependence we get to the following form

$$
\sqrt{g}\left[R-(d-2)(\partial \phi)^{2}+3 e^{-4 \phi}\left(A_{I}^{\nu} F^{I \rho \sigma}+\frac{1}{6} H^{\nu \rho \sigma}\right)\left(A_{J \nu} F_{\rho \sigma}^{J}+\frac{H_{\nu \rho \sigma}}{6}\right)-e^{-2 \phi} F_{\mu \nu}^{I} F_{I}^{\mu \nu}+2 m_{I}^{2} A_{\mu}^{I} A_{I}^{\mu}\right]
$$

(recall that we will only consider amplitudes for Proca pairs of different flavours). In order to construct perturbation theory in the gravitational constant $\kappa$ we scale $\phi \rightarrow \frac{\kappa}{2} \phi, B_{\mu \nu} \rightarrow$ $\kappa B_{\mu \nu}, A_{\mu} \rightarrow \frac{\kappa}{2 \sqrt{2}} A_{\mu}$ and attach and overall factor of $-\frac{2}{\kappa^{2}}$ leading to

$$
\begin{aligned}
\sqrt{g}[ & -\frac{2}{\kappa^{2}} R+\frac{(d-2)}{2}(\partial \phi)^{2}-\frac{1}{6} e^{-2 \kappa \phi}\left(\frac{3 \kappa}{4} A_{I}^{\nu} F^{I \rho \sigma}+H^{\nu \rho \sigma}\right)\left(\frac{3 \kappa}{4} A_{J \nu} F_{\rho \sigma}^{J}+H_{\nu \rho \sigma}\right) \\
& \left.-\frac{1}{4} e^{-\kappa \phi} F_{\mu \nu}^{I} F_{I}^{\mu \nu}+\frac{1}{2} m_{I}^{2} A_{\mu}^{I} A_{I}^{\mu}\right] .
\end{aligned}
$$

For one matter line this precisely agrees with (3.36). For more Proca fields it features the aforementioned contact interaction. Note that amplitudes for (3.42) can be computed by again compactifying the KLT relation, where the massive photons in (3.38) appear as products of massive scalars with massive $W$-bosons, see appendix D.1 for an example with two matter lines. ${ }^{15}$ Both the massive scalar and the $W$-boson theory will as well contain interactions between flavours: in the massive scalar case, recall the special YMS action (3.8) which included a quartic term for scalars. In the W-boson case, recall that in section 3.1.2 we established the compactification of YM into the spin-1 theory only for a single matter line. This is precisely because for two matter lines the following diagram appears from compactification (the KK momenta are given by the masses $m_{a}$ and $m_{b}$ ):

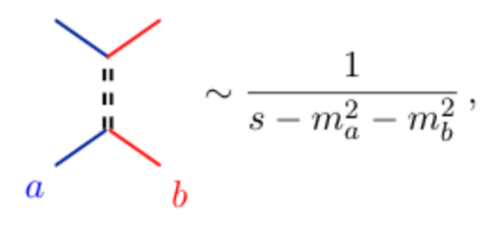

where two interacting flavours generate an extra massive particle with $M^{2}=m_{a}^{2}+m_{b}^{2}$. This pole is however cancelled by the KLT kernel yielding only contact interactions between flavours in (3.42).

\footnotetext{
${ }^{15}$ This example will also illustrate how the prescription (2.16) for dilaton dimensional reduction is automatically implemented also for internal dilatons. Namely, the KK components that could have contributed to internal dilatons decouple because they are orthogonal for different flavor lines.
} 
At this point it may be desirable to remove flavor interactions such as the ones present in diagram (3.43), hence also removing the extra massive pole. In the massless theory this diagram is required by gauge invariance, but given that for massive particles this is not a constraint the truncation would in principle define valid tree-level amplitudes. However, one must be careful in that without gauge invariance the longitudinal modes $\epsilon^{\mu}=\frac{p^{\mu}}{m}$ may lead to divergent amplitudes as we approach back to the massless case $m \rightarrow 0 .{ }^{16}$ On the other hand, it is known that flavor interactions such as (3.43) will not contribute to the classical regime (e.g. to a long-range potential) and hence the truncated amplitudes should lead to the same classical observables as the original theory, which will indeed be finite as $m \rightarrow 0$. Motivated by this, in the next section we will construct an alternative double copy for $0 \otimes 1$ at two matter lines, leading to the same classical observables as the extension of $\mathcal{N}=4$ SUGRA.

Despite the evidence we have presented so far it is important to perform explicit checks on our proposed Lagrangians for both $0 \otimes 1$ and $\frac{1}{2} \otimes \frac{1}{2}$ theories. We have checked explicitly the amplitudes, in arbitrary dimension, with all possible combinations of external states for $n=3,4,5$ points, including the two matter-line cases presented in section 4 . We have also performed six point checks for dilaton amplitudes, allowing us to test the exponential in (3.30) up to fourth order. The analytical results for some of these checks are too long to be printed in this paper, we therefore provide a Mathematica notebook with those amplitudes. These checks were also done efficiently via the CHY-like implementations we detail in appendix D.

\section{Two matter lines from the BCJ construction}

So far we have used the KLT double copy mostly to compute the amplitudes $A_{n}$, i.e. those involving one matter line. To test the extent of the double copy it is important to include more matter lines transforming in the fundamental representation. In our case it will be enough to consider two matter lines of different flavours in order to make contact with the classical results mentioned in the introduction. These amplitudes lose many nice features of the $A_{n}$ amplitudes: for instance we cannot trivially remove the dilaton-axion propagation nor write the multipole expansion directly. We shall anyhow conclude that the relevant classical information is already contained in the $A_{n}$ amplitudes, as pointed out in e.g. [48], which we have used to remove the dilaton/axion from the classical perspective in [47].

For more than one matter line a basis of amplitudes based on Dyck words was introduced by Melia $[118,119]$ and later refined by Johansson and Ochirov [35, 120]. ${ }^{17}$ Since we only consider here two matter lines we choose to resort instead to the BCJ representation, thereby extending the approach of [20]. The equivalence between the approaches has been detailed, including spin- $\frac{1}{2}$ applications, in e.g. [37].

\footnotetext{
${ }^{16}$ We thank A. Ochirov and H. Johansson for this observation, see also the introduction of [79].

${ }^{17}$ The amplitudes in Melia basis satisfy a restricted set of BCJ relations [35, 36], and consequently a generalized KLT construction has been recently introduced in [38, 79], see also [37]. For loop level extensions of colour-kinematics duality in this context see [121-124].
} 
Consider the two matter lines to have mass $m_{a}$ and $m_{b}$, and spin $s_{a}$ and $s_{b}$. For QCD scattering, the two massive particles have different flavours, and we restrict their spins to lie in $\left\{0, \frac{1}{2}, 1\right\}$. These amplitudes are defined by the Lagrangians provided in section 3: for the spin-0 case we use the scalar QCD Lagrangian (3.8) with the removed quartic term as per our previous discussion; for spin- 1 we use the $W$-boson model (3.19) and for spin- $1 / 2$ we use the standard QCD Lagrangian for massive Dirac fermions (3.9).

Following the BCJ prescription we arrange the QCD amplitudes into a sum of the form

$$
M_{n}^{\mathrm{QCD}}=\sum_{i \in \Gamma} \frac{c_{i} n_{i}^{\left(s_{a}, s_{b}\right)}}{d_{i}},
$$

running over the set $\Gamma$ of all cubic diagrams, with denominators $d_{i}$. The superscript $\left(s_{a}, s_{b}\right)$ here denotes the spin of the lines and may be omitted. For a given triplet $(i, j, k)$, if the color factors satisfy the Jacobi identity

$$
c_{i} \pm c_{j}= \pm c_{k},
$$

then colour kinematics duality requires there is a choice of numerators $n_{i}$ such that

$$
n_{i} \pm n_{j}= \pm n_{k}
$$

The gravitational amplitudes can be computed starting from (4.1) by replacing the color factors with further kinematic factors, which can be associated to a different QCD theory. In this section we will explore some of the choices for QCD theories, and write the explicit form of the resulting gravitational Lagrangians. With this in mind, the $n$-point gravitational amplitude, where now the massive lines have spins $s_{a}+\tilde{s}_{a}$ and $s_{b}+\tilde{s}_{b}$ respectively, reads

$$
M_{n}^{\left(s_{a} \otimes \tilde{s}_{a}, s_{b} \otimes \tilde{s}_{b}\right)}=\sum_{i \in \Gamma} \frac{n_{i}^{\left(s_{a}, s_{b}\right)} \otimes \tilde{n}_{i}^{\left(\tilde{s}_{a}, \tilde{s}_{b}\right)}}{d_{i}},
$$

where the product $\otimes$ depends on the spin of the massive particles in the QCD theory. For instance, for $s_{a}=\tilde{s}_{a}=s_{b}=\tilde{s}_{b}=1 / 2$ we define it in an analogous way to the case of only one matter line (2.13); that is: consider the spin $\frac{1}{2}$ operators $\mathcal{X}_{i}$ and $\mathcal{Y}_{i}$, entering in a QCD numerator $n^{\mathrm{QCD}}$ with four external fermions whose momenta we choose to be all outgoing as follows

$$
n^{\left(\frac{1}{2}, \frac{1}{2}\right)}=\bar{u}_{2} \mathcal{X}_{i} v_{1} \bar{u}_{4} \mathcal{Y}_{i} v_{3}
$$

analogously, the charge conjugated numerator reads

$$
\bar{n}^{\left(\frac{1}{2}, \frac{1}{2}\right)}=\bar{u}_{1} \overline{\mathcal{X}}_{i} v_{2} \bar{u}_{3} \overline{\mathcal{Y}}_{i} v_{4}
$$

We define the spin-1 gravitational numerator as the tensor product of the two QCD numerators as follows:

$$
n^{\left(\frac{1}{2}, \frac{1}{2}\right)} \otimes \bar{n}^{\left(\frac{1}{2}, \frac{1}{2}\right)}=\frac{1}{2^{2\lfloor d / 2\rfloor-2}} \operatorname{tr}\left[\mathcal{X}_{i \phi_{1}}\left(\not p_{1}+m_{a}\right) \overline{\mathcal{X}}_{i} \ddagger_{i}\left(\not p_{2}+m_{a}\right)\right] \operatorname{tr}\left[\mathcal{Y}_{i} \notin_{3}\left(\not p_{3}+m_{b}\right) \overline{\mathcal{Y}}_{i} \ddagger_{4}\left(\not p_{4}+m_{b}\right)\right],
$$


Notice that the generalization of (4.7) to an arbitrary number of massive lines could be done analogously by introducing one Dirac trace for each matter line.

In this section we focus on elastic scattering, given by $M_{4}$, and inelastic scattering, given by $M_{5}$, firstly from a QFT perspective and then from a classical perspective. Nevertheless, we propose Lagrangians for arbitrary multiplicity as long as we keep two matter lines.

Setting conventions, the momenta of the particles are taken as follows: for the $2 \rightarrow 2$ elastic scattering, the two incoming momenta are $p_{1}$ and $p_{3}$, and the outgoing momenta are $p_{2}=p_{1}-q$ and $p_{4}=p_{3}+q$, for $q$ the momentum transfer. For the $2 \rightarrow 3$ inelastic scattering, again the two incoming momenta are $p_{1}$ and $p_{3}$, whereas the momenta for the two outgoing massive particles are $p_{2}=p_{1}-q_{1}$ and $p_{4}=p_{3}-q_{3}$, and the outgoing gluon or graviton has momentum $k$.

\subsection{Elastic scattering}

The simplest example of the scattering of two massive particles of mass $m_{a}$ and $m_{b}$, and spin $s_{a}$ and $s_{b}$, is the elastic scattering, which we call $M_{4}^{\left(s_{a}, s_{b}\right)}$ amplitudes. Let us illustrate how the double copy works for some choices of $s_{a}$ and $s_{b}$.

\subsubsection{Spinless case}

As a warm up consider the case of two scalar particles of incoming momenta $p_{1}$ and $p_{3}$, and momentum transfer $q$. The gravitational amplitude (4.4) can be computed from the double copy of the gluon exchange amplitude between two scalar particles. The kinematic numerator and denominator for the gauge theory have the explicit form

$$
n^{(0,0)}=-e^{2}\left(4 p_{1} \cdot p_{3}+q^{2}\right), \quad d_{4}=q^{2} .
$$

In this case there is no kinematic Jacobi identities (4.3), hence color-kinematics duality is trivially satisfied. It is therefore straightforward to use the double copy (4.4) to write the gravitational amplitude

$$
M_{4}^{(0 \otimes 0,0 \otimes 0)}=\frac{\kappa^{2}}{16} \frac{\left(4 p_{1} \cdot p_{3}+q^{2}\right)^{2}}{q^{2}} .
$$

As the double copy is symmetric in the two numerators, by looking at the cut $q^{2} \rightarrow 0$ we can see that the axion field does not propagate and instead there is only the propagation of the graviton and the dilaton. Also, the fact that the amplitude is a perfect square is non-trivial from a Feynman diagram perspective. This factorization can be understood by decomposing (4.9) into three pieces:

$$
\frac{\left(4 p_{1} \cdot p_{3}+q^{2}\right)^{2}}{q^{2}}=\underbrace{\frac{\left(4 p_{1} \cdot p_{3}+q^{2}\right)^{2}-8 m_{a}^{2} m_{b}^{2}-q^{4}}{q^{2}}}_{\text {Pure Gravity }}+\underbrace{\frac{8 m_{a}^{2} m_{b}^{2}}{q^{2}}}_{\phi \text { exchange }}+\underbrace{q^{2}}_{\text {contact term }}
$$

Thus, we find that in order to achieve the factorization a quartic term must be included in the action. This is a general feature even with spin. 
Likewise the one matter line case, we can write a gravitational Lagrangian from which the amplitude (4.9) can be computed. In the Einstein frame it takes the form

$$
\mathcal{L}^{(0 \otimes 0,0 \otimes 0)}=\sqrt{g}\left[-\frac{2}{\kappa^{2}} R+\frac{2(d-2)}{\kappa^{2}}(\partial \phi)^{2}+\frac{1}{2}\left(\partial \varphi_{I}\right)^{2}+\frac{1}{2} e^{-2 \phi} m_{I}^{2} \varphi_{I}^{2}+\frac{\kappa^{2}}{16} \varphi_{1} \varphi_{2}\left(\partial \varphi_{1}\right) \cdot\left(\partial \varphi_{2}\right)\right],
$$

which we call the scalar-gravitational theory. Here the $\varphi_{I}$ fields correspond to the two massive scalars fields, and it is understood that $I \in\{1,2\}$, with $m_{1}=m_{a}$ and $m_{2}=m_{b}$.

Guided from the decomposition (4.10), we have introduce a contact interaction term in the Lagrangian to match precisely the double copy result. We have implicitly extended this contact term to arbitrary multiplicity by providing a covariant action. We will find that this covariantization is respected when computing the double copy for the inelastic amplitude, $M_{5}^{(0 \otimes 0,0 \otimes 0)}$.

As a final short remark, let us point out that the contact interaction does not contribute to the classical limit (see section 4.3 for its definition). Indeed, by removing the dilaton exchange, the first and last terms of (4.10) were used in [20] to recover classical gravitational radiation.

\subsubsection{Case $s_{a}=0+1$ and $s_{b}=0+0$}

Now we can add spin to one of the massive lines. In the gravitational theory, particle $a$ has spin 1 , whereas particle $b$ remains spinless. The gravitational amplitude (4.4) for this case can be computed from the double copy of the scalar numerators (4.8) and the numerator $n^{(1,0)}$. The later corresponds to the gluon exchange between a scalar and a spin-1 particle, using the interactions from (3.8) and (3.19). It takes the simple form

$$
n^{(1,0)}=-e^{2}\left[\left(4 p_{1} \cdot p_{3}+q^{2}\right) \varepsilon_{1} \cdot \varepsilon_{2}-4\left(p_{3} \cdot \varepsilon_{2} q \cdot \varepsilon_{1}-p_{1} \cdot \varepsilon_{2} p_{3} \cdot \varepsilon_{1}\right)\right],
$$

where $\varepsilon_{1(2)}$ is the polarization vector for the incoming (outgoing) spining particle. This is precisely the full numerator used in e.g. [125]. However, we warn the reader that it arises from a single matter line truncation of Yang-Mills theory (3.19) and hence should be regarded only as a thoroughfare to the classical limit, as discussed below eq. (3.43).

The simplicity of the construction enables us to readily write down a Lagrangian for the double copy. More precisely, an analogous decomposition to (4.10) of the gravitational amplitude allows us to identify the contact interaction. The gravitational Lagrangian in the Einstein frame is simply given by

$$
\begin{aligned}
\mathcal{L}^{(0 \otimes 1,0 \otimes 0)}=\sqrt{g}[ & -\frac{2}{\kappa^{2}} R+\frac{2(d-2)}{\kappa^{2}}(\partial \phi)^{2}-\frac{1}{4} e^{-2 \phi} F_{\mu \nu} F^{\mu \nu}+\frac{m_{a}^{2}}{2} A_{\mu} A^{\mu}+\frac{1}{2}(\partial \varphi)^{2} \\
& \left.+\frac{1}{2} m_{b}^{2} e^{-2 \phi} \varphi^{2}+\frac{\kappa^{2}}{16}\left(2 A^{\mu} \partial_{\mu} \varphi A^{\nu} \partial_{\nu} \varphi+\varphi A^{\nu} \partial_{\mu} \varphi \partial^{\mu} A_{\nu}\right)\right]
\end{aligned}
$$

where $A_{\mu}$ corresponds to the massive spin- 1 field and $\varphi$ is the massive scalar field. Again, we will find that the covariantization of the contact term will be confirmed by the respective $M_{5}$ and we conjecture the same for higher multiplicity. Indeed, we have already found the term $A^{\mu} \partial_{\mu} \varphi A^{\nu} \partial_{\nu} \varphi$ previously! We obtained it in [47] and identified it as certain quantum contributions to the quadrupole arising in our classical double copy. 


\subsubsection{Case $s_{a}=s_{b}=0+1$}

The natural generalization of the previous cases is to consider that both matter lines have spin-1 in the gravitational theory. For this case there are two possible theories: the first one is dictated by the factorization $s_{a}=s_{b}=0+1$, whereas for the second theory, the factorization is $s_{a}=s_{b}=\frac{1}{2}+\frac{1}{2}$. In this subsection we consider the former, and leave the latter to be explored in the next subsection.

The construction we consider here is an alternative to the two matter lines amplitudes obtained from (3.42), i.e. the extension of $\mathcal{N}=4$ SUGRA. The difference lies in that here we will chop the flavour-interaction terms in the QCD Lagrangians, also leading to no such interaction on the gravity side. As explained below (3.43) this prevents the appearance of the additional massive particles arising in the dimensional reduction of $\mathcal{N}=4$ SUGRA. It is also interesting to explore because for only one line with spin we have found that this truncation leads to a simplified double copy construction.

The gravitational scattering amplitude (4.4) at four points can be obtained from the double copy of the scalar numerators (4.8) and the spin-1 numerator $n^{(1,1)}$. This numerator can be computed from the gluon exchange between two massive spin-1 fields, each described by the matter part of (3.19), and results into

$$
\begin{aligned}
n^{(1,1)}=-4 e^{2}[ & \frac{1}{4}\left(4 p_{1} \cdot p_{3}+q^{2}\right) \varepsilon_{1} \cdot \varepsilon_{2} \varepsilon_{3} \cdot \varepsilon_{4}-\left(p_{1} \cdot \varepsilon_{3} p_{3} \cdot \varepsilon_{4}+p_{1} \cdot \varepsilon_{3} q \cdot \varepsilon_{4}\right) \varepsilon_{1} \cdot \varepsilon_{2} \\
& -\left(p_{1} \cdot \varepsilon_{2} p_{3} \cdot \varepsilon_{1}-p_{3} \cdot \varepsilon_{2} q \cdot \varepsilon_{1}\right) \varepsilon_{3} \cdot \varepsilon_{4}+p_{1} \cdot \varepsilon_{2} p_{3} \cdot \varepsilon_{4} \varepsilon_{1} \cdot \varepsilon_{3} \\
& \left.-q \cdot \varepsilon_{1} q \cdot \varepsilon_{3} \varepsilon_{2} \cdot \varepsilon_{4}-p_{3} \cdot \varepsilon_{4} q \cdot \varepsilon_{1} \varepsilon_{2} \cdot \varepsilon_{3}+p_{1} \cdot \varepsilon_{2} q \cdot \varepsilon_{3} \varepsilon_{1} \cdot \varepsilon_{4}\right] .
\end{aligned}
$$

The gravitational Lagrangian for this theory has a more intricate structure than the ones we have considered so far for the case of two matter lines, which is natural due to additional propagation of the axion coupling to the spin of the matter lines. It can be shown that the Lagrangian is given by

$$
\begin{aligned}
\mathcal{L}^{(0 \otimes 1,0 \otimes 1)}=\mathcal{L}_{c t}+\sqrt{g} & {\left[-\frac{2}{\kappa^{2}} R+\frac{2(d-2)}{\kappa^{2}}(\partial \phi)^{2}-\frac{e^{-4 \phi}}{6 \kappa^{2}} H_{\mu \nu \rho} H^{\mu \nu \rho}\right.} \\
& \left.-\frac{e^{-4 \phi}}{6 \kappa^{2}} H_{\mu \nu \rho} A_{I}^{\mu} F^{I \nu \rho}-\frac{1}{4} e^{-2 \phi} F_{I, \mu \nu} F^{I \mu \nu}+\frac{m_{I}^{2}}{2} A_{I, \mu} A^{I \mu}\right],
\end{aligned}
$$

where the flavour index $I \in\{1,2\}$, and once again the masses $m_{1}=m_{a}$ and $m_{2}=m_{b}$. The contact interaction Lagrangian for this case has the form

$$
\begin{aligned}
\mathcal{L}_{c t} \sim \sqrt{g}\left[2 A_{1} \cdot A_{2}\left(\partial_{\mu} A_{1, \nu}-3 \partial_{\nu} A_{1, \mu}\right) \partial^{\mu} A_{2}^{\nu}-2 A_{2} \cdot F_{1} \cdot F_{2} \cdot A_{1}\right. \\
\\
\left.\quad-2 A_{2}^{\mu} \partial_{\mu} A_{1}^{\alpha} A_{2}^{\nu} \partial_{\nu} A_{1, \alpha}-A_{1}^{\mu} \partial_{\mu} A_{2}^{\alpha} A_{1}^{\nu} \partial_{\nu} A_{2 \alpha}-A_{1}^{\mu} \partial_{\alpha} A_{1, \mu} A_{2}^{\nu} \partial^{\alpha} A_{2, \nu}\right]
\end{aligned}
$$

where the product of field strength tensors reads explicitly

$$
\begin{aligned}
A_{2} \cdot F_{1} \cdot F_{2} \cdot A_{1}= & A_{2}^{\mu} \partial_{\mu} A_{1}^{\alpha} \partial_{\alpha} A_{2, \nu} A_{1}^{\nu}-A_{2}^{\mu} \partial^{\alpha} A_{1, \mu} \partial_{\alpha} A_{2, \nu} A_{1}^{\nu} \\
& -A_{2}^{\mu} \partial_{\mu} A_{1}^{\alpha} \partial_{\nu} A_{2, \alpha} A_{1}^{\nu}-A_{2}^{\mu} \partial^{\alpha} A_{1, \mu} \partial_{\nu} A_{2, \alpha} A_{1}^{\nu} .
\end{aligned}
$$

Thus in this case, for two particles including spin, we have found an elevated level of complexity even for the four-point terms in the Lagrangian. Because of this in the following 
we simply resort to the extension of $\mathcal{N}=4$ SUGRA, eq. (3.42), in order to write the interaction Lagrangian for two or more matter lines. Once again, the same classical limit of the amplitude can be obtained from the above construction (i.e. via (4.4)) or from the extension of $\mathcal{N}=4$ SUGRA (i.e. via KLT), the equivalence of which will prove useful for inelastic scattering in the next section.

\subsubsection{Case $s_{a}=s_{b}=\frac{1}{2}+\frac{1}{2}$}

We finish the discussion for the elastic scattering considering the simplest gravitational theory for both of the massive lines with spin-1. As we mentioned previously, this theory is dictated by the factorization $s_{a}=s_{b}=\frac{1}{2}+\frac{1}{2}$. The gravity amplitude (4.4) at 4 pt. is computed from the double copy of the QCD spin $\frac{1}{2}$ numerator $n^{\left(\frac{1}{2}, \frac{1}{2}\right)}$, and its charge conjugated pair. They have a simple form

$$
\begin{aligned}
& n^{\left(\frac{1}{2}, \frac{1}{2}\right)}=e^{2} \bar{u}_{2} \gamma^{\mu} u_{1} \bar{u}_{4} \gamma_{\mu} u_{3}, \\
& \bar{n}^{\left(\frac{1}{2}, \frac{1}{2}\right)}=e^{2} \bar{v}_{1} \gamma^{\mu} v_{2} \bar{v}_{3} \gamma_{\mu} v_{4},
\end{aligned}
$$

where we use the condition for momentum conservation $p_{2}=p_{1}-q$ and $p_{4}=p_{3}+q$. Now, using the double copy operation for two matter lines (4.7), the gravitational amplitude takes the compact form

$$
M_{4}^{\left(\frac{1}{2} \otimes \frac{1}{2}, \frac{1}{2} \otimes \frac{1}{2}\right)}=\frac{4}{2^{2\lfloor D / 2\rfloor}} \frac{\kappa^{2}}{q^{2}} \operatorname{tr}\left[\gamma^{\mu} \ddagger_{1}\left(\not p_{1}-m_{a}\right) \gamma^{\nu} \ddagger_{2}\left(\not p_{2}+m_{a}\right)\right] \operatorname{tr}\left[\gamma_{\mu} \ddagger_{3}\left(\not p_{3}-m_{b}\right) \gamma_{\nu} \ddagger_{4}\left(\not p_{4}+m_{b}\right)\right],
$$

Notice the momenta $p_{1}$ and $p_{3}$ are incoming, therefore the sign in the projector changes. After taking the traces the amplitude reads

$$
\begin{aligned}
M_{4}^{\left(\frac{1}{2} \otimes \frac{1}{2}, \frac{1}{2} \otimes \frac{1}{2}\right)}= & \frac{4 \kappa^{2}}{q^{2}}\left\{\left[\varepsilon_{1} \cdot \varepsilon_{2}\left((d-6) p_{1}^{\nu} p_{2}^{\mu}+(d-2) p_{1}^{\mu} p_{2}^{\nu}\right)-p_{1} \cdot \varepsilon_{2}\left((d-6) \varepsilon_{1}^{\nu} p_{2}^{\mu}+(d-2) \varepsilon_{1}^{\mu} p_{2}^{\nu}\right)\right.\right. \\
& -p_{2} \cdot \varepsilon_{1}\left((d-6) p_{1}^{\nu} \varepsilon_{2}^{\mu}+(d-2) p_{1}^{\mu} \varepsilon_{2}^{\nu}\right)+\left((d-6) p_{1} \cdot p_{2}+(d-4) m_{a}^{2}\right)\left(\varepsilon_{1}^{\mu} \varepsilon_{2}^{\nu}-\varepsilon_{1} \cdot \varepsilon_{2} \eta^{\mu \nu}\right) \\
& \left.\left.+\left((d-2) p_{1} \cdot p_{2}+d m_{a}^{2}\right) \varepsilon_{1}^{\mu} \varepsilon_{2}^{\nu}+(d-6) p_{1} \cdot \varepsilon_{2} p_{2} \cdot \varepsilon_{1} \eta^{\mu \nu}\right] \times[\text { line } a \rightarrow \text { line } b]_{\mu \nu}\right\},
\end{aligned}
$$

where the change [line $a \rightarrow$ line $b]$ means to do $[1 \rightarrow 3,2 \rightarrow 4, a \rightarrow b]$. Likewise for the two previous cases, we can write the gravitational Lagrangian for this theory, surprisingly it has a very simple form

$$
\mathcal{L}^{\left(\frac{1}{2} \otimes \frac{1}{2}, \frac{1}{2} \otimes \frac{1}{2}\right)}=\sqrt{g}\left[-\frac{2}{\kappa^{2}} R+\frac{2(d-2)}{\kappa^{2}}(\partial \phi)^{2}-\frac{1}{4} e^{(d-4) \phi} F_{I, \mu \nu} F_{I,}^{\mu \nu}+\frac{1}{2} e^{(d-2) \phi} m_{I}^{2} A_{I \mu} A_{I}^{\mu}\right],
$$

We say that this is the simplest theory for spinning particles coupled to gravity in two senses: first, even thought the two massive lines have spin, there is no propagation of the axion. This confirms that in the $\frac{1}{2} \otimes \frac{1}{2}$ double copy setup the spin-1 field does not source the axion. Second and more importantly, there is no need for adding a contact interaction between matter lines, a feature we will confirm also in $M_{5}$. This is the only gravitational theory we have found for which this happens and reflects its underlying fermionic origin. 


\subsection{Inelastic scattering}

Moving on to the inelastic scattering, we consider the emission of a gluon or a (fat) graviton in the final state. The relevance of this amplitude is that it allows us to make contact with classical double copy for radiation at the end of this section.

The QCD amplitude obtained from Feynman diagrams can be arranged into the color decomposition (4.1) with only five terms. The color factors and numerators will satisfy

$$
\begin{gathered}
c_{1}-c_{2}=-c_{3}, \quad c_{4}-c_{5}=c_{3}, \\
n_{1}-n_{2}=-n_{3}, \quad n_{4}-n_{5}=n_{3} .
\end{gathered}
$$

The gravitational amplitude will be given again by (4.4), with the sum running from 1 to 5 . The product of polarization vectors of the external gluon $\epsilon_{\mu} \tilde{\epsilon}_{\nu}$ corresponds to a fat graviton state $H_{5}$. To extract the graviton amplitude we replace $\epsilon_{\mu} \tilde{\epsilon}_{\nu} \rightarrow \epsilon_{\mu \nu}^{\mathrm{TT}}$ i.e. the symmetric, transverse and traceless polarization tensor for the graviton. If on the other hand we want to compute the dilaton amplitude, we replace $\epsilon_{\mu} \tilde{\epsilon}_{\nu} \rightarrow \frac{\eta_{\mu \nu}}{\sqrt{D-2}}$. In the same way that for the case of elastic scattering, let us consider different cases for the spin of the massive lines. In the case with spin we will also consider an external axion state with the help of a suitable BCJ gauge.

\subsubsection{Spinless case}

We first review the spinless case as considered in e.g. [20]. The tree-level QCD amplitude (4.1) in BCJ form can be taken from there. We use the momentum conservation condition $k=q_{1}+q_{3}$, to write the numerators, color factors and denominators in (4.1) as follows

$$
\begin{aligned}
& n_{1}^{(0,0)}=e^{3}\left[2\left(2 p_{3}-q_{3}\right) \cdot\left(2 p_{1}+q_{3}\right)\left(p_{1}+q_{3}\right) \cdot \epsilon-\left(2 p_{1} \cdot q_{3}+q_{3}^{2}\right)\left(2 p_{3}-q_{3}\right) \cdot \epsilon\right], \\
& n_{2}^{(0,0)}=e^{3}\left[2 p_{1} \cdot \epsilon\left(2 p_{1}-k-q_{1}\right) \cdot\left(2 p_{3}-q_{3}\right)+2 p_{1} \cdot k\left(2 p_{3}-q_{3}\right) \cdot \epsilon\right], \\
& n_{3}^{(0,0)}=e^{3}\left(2 p_{1}-q_{1}\right)^{\mu}\left(2 p_{3}-q_{3}\right)^{\rho}\left[\left(k+q_{3}\right)_{\mu} \eta_{\nu \rho}+\left(q_{1}-q_{3}\right)_{\nu} \eta_{\mu \rho}-\left(k+q_{1}\right)_{\rho} \eta_{\mu \nu}\right] \epsilon^{\nu}, \\
& n_{4}^{(0,0)}=e^{3}\left[2\left(2 p_{1}-q_{1}\right) \cdot\left(2 p_{3}+q_{1}\right)\left(p_{3}+q_{1}\right) \cdot \epsilon-\left(2 p_{3} \cdot q_{1}+q_{1}^{2}\right)\left(2 p_{1}-q_{1}\right) \cdot \epsilon\right], \\
& n_{5}^{(0,0)}=e^{3}\left[2 p_{3} \cdot \epsilon\left(2 p_{3}-k-q_{3}\right) \cdot\left(2 p_{1}-q_{1}\right)+2 p_{3} \cdot k\left(2 p_{1}-q_{1}\right) \cdot \epsilon\right] . \\
& c_{1}=\left(T_{1}^{a} \cdot T_{1}^{b}\right) T_{3}^{b}, \quad d_{1}=q_{3}^{2}\left(2 p_{1} \cdot k-q_{1}^{2}+q_{3}^{2}\right), \\
& c_{2}=\left(T_{1}^{b} \cdot T_{1}^{a}\right) T_{3}^{b}, \quad d_{2}=-2\left(p_{1} \cdot k\right) q_{3}^{2}, \\
& c_{3}=f^{a b c} T_{1}^{b} T_{3}^{c}, \quad d_{3}=q_{1}^{2} q_{3}^{2}, \\
& c_{4}=\left(T_{3}^{a} \cdot T_{3}^{b}\right) T_{1}^{b}, \quad d_{4}=q_{1}^{2}\left(2 p_{3} \cdot k+q_{1}^{2}-q_{3}^{2}\right), \\
& c_{5}=\left(T_{3}^{b} \cdot T_{3}^{a}\right) T_{1}^{b} . \quad d_{5}=-2\left(p_{3} \cdot k\right) q_{1}^{2} .
\end{aligned}
$$

It is now straightforward to compute the gravitational amplitude $M_{5}^{\mathrm{gr}}$ using (4.4). The comparison of the double copy result with the Feynman diagrammatic computation from the Lagrangian (4.11) shows complete agreement for both the graviton $\left(\epsilon_{\mu} \tilde{\epsilon}_{\nu} \rightarrow \epsilon_{\mu \nu}^{\mathrm{TT}}\right)$ and dilaton $\left(\epsilon_{\mu} \tilde{\epsilon}_{\nu} \rightarrow \frac{\eta_{\mu \nu}}{\sqrt{d-2}}\right)$ amplitude. This agreement provides a non trivial check of the contact interaction we included to match the double copy result for the elastic scattering amplitude. 


\subsubsection{Case $s_{a}=s_{b}=\frac{1}{2}+\frac{1}{2}$}

For the case of the inelastic scattering of two fermions with different flavour, and the emission of one gluon, the QCD amplitude in the BCJ form (4.1) can be computed directly from Feynman diagrams. As in the scalar case the numerators also take a compact form

$$
\begin{aligned}
& n_{1}^{\left(\frac{1}{2}, \frac{1}{2}\right)}=e^{3} \bar{u}_{2} \notin\left(\not p 1+q / 3+m_{a}\right) \gamma^{\mu} u_{1} \bar{u}_{4} \gamma_{\mu} u_{3}, \\
& n_{2}^{\left(\frac{1}{2}, \frac{1}{2}\right)}=e^{3} \bar{u}_{2} \gamma^{\mu}\left(\not p_{1}-\not k+m_{a}\right) \notin u_{1} \bar{u}_{4} \gamma_{\mu} u_{3}, \\
& n_{4}^{\left(\frac{1}{2}, \frac{1}{2}\right)}=e^{3} \bar{u}_{2} \gamma^{\mu} u_{1} \bar{u}_{4} \notin\left(\not p_{3}+q 1 / m_{b}\right) \gamma_{\mu} u_{3}, \\
& n_{5}^{\left(\frac{1}{2}, \frac{1}{2}\right)}=e^{3} \bar{u}_{2} \gamma^{\mu} u_{1} \bar{u}_{4} \gamma^{\mu}\left(\not p_{3}-\not k+m_{b}\right) \notin u_{3}, \\
& n_{3}^{\left(\frac{1}{2}, \frac{1}{2}\right)}=-2 e^{3}\left(\bar{u}_{2} \notin u_{1} \bar{u}_{4} \not k u_{3}-\bar{u}_{2} \not k u_{1} \bar{u}_{4} \notin u_{3}-\bar{u}_{2} \gamma^{\mu} u_{1} \bar{u}_{4} \gamma_{\mu} u_{3} q_{1} \cdot \epsilon\right) .
\end{aligned}
$$

Analogously, their charge conjugated pairs read

$$
\begin{aligned}
& \bar{n}_{1}^{\left(\frac{1}{2}, \frac{1}{2}\right)}=e^{3} \bar{v}_{1} \gamma^{\mu}\left(\not p_{1}+q p_{3}-m_{a}\right) \tilde{\phi}_{2} \bar{v}_{3} \gamma_{\mu} v_{4}, \\
& \bar{n}_{2}^{\left(\frac{1}{2}, \frac{1}{2}\right)}=e^{3} \bar{v}_{1} \tilde{\phi}\left(\not p_{1}-\not k-m_{a}\right) \gamma^{\mu} v_{2} \bar{v}_{3} \gamma_{\mu} v_{4}, \\
& \bar{n}_{4}^{\left(\frac{1}{2}, \frac{1}{2}\right)}=e^{3} \bar{v}_{1} \gamma^{\mu} v_{2} \bar{v}_{3} \gamma_{\mu}\left(\not p_{3}+q \not 1-m_{b}\right) \tilde{\phi} v_{4}, \\
& \bar{n}_{5}^{\left(\frac{1}{2}, \frac{1}{2}\right)}=e^{3} \bar{v}_{1} \gamma^{\mu} v_{2} \bar{v}_{3} \tilde{\phi}\left(\not p_{3}-\not k-m_{b}\right) \gamma^{\mu} v_{4}, \\
& \bar{n}_{3}^{\left(\frac{1}{2}, \frac{1}{2}\right)}=-2 e^{3}\left(\bar{v}_{1} \tilde{\phi}_{2} \bar{v}_{3} \not k v_{4}-\bar{v}_{1} \not k v_{2} \bar{v}_{3} \tilde{\phi}_{4}-\bar{v}_{1} \gamma^{\mu} v_{2} \bar{v}_{3} \gamma_{\mu} v_{4} q_{1} \cdot \tilde{\epsilon}\right) .
\end{aligned}
$$

The color factors and denominators are the same as the scalar case (4.25). The spin$\frac{1}{2}$ numerators are readily seen to satisfy the kinematic Jacobi relation (4.23). Now, to compute the gravitational amplitude (4.4), we need to compute the double copy of these numerators using (4.7). For instance we have

$$
\begin{aligned}
n_{1}^{\left(\frac{1}{2}, \frac{1}{2}\right)} \otimes \bar{n}_{1}^{\left(\frac{1}{2}, \frac{1}{2}\right)}= & \frac{\kappa^{3}}{2^{2\lfloor d / 2\rfloor+4}} \operatorname{tr}\left[\notin\left(\not p 1+\not \beta_{3}+m_{a}\right) \gamma^{\mu} \ddagger_{1}\left(\not p_{1}-m_{a}\right) \gamma^{\nu}\left(\not p 1+\not \beta_{3}-m_{a}\right) \tilde{\phi}_{2}\left(\not p_{2}+m_{a}\right)\right] \\
& \times \operatorname{tr}\left[\gamma_{\mu} \ddagger_{3}\left(\not p_{3}-m_{b}\right) \gamma_{\nu} \ddagger_{4}\left(\not p_{4}+m_{b}\right)\right] .
\end{aligned}
$$

Analogous expressions follow for the double copy of the remaining numerators. Although this is a very compact way to write the gravitational numerator, the final result for the gravitational amplitude (4.4) after doing all the traces is too long to be printed in this paper, therefore we provide a Mathematica Notebook with the result.

As we did for the case of elastic scattering, we have checked that the same amplitude can be computed with the Feynman rules derived from the Lagrangian (4.21), for both graviton and dilaton radiation. This is in fact a non-trivial check of the Lagrangian, and discards the need for a quartic term, or the presence of the axion field.

Finally, we leave the discussion for the case $s_{a}=0+1$ and $s_{b}=0+0$, and the case $s_{a}=s_{b}=0+1$, to be illustrated in section 4.3. Using a more convenient BCJ gauge, these amplitudes take compact expressions and the numerator can be put in a manifestly gauge invariant form. 


\subsection{Generalized gauge transformations and classical radiation}

Now that we have computed the amplitude for different Bremsstrahlung processes we might ask what sort of classical information can be extracted from it. The answer is given by the radiated momentum which is carried by long range fields (photons, gravitons, dilatons and axions) to null infinity $[22,78]$. This momentum is determined by a phase space integral,

$$
K^{\mu}=\int \mathrm{dLIPS}(k) k^{\mu}|J(k)|^{2},
$$

where $J(k)$ is the radiative piece of the stress energy tensor (or current) related to the amplitude via the LSZ formula. This also requires to implement a prescription for the classical limit, $J(k)=\lim _{\hbar \rightarrow 0} M_{5}[20,78]$. In light of the promising recent developments of $[21,25,28,31]$ it is desirable to understand how a double copy structure turns out to be realized in classical radiation, and more specifically, how it follows from the BCJ construction in QFT.

We would like to extract the classical piece of the amplitude in such a way that the double copy structure is preserved untouched in the final result. Taking the classical limit of (4.4) however does not show explicitly the double copy form of the classical amplitude as we will see in a moment. This was first observed for scalar sources in [20], but is also true for the spinning case. We find that the problem can be fixed if we write the double copy for inelastic scattering in a more convenient generalized gauge. This further provides an alternative derivation to the classical double copy formula presented in [47].

\subsubsection{Classical radiation from the standard BCJ double copy}

For scalar radiation the comparison of the classical construction derived by Goldberger and Ridgway [22] and the classical limit of the BCJ double copy result was presented in [20]. There the limit was taken by means of a large mass expansion. Here we follow will [78] instead, where it was shown that the classical piece of the amplitude can be obtained by re-scaling the massless transfer momenta with $\hbar$ and take the leading order piece in the $\hbar \rightarrow 0$ limit. With this in mind it is convenient for us to introduce the average momentum transfer $q=\frac{q_{1}-q_{3}}{2}$. The re-scaled momenta can be interpreted as a classical wave vector $q \rightarrow \hbar \bar{q}$. Notice that momentum conservation implies that the radiated on-shell momenta needs to be re-scaled as well $k \rightarrow \hbar \bar{k}$. For spinning radiation the classical limit was outlined in [47] and requires to introduce the angular momentum operator, performing the multipole expansion as we have described in the previous sections. We then scale such operator as $J \rightarrow \hbar^{-1} \bar{J}[47,50]$ and strip the respective polarization states [52], see [107] for a formal derivation. Finally, for the case of QCD amplitudes, one further scaling needs to be done in order to correctly extract the classical piece. In reminiscence of the color-kinematics duality, we find that the generators of the color group $T^{a}$ must also scale as those of angular momentum, i.e. $T^{a} \rightarrow \hbar^{-1} T^{a}$.

In order to motivate our procedure let us first consider the 5-pt. amplitudes for both QCD and gravity in the standard BCJ form we have provided. In other words, we want to see how the ingredients in (4.1) and (4.4) behave in the $\hbar$-expansion. By inspection, the leading order of the numerators $n_{i}$ goes as $\hbar^{0}$, and the sub-leading correction is of order $\hbar$. 
Let us denote the expansion of the numerators as $n_{i}=\left\langle n_{i}\right\rangle+\delta n_{i} \hbar+\cdots$. The denominators can also be expanded as $d_{i}=\left\langle d_{i}\right\rangle \hbar^{3}+\delta d_{i} \hbar^{4}+\cdots$. At leading order, it is easy to check that $\left\langle n_{3}\right\rangle=0,\left\langle n_{1}\right\rangle=\left\langle n_{2}\right\rangle$ and $\left\langle n_{4}\right\rangle=\left\langle n_{5}\right\rangle$, whereas for the denominators we have $\left\langle d_{1}\right\rangle=-\left\langle d_{2}\right\rangle$ and $\left\langle d_{4}\right\rangle=-\left\langle d_{5}\right\rangle$. At sub-leading order $\delta d_{2}=\delta d_{5}=0$. Finally, for the color factors we have $c_{i} \rightarrow \hbar^{-3} c_{i}$ for $i=1,2,4,5$ and $c_{3} \rightarrow \hbar^{-2} c_{3}$.

With this in mind, the classical piece of the QCD amplitude for gluon radiation reads

$$
\left\langle M_{5}^{\mathrm{QCD}}\right\rangle=-c_{1}\left[\frac{\left\langle n_{1}\right\rangle \delta d_{1}}{\left\langle d_{1}\right\rangle^{2}}-\frac{\delta n_{1}-\delta n_{2}}{\left\langle d_{1}\right\rangle}\right]-c_{3}\left[\frac{\left\langle n_{1}\right\rangle}{\left\langle d_{1}\right\rangle}-\frac{\delta n_{3}}{\delta d_{3}}-\frac{\left\langle n_{4}\right\rangle}{\left\langle d_{4}\right\rangle}\right]-c_{4}\left[\frac{\left\langle n_{4}\right\rangle \delta d_{4}}{\left\langle d_{4}\right\rangle^{2}}-\frac{\delta n_{4}-\delta n_{5}}{\left\langle d_{4}\right\rangle}\right],
$$

where $\left\langle M_{n}\right\rangle:=\lim _{\hbar \rightarrow 0} M_{n}$. A similar expansion can be done for the gravitational amplitude given by the double copy (4.4)

$$
\begin{aligned}
\left\langle M_{5}^{g r}\right\rangle= & -\frac{\left\langle n_{1}\right\rangle \otimes\left\langle\tilde{n}_{1}\right\rangle}{\left\langle d_{1,0}\right\rangle^{2}} \delta d_{1}+\frac{\left\langle n_{1}\right\rangle \otimes\left(\delta \tilde{n}_{1}-\delta \tilde{n}_{2}\right)+\left(\delta n_{1}-\delta n_{2}\right) \otimes\left\langle\tilde{n}_{1}\right\rangle}{\left\langle d_{1}\right\rangle}+\frac{\delta n_{3} \otimes \delta \tilde{n}_{3}}{\left\langle d_{3}\right\rangle} \\
& -\frac{\left\langle n_{4}\right\rangle \otimes\left\langle\tilde{n}_{4}\right\rangle}{\left\langle d_{4,0}\right\rangle^{2}} \delta d_{4}+\frac{\left\langle n_{4}\right\rangle \otimes\left(\delta \tilde{n}_{4}-\delta \tilde{n}_{5}\right)+\left(\delta n_{4}-\delta n_{5}\right) \otimes\left\langle\tilde{n}_{4}\right\rangle}{\left\langle d_{4}\right\rangle}
\end{aligned}
$$

Hence, we find that the classical piece of the gravitational amplitude (4.31) does not reflect the BCJ double copy structure as expected. This can be traced back to the presence of $\frac{1}{\hbar}$ terms which will still contribute to the expansion even though the overall leading order (as $\hbar \rightarrow 0$ ) cancels. We shall find a way to make such limit smooth and preserve the double copy structure.

In [47] we provided a classical double copy formula to compute gravitational radiation at leading order in the coupling from photon Bremsstrahlung. The formula was obtained by looking at specific cuts carrying the classical information. Here we will see it follows naturally from a particular BCJ gauge. In fact, in such gauge we will further find no $\frac{1}{\hbar}$ terms and hence a smooth classical limit.

Before we start let us summarize the results. The classical expressions for gluon and "fat graviton" radiation (4.30) and (4.31) agrees with the result of Goldberger and Ridgway for scalar sources [22], as shown in [20]. Here we will further recover the results of Goldberger, Li and Prabhu [26, 27] for spinning sources up to dipole order, including the full axion-dilaton-graviton classical radiation. We also make contact with our own results in [47] which already recovered graviton radiation in such case. This will be achieved by providing the classical numerators for each of these cases in the aforementioned gauge.

\subsubsection{Generalized gauge transformation}

In order to rewrite the quantum amplitudes (4.1) and (4.4) in a convenient gauge we proceed as follows. Observe that the non-abelian contribution to the QCD amplitude (4.1) comes from the diagram whose color factor (4.25) is $c_{3}$, which is proportional to the structure constants of the gauge group. We can however gauge away this non-abelian piece of the amplitude using a Generalized Gauge Transformation (GGT) [1]. Recall that a GGT is a transformation on the kinematic numerators that leaves the amplitude invariant. This transformation allow us to move terms between diagrams. For the case of the inelastic 
scattering, consider the following shift on the numerators entering in (4.1)

$$
\begin{aligned}
& n_{1}^{\prime}=n_{1}-\alpha d_{1}, \\
& n_{2}^{\prime}=n_{2}+\alpha d_{2}, \\
& n_{3}^{\prime}=n_{3}-\alpha d_{3}+\gamma d_{3}, \\
& n_{4}^{\prime}=n_{4}-\gamma d_{4}, \\
& n_{5}^{\prime}=n_{5}+\gamma d_{5} .
\end{aligned}
$$

This shift leaves invariant the amplitude (4.1) since

$$
\Delta M_{5}^{\mathrm{QCD}}=-\alpha\left(c_{1}-c_{2}+c_{3}\right)-\gamma\left(c_{4}-c_{5}-c_{3}\right)=0,
$$

where we have use the color identities (4.22) in the last equality. We can now solve for the values of $\alpha$ and $\gamma$ that allow to impose $n_{3}^{\prime}=0$, while satisfying the color-kinematic duality for the shifted numerators

$$
n_{1}^{\prime}-n_{2}^{\prime}=-n_{3}^{\prime}=0, \quad n_{4}^{\prime}-n_{5}^{\prime}=n_{3}^{\prime}=0 .
$$

The solution can be written as

$$
\alpha=-\frac{n_{3}}{d_{1}+d_{2}}, \quad \gamma=-\frac{d_{1}+d_{2}+d_{3}}{d_{1}+d_{2}} \frac{n_{3}}{d_{3}} .
$$

Explicitly these parameters take the simple form

$$
\alpha=\frac{n_{3}}{2 q \cdot k\left(q^{2}-q \cdot k\right)}, \quad \gamma=\frac{n_{3}}{2 q \cdot k\left(q^{2}+q \cdot k\right)},
$$

Importantly, this solution is general and independent of the spin of scattered particles.

The new numerators (4.32) will be non-local since they have absorbed $n_{3}$. However, they exhibit nice features: they are independent, gauge invariant, and in the classical limit they will lead to a remarkably simple (and local!) form. Indeed, the QCD amplitude (4.1) for inelastic scattering takes already a more compact form

$$
M_{5}^{\mathrm{QCD}}=\left[\frac{c_{1}}{d_{1}}+\frac{c_{2}}{d_{2}}\right] n_{1}^{\prime}+\left[\frac{c_{4}}{d_{4}}+\frac{c_{5}}{d_{5}}\right] n_{4}^{\prime}
$$

The gravitational amplitude (4.4) then is given by the double copy of (4.37) as follows

$$
M_{5}^{\mathrm{gr}}=\frac{n_{1}^{\prime} \otimes \tilde{n}_{1}^{\prime}}{d_{1}^{\prime}}+\frac{n_{4}^{\prime} \otimes \tilde{n}_{4}^{\prime}}{d_{4}^{\prime}},
$$

where

$$
d_{1}^{\prime}=\frac{d_{1} d_{2}}{d_{1}+d_{2}}, \quad d_{4}^{\prime}=\frac{d_{4} d_{5}}{d_{4}+d_{5}}
$$

Explicitly, this gives

$$
\frac{1}{d_{1}^{\prime}}=-\frac{q \cdot k}{p_{1} \cdot k q \cdot(q-k)\left(2 q \cdot k-2 p_{1} \cdot k\right)}, \quad \frac{1}{d_{4}^{\prime}}=-\frac{q \cdot k}{p_{3} \cdot k q \cdot(q+k)\left(2 q \cdot k+2 p_{3} \cdot k\right)},
$$


When performing the double copy, there will in principle be a pole in $q \cdot k$ both in (4.38) and in the classical formula (4.42) below, which is nevertheless spurious and cancels out in the final result. Notice we have reduced the problem of doing the double copy of five numerators to do the double copy of just two (the dimension of the BCJ basis). Indeed, now we can take $c_{3} \rightarrow 0$, setting $c_{2} \rightarrow c_{1}$ and $c_{5} \rightarrow c_{4}$. Further fixing $c_{1}=c_{4}=1$ we obtain the QED case (see (4.25)) with

$$
M_{5}^{\mathrm{QED}}=\frac{n_{1}^{\prime}}{d_{1}^{\prime}}+\frac{n_{4}^{\prime}}{d_{4}^{\prime}}
$$

The double copy formula (4.38) agrees with (4.4). Remarkably, we can use (4.41) as a starting point for the (classical) double copy since the numerators $n_{1}^{\prime}$ and $n_{4}^{\prime}$ can be read off from $M_{5}^{\mathrm{QED}}$ from its pole structure. This has the advantage that the classical limit of the amplitude will be smooth and will preserve the double copy form.

\subsubsection{Classical limit and Compton residue}

In the gauge (4.32), extracting the classical piece of the gravitational amplitude (4.38) is straightforward. The shifted numerators scale as $n_{i}^{\prime}=\left\langle n_{i}^{\prime}\right\rangle+\delta n_{i}^{\prime} \hbar$, whereas the denominators scale as $d_{i}^{\prime}=\left\langle d_{i}^{\prime}\right\rangle \hbar^{2}+\delta d_{i}^{\prime} \hbar^{3}$. With this in mind, the classical piece of the gravitational amplitude (4.38) is simply

$$
\left\langle M_{5}^{\left(s_{a} \otimes \tilde{s}_{a}, s_{b} \otimes \tilde{s}_{b}\right)}\right\rangle=\frac{\left\langle n_{1}^{\prime\left(s_{a}, s_{b}\right)}\right\rangle \otimes\left\langle\tilde{n}_{1}^{\prime\left(\tilde{s}_{a}, \tilde{s}_{b}\right)}\right\rangle}{\left\langle d_{1}^{\prime}\right\rangle}+\frac{\left\langle n_{4}^{\prime\left(s_{a}, s_{b}\right)}\right\rangle \otimes\left\langle\tilde{n}_{4}^{\prime\left(\tilde{s}_{a}, \tilde{s}_{b}\right)}\right\rangle}{\left\langle d_{4}^{\prime}\right\rangle},
$$

which shows explicitly the double copy structure. Indeed, the classical limit of the QED amplitude is naturally identified as the single copy in this gauge:

$$
\left\langle M_{5}^{\mathrm{QED},\left(s_{a}, s_{b}\right)}\right\rangle=\frac{\left\langle n_{1}^{\prime\left(s_{a}, s_{b}\right)}\right\rangle}{\left\langle d_{1}^{\prime}\right\rangle}+\frac{\left\langle n_{4}^{\prime\left(s_{a}, s_{b}\right)}\right\rangle}{\left\langle d_{4}^{\prime}\right\rangle} .
$$

Taking the classical piece of the denominators (4.40) leads to

$$
\frac{1}{\left\langle d_{1}^{\prime}\right\rangle}=\frac{q \cdot k}{2\left(p_{1} \cdot k\right)^{2}\left(q^{2}-q \cdot k\right)}, \quad \frac{1}{\left\langle d_{4}^{\prime}\right\rangle}=-\frac{q \cdot k}{2\left(p_{3} \cdot k\right)^{2}\left(q^{2}+q \cdot k\right)} .
$$

As a whole, the formulas (4.42), (4.43) and (4.44) correspond to the construction given in [47]. The conversion can be done via $\left\langle n_{i}^{\prime}\right\rangle=\frac{2}{q \cdot k} n_{i}^{\text {there }}$, where $n_{i}^{\text {there }}$ is a local numerator in the classical limit. We have thus found here an alternative derivation which follows directly from the standard BCJ double copy of $M_{5}$, up to certain details we now describe.

Suppose first that the numerators $\left\langle n_{i}^{\prime}\right\rangle$ do not depend on $q^{2}$. Then we find they can be read off from the QED Compton residues at $q^{2} \rightarrow \pm q \cdot k$. Indeed, using that (4.43)-(4.44) should factor into the Compton amplitude $A_{4}$ together with a 3 -pt. amplitude $A_{3}$, we get

$$
\left\langle n_{i}^{\prime\left(s_{a}, s_{b}\right)}\right\rangle=\frac{2(p \cdot k)^{2}}{q \cdot k}\left\langle A_{4}^{\mathrm{QED}, s_{a}, \mu}\right\rangle \times\left\langle A_{3}^{\mathrm{QED}, s_{b}, \mu},\right\rangle
$$


where the contraction in $\mu$ denotes propagation of photons. This guarantees the same is true for the gravitational numerators in (4.42), that is

$$
\begin{aligned}
\left\langle n_{i}^{\prime\left(s_{a}, s_{b}\right)}\right\rangle \otimes\left\langle n_{i}^{\prime\left(s_{a}, s_{b}\right)}\right\rangle & =\frac{4(p \cdot k)^{4}}{(q \cdot k)^{2}}\left\langle A_{4}^{\mathrm{QED}, s_{a}, \mu}\right\rangle \otimes\left\langle A_{4}^{\mathrm{QED}, \tilde{s}_{a}, \nu}\right\rangle \times\left\langle A_{3}^{\mathrm{QED}, s_{b}, \mu}\right\rangle \otimes\left\langle A_{3}^{\mathrm{QED}, \tilde{s}_{b}, \nu}\right\rangle, \\
& =\frac{4(p \cdot k)^{4}}{(q \cdot k)^{2}}\left\langle A_{4}^{s_{a} \otimes \tilde{s}_{a}, \mu \nu}\right\rangle \times\left\langle A_{3}^{s_{b} \otimes \tilde{s}_{b}, \mu \nu}\right\rangle,
\end{aligned}
$$

where the contracted indices denote propagation of fat states. Thus we conclude that the classical limit is controlled by $A_{4}$ and $A_{3}$ via the Compton residues provided the numerators do not depend on $q^{2}$. Considering the scaling of the multipoles $J \rightarrow \hbar^{-1} \bar{J}$ and that $q \rightarrow \hbar \bar{q}$, we see that this is true up to dipole $\sim J$ order. We will confirm this explicitly in the cases below.

At quadrupole order $\sim J^{2}$, associated to spin-1 particles, we will find explicit dependence on $q^{2}$ in the numerators. Nevertheless, it is still true that the classical multipoles are given by the Compton residues as we have exemplified already in [47]. Indeed, as a quick analysis shows, the $q^{2}$ dependence in $M_{5}$ that is not captured by them can only arise from 1) contact terms in $M_{5}$ or 2) contact terms in $M_{4}$ entering through the residues at $p \cdot k \rightarrow 0$. Both contributions can be canceled by adding appropriate (quantum) interactions between the matter particles. Note that canceling such contributions in the QCD side will automatically imply their cancellation on the gravity side.

Let us now see some specific examples of how to write the amplitudes (4.38) and their classical pieces (4.42)-(4.43), in the gauge (4.32).

\subsubsection{Spinless case}

In the gauge (4.32), the scalar numerators take an explicit gauge invariant form

$$
\begin{aligned}
& n_{1}^{\prime(0,0)}=e^{3} \frac{8 p_{1} \cdot k\left(p_{1} \cdot F \cdot p_{3}-q \cdot F \cdot p_{3}\right)+2\left(4 p_{3} \cdot k-4 p_{1} \cdot p_{3}-q \cdot(q-k)\right) q \cdot F \cdot p_{1}}{q \cdot k} \\
& n_{4}^{\prime(0,0)}=e^{3} \frac{8 p_{3} \cdot k\left(p_{1} \cdot F \cdot p_{3}-q \cdot F \cdot p_{1}\right)+2\left(4 p_{1} \cdot k-4 p_{1} \cdot p_{3}-q \cdot(q+k)\right) q \cdot F \cdot p_{3}}{q \cdot k} .
\end{aligned}
$$

Observe these numerators contain $q^{2}$ dependence. Nevertheless it is completely quantum as the only classical piece is the leading order in $q$, given by

$$
\left\langle n_{1}^{\prime(0,0)}\right\rangle=\frac{8 e^{3}}{q \cdot k} p_{1} \cdot R \cdot F \cdot p_{1}, \quad\left\langle n_{4}^{\prime(0,0)}\right\rangle=\frac{8 e^{3}}{q \cdot k} p_{3} \cdot R_{1} \cdot F \cdot p_{3},
$$

where $R_{i}^{\mu \nu}=p_{i}^{[\mu}\left(\eta_{i} 2 q-k\right)^{\nu]}$, and $\eta_{1}=-1, \eta_{3}=1$. It is very easy to check that these numerators reproduce the classical photon radiation of [22] and in fact can be read from there by looking at the pole structure. The classical "fat graviton" radiation (4.42) for scalar sources can be computed from the double copy of the classical scalar numerators (4.49) with themselves. It can be shown that these results agree with [22]. Here however we have taken advantage of the GGT to keep the double copy structure of the classical gravitational amplitude. 


\subsubsection{Case $s_{a}=0+1$ and $s_{b}=0+0$}

Now that we have understood how to compute classical radiation for scalars, we can add spin to particle $a$, whereas particles $b$ remains spinless. The gravitational amplitude $M_{5}^{(0 \otimes 1,0 \otimes 0)}$ computed from (4.38) can be computed from the double copy of the spinless numerators (4.47)-(4.48) with the following numerators:

$$
\begin{aligned}
n_{1}^{\prime(0,1)}=\frac{2 e^{3}}{q \cdot k}\{[ & \left.\left(q^{2}-q \cdot k+4 p_{1} \cdot p_{3}\right) q \cdot F \cdot p_{1}+4\left(q-p_{1}\right) \cdot k p_{1} \cdot F \cdot p_{3}\right] \varepsilon_{1} \cdot \varepsilon_{2} \\
& -\left[4\left(p_{1} \cdot k p_{3 \mu} F_{\alpha \nu} q^{\alpha}-q \cdot k p_{3 \mu} F_{\nu \alpha} p_{1}^{\alpha}\right)+8\left(p_{1} \cdot k q_{\mu} F_{\nu \alpha} p_{3}^{\alpha}-q_{\mu} p_{3 \nu} q \cdot F \cdot p_{1}\right)\right. \\
& \left.-2\left(2 p_{3} \cdot k\left(p_{1}-q\right) \cdot k+q \cdot k\left(q^{2}-q \cdot k+4 p_{1} \cdot p_{3}\right)\right) F_{\mu \nu}\right] \varepsilon_{1}^{[\mu} \varepsilon_{2}^{\nu]} \\
& \left.-2 q \cdot k\left(4 q^{\alpha} F_{\alpha \mu} p_{3 \nu}-4 p_{3}^{\alpha} F_{\alpha \mu} q_{\nu}+2 k_{\mu} p_{3}^{\alpha} F_{\alpha \nu}\right) \varepsilon_{1}^{\mu} \varepsilon_{2}^{\nu}\right\} \\
n_{4}^{\prime(0,1)}=\frac{2 e^{3}}{q \cdot k}\{[ & \left.\left(q^{2}+q \cdot k+4 p_{1} \cdot p_{3}\right) q \cdot F \cdot p_{3}-4\left(q+p_{3}\right) \cdot k p_{1} \cdot F \cdot p_{3}\right] \varepsilon_{1} \cdot \varepsilon_{2} \\
& \left.+\left[2\left(q+p_{3}\right) \cdot k\left(4 p_{3}^{\alpha} q_{\mu} F_{\alpha \nu}+p_{3} \cdot k F_{\mu \nu}\right)+4 p_{3 \mu}(2 q+k)_{\nu}\right] \varepsilon_{1}^{[\mu} \varepsilon_{2}^{\nu]}\right\}
\end{aligned}
$$

These numerators can be obtained from the QCD action (3.19), by considering a $W$ boson interacting with a scalar particle through gluons, and then applying the GGT (4.32). Alternatively, they correspond to the QED theory as already explained.

The amplitude $M_{5}^{(0 \otimes 1,0 \otimes 0)}$ is in complete agreement with the Feynman diagrammatic computation from the Lagrangian (4.13), which as in the case of scalar sources, provides a strong check of the contact interaction introduced to match the double copy for the elastic scattering.

Now we can take the classical limit of numerators (4.50) and (4.51). To make contact with the multipole expansion we write the results in terms of the Lorentz generator $J_{1}^{\mu \nu}$ acting on a spin-1 representation. We strip the matter polarization vectors for simplicity and keep the operators. Up to dipole order, the operators are given by

$$
\begin{aligned}
& \left\langle n_{1}^{\prime(0,1)}\right\rangle=\left\langle n_{1}^{\prime(0,0)}\right\rangle-\frac{4 e^{3}}{q \cdot k}\left[p_{1} \cdot R_{3} \cdot k F \cdot J_{1}-F_{1 q} R_{3} \cdot J_{1}+p_{1} \cdot k\left[F, R_{3}\right] \cdot J_{1}\right], \\
& \left\langle n_{4}^{\prime(0,1)}\right\rangle=\left\langle n_{4}^{\prime(0,0)}\right\rangle+\frac{4 e^{3}}{q \cdot k} p_{3} \cdot F \cdot \hat{R}_{1} \cdot p_{3},
\end{aligned}
$$

where we have used $F_{i q}=\eta_{i}\left(p_{i} \cdot F \cdot q\right)$, and $\hat{R}_{i}=\left(\eta_{i} 2 q-k\right)^{[\mu} J_{i}^{\nu] \alpha}\left(\eta_{i} 2 q-k\right)_{\alpha}$. Also $[F, R]_{\mu \nu}=$ $F_{\mu \alpha} R_{\nu}^{\alpha}-(\mu \leftrightarrow \nu)$, etc. These classical numerators agree with the ones given in [47]. Note that the $q^{2}$ dependence in (4.50), (4.51) has become has become suppressed by powers of $J$. In [47] we have nevertheless managed to cancel it by adding flavor interactions, hence obtaining also the form of the classical gravitational quadrupole $\sim J^{2}$.

Finally, the QED and gravitational radiation constructed from (4.52), (4.53) agrees with the results of $\mathrm{Li}$ and Prahbu [27] when we set one of the massive objects to be spinless. 


\subsubsection{Case $s_{a}=s_{b}=0+1$}

We can now move on to the case in which both massive lines have spin- 1 in the gravitational theory. We want to compute the gravitational amplitude for inelastic scattering $M_{5}^{(0 \otimes 1,0 \otimes 1)}$ using (4.38). The scalar numerators are given in (4.47) and (4.48). The numerators for the spining case are constructed following the considerations of section 4.1 .3 and give

$$
\begin{aligned}
n_{1}^{\prime(1,1)}= & \frac{2 e^{3}}{q \cdot k}\left\{\left[\left(q^{2}-q \cdot k+4 p_{1} \cdot p_{3}\right) q \cdot F \cdot p_{1}+4\left(q-p_{1}\right) \cdot k p_{1} \cdot F \cdot p_{3}\right] \varepsilon_{1} \cdot \varepsilon_{2} \varepsilon_{3} \cdot \varepsilon_{4}\right. \\
& +\left[8\left(q-p_{1}\right) \cdot k q \cdot \epsilon_{2} q_{\mu} \epsilon_{1}^{\alpha} F_{\alpha \nu}+4\left[q \cdot k\left(2 p_{1 \mu} q_{\nu}+\left(q-p_{1}\right)_{\mu} k_{\nu}\right)+p_{1} \cdot k k_{\mu} q_{\nu}\right] \varepsilon_{1} \cdot F \cdot \varepsilon_{2}\right. \\
& +4 q_{\mu}\left(2 q \cdot \varepsilon_{1} p_{1} \cdot k-k \cdot \varepsilon_{1} q \cdot k\right) \varepsilon_{2}^{\alpha} F_{\alpha \nu}-\left[4 p_{1} \cdot k q_{\alpha} k_{\beta} \varepsilon_{1}^{[\alpha} \varepsilon_{2}^{\beta]}+q \cdot k k \cdot \varepsilon_{1}(2 q-k) \cdot \varepsilon_{2}\right] F_{\mu \nu} \\
& +\left[2\left(q-p_{1}\right) \cdot k\left(4 q_{\mu} p_{1}^{\alpha} F_{\nu \alpha}+p_{1} \cdot k F_{\mu \nu}\right)+4 p_{1 \mu}(2 q-k)_{\nu} q \cdot F \cdot p_{1}\right] \varepsilon_{1} \cdot \varepsilon_{2} \\
& \left.-4\left(p_{1} \cdot k q_{\rho} F^{\rho \sigma}+q \cdot k p_{1 \rho} F^{\rho \sigma}+2 q^{\sigma} q \cdot F \cdot p_{1}\right) \varepsilon_{1[\mu} \varepsilon_{2 \sigma]}(2 q-k)_{\nu}-4 q \cdot k q \cdot F \cdot \varepsilon_{1} \varepsilon_{2 \mu}(2 q-k)_{\nu}\right] \\
& \times \varepsilon_{3}^{[\mu} \varepsilon_{4}^{\nu]}+\left[4 q \cdot \varepsilon_{2}\left(q-p_{1}\right) \cdot k p_{3} \cdot F \cdot \varepsilon_{1}+2\left(2 q \cdot \varepsilon_{1} p_{1} \cdot k-q \cdot k k \cdot \varepsilon_{1}\right) p_{3} \cdot F \cdot \varepsilon_{2}\right. \\
& -8 p_{3 \mu} q_{\nu} q \cdot F \cdot p_{1} \varepsilon_{1}^{[\mu} \varepsilon_{2}^{\nu]}-2 p_{1} \cdot k p_{3} \cdot \varepsilon_{1} q \cdot F \cdot \varepsilon_{2}-4 q \cdot k p_{3 \mu} p_{1}^{\alpha} F_{\alpha \nu} \varepsilon_{1}^{[\mu} \varepsilon_{2}^{\nu]} \\
& \left.\left.-2\left(2 q-p_{1}\right) \cdot k p_{3} \cdot \varepsilon_{2} q \cdot F \cdot \varepsilon_{1}+\left(q \cdot k\left(q^{2}-q \cdot k+4 p_{1} \cdot p_{3}\right)-2\left(q-p_{1}\right) \cdot k p_{3} \cdot k\right) \varepsilon_{1} \cdot F \cdot \varepsilon_{2}\right] \varepsilon_{3} \cdot \varepsilon_{4}\right\} .
\end{aligned}
$$

The numerator $n_{4}^{\prime(1,1)}$ is given by exchanging particles $a \leftrightarrow b$ in $n_{1}^{\prime(1,1)}$, with $q \rightarrow-q$. The result expressed in terms of these numerators is far more compact than the Feynman diagram expansion obtained from the covariantized Lagrangian (4.15).

Now, by taking the classical limit of the numerators (4.54) we can compute the amplitude $\left\langle M_{5}^{(0 \otimes 1,0 \otimes 1)}\right\rangle$ via (4.42), using also (4.49) and (4.44). In the multipole form of the previous section, the numerators read, up to dipole order,

$$
\begin{aligned}
& \left\langle n_{1}^{\prime(1,1)}\right\rangle=\left\langle n_{1}^{\prime(0,0)}\right\rangle-4 e^{3}\left[p_{1} \cdot R_{3} \cdot k F \cdot J_{1}-F_{1 q} R_{3} \cdot J_{1}+p_{1} \cdot k\left[F, R_{3}\right] \cdot J_{1}-p_{1} \cdot F \cdot \hat{R}_{3} \cdot p_{1}\right] \\
& \left\langle n_{4}^{\prime(1,1)}\right\rangle=\left\langle n_{4}^{\prime(0,0)}\right\rangle-4 e^{3}\left[p_{3} \cdot R_{1} \cdot k F \cdot J_{3}-F_{3 q} R_{1} \cdot J_{3}+p_{3} \cdot k\left[F, R_{1}\right] \cdot J_{3}-p_{3} \cdot F \cdot \hat{R}_{1} \cdot p_{3}\right]
\end{aligned}
$$

Classical radiation computed in this way agrees with the classical double copy result of Goldberger, Li and Prabhu [26, 27] for the whole Fat Graviton radiative field given in eq. (51) of [27] up to dipole order.

Now, we have seen that an alternative Lagrangian construction for the $0 \otimes 1$ double copy at two matter lines is given by the extension of $\mathcal{N}=4$ Supergravity. We know that the

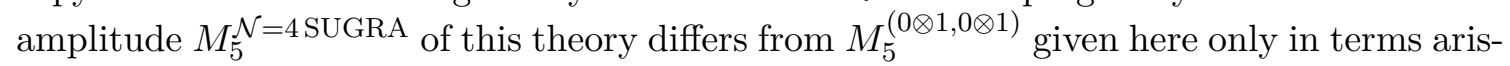
ing from (quantum) flavour interactions, cf. (4.16) vs (3.42). Equivalently both amplitudes have the same residues as $q^{2} \rightarrow \pm q \cdot k$ and hence the same classical limit. As explained, these cuts correspond to Compton amplitudes and in this case feature the propagation of the axion, dilaton and graviton. We have checked these cuts explicitly by comparing the

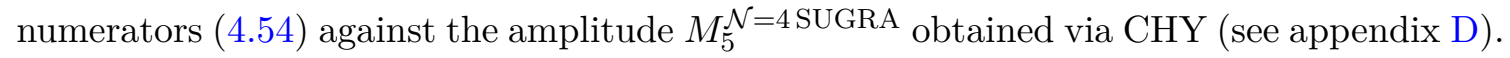




\subsubsection{Case $s_{a}=s_{b}=\frac{1}{2}+\frac{1}{2}$}

The final case for inelastic scattering in the gauge (4.32) is given by the factorization of the gravitational amplitude (4.38) as $s_{a}=s_{b}=\frac{1}{2}+\frac{1}{2}$. For the QCD theory, the shifted numerators entering in (4.37) are

$$
\begin{aligned}
& n_{1}^{\prime\left(\frac{1}{2}, \frac{1}{2}\right)}=\frac{4 e^{3} F_{\alpha \beta}}{q \cdot k}\left[q^{[\alpha} p_{1}^{\beta]} \bar{u}_{2} \gamma^{\mu} u_{1} \bar{u}_{4} \gamma_{\mu} u_{3}+\left(q-p_{1}\right) \cdot k \bar{u}_{2} \gamma^{[\alpha} u_{1} \bar{u}_{4} \gamma^{\beta]} u_{3}-\frac{q \cdot k}{4} \bar{u}_{2} \gamma^{[\alpha} \gamma^{\beta]} \gamma^{\mu} u_{1} \bar{u}_{4} \gamma_{\mu} u_{3}\right], \\
& n_{4}^{\prime\left(\frac{1}{2}, \frac{1}{2}\right)}=\frac{4 e^{3} F_{\alpha \beta}}{q \cdot k}\left[q^{[\alpha} p_{3}^{\beta]} \bar{u}_{4} \gamma^{\mu} u_{3} \bar{u}_{2} \gamma_{\mu} u_{1}+\left(q+p_{3}\right) \cdot k \bar{u}_{4} \gamma^{[\alpha} u_{3} \bar{u}_{2} \gamma^{\beta]} u_{1}-\frac{q \cdot k}{4} \bar{u}_{4} \gamma^{[\alpha} \gamma^{\beta]} \gamma^{\mu} u_{3} \bar{u}_{2} \gamma_{\mu} u_{1}\right] .
\end{aligned}
$$

Analogously, their charge conjugated pairs read

$$
\begin{aligned}
& \bar{n}_{1}^{\prime\left(\frac{1}{2}, \frac{1}{2}\right)}=\frac{4 e^{3}}{q \cdot k} F_{\alpha \beta}\left[q^{[\alpha} p_{1}^{\beta]} \bar{v}_{1} \gamma^{\mu} v_{2} \bar{v}_{3} \gamma_{\mu} v_{4}+\left(q-p_{1}\right) \cdot k \bar{v}_{1} \gamma^{[\alpha} v_{2} \bar{v}_{3} \gamma^{\beta]} v_{4}+\frac{q \cdot k}{4} \bar{v}_{1} \gamma^{\mu} \gamma^{[\alpha} \gamma^{\beta]} v_{2} \bar{v}_{3} \gamma_{\mu} v_{4}\right], \\
& \bar{n}_{4}^{\prime\left(\frac{1}{2}, \frac{1}{2}\right)}=\frac{4 e^{3}}{q \cdot k} F_{\alpha \beta}\left[q^{[\alpha} p_{3}^{\beta]} \bar{v}_{3} \gamma^{\mu} v_{4} \bar{v}_{1} \gamma_{\mu} v_{2}+\left(q+p_{3}\right) \cdot k \bar{v}_{3} \gamma^{[\alpha} v_{4} \bar{v}_{1} \gamma^{\beta]} v_{2}+\frac{q \cdot k}{4} \bar{v}_{3} \gamma^{\mu} \gamma^{[\alpha} \gamma^{\beta]} v_{4} \bar{v}_{1} \gamma_{\mu} v_{2}\right] .
\end{aligned}
$$

The gravitational amplitude $M_{5}^{\left(\frac{1}{2} \otimes \frac{1}{2}, \frac{1}{2} \otimes \frac{1}{2}\right)}$ can be computed from the double copy of the above numerators with their charge conjugated pairs, using the operation defined in (4.7). The result, which we provide in the Mathematica notebook in the supplementary material, is in complete agreement with the Feynman diagrammatic computation from the Lagrangian (4.21).

On the classical side, although the classical limit of these QCD numerators agrees with (4.55) (with appropiate conjugated numerators and up to dipole order), it is clear that the double copy $\left\langle M_{5}^{\left(\frac{1}{2} \otimes \frac{1}{2}, \frac{1}{2} \otimes \frac{1}{2}\right)}\right\rangle$ differs from $\left\langle M_{5}^{(0 \otimes 1,0 \otimes 1)}\right\rangle$. For instance, as the double copy for the former is symmetric in the numerators the axion field has no radiative amplitude, whereas for the latter it is unavoidably present.

We do not provide the explicit result for $\left\langle M_{5}^{\left(\frac{1}{2} \otimes \frac{1}{2}, \frac{1}{2} \otimes \frac{1}{2}\right)}\right\rangle$, but let us mention that it is naturally computed using the symmetric double copy product defined in [47], which preserves the multipole structure of the amplitude.

\section{Discussion}

Based on the analysis performed along refs. $[56,59,96,99,101]$ and in the current work we can draw an equivalence for lower spins between the following three statements:

1. The cancellation of $\frac{1}{m}$ divergences in the tree-level high-energy limit of single matter lines.

2. The "natural value" of the gyromagnetic ratio $g=2$.

3. The double copy construction for the single matter line $\left(A_{n}\right)$ amplitudes.

Let us remark that this equivalence not only seems to show up in QFT amplitudes but also in classical solutions [96]. One instance of this is the so-called $\sqrt{\mathrm{Kerr}}$ solution in electrodynamics which has been the focus of recent studies $[12,52]$. This EM solution can be double-copied into the Kerr metric via the Kerr-Schild ansatz [126], and also features 
$g=2$. Since these classical solutions contain the full tower of spin-multipoles, and so do higher spin particles in QFT, a natural question that arises is: How much of the above equivalence can be promoted to higher spins?

A hint of the answer may come from the 3-pt amplitudes first derived in [53] which are directly related to the aforementioned classical solutions [12, 33, 50-52, 104], at least at leading order in the coupling. In [47] we have emphasized their double copy structure, which fixes not only $g=2$ but also the full tower of multipoles in both gravity and QCD side. Here we have pointed out that these objects are in correspondence with higher spins massless amplitudes, thereby providing an underlying reason for double copy. Quite paradoxically, the latter are known to be inconsistent [95] whereas the former have an striking physical realization. To elucidate this contradiction we recall that massless higher spin amplitudes only fail at the level of the "4-particle" test [53, 95].

Indeed, the higher spin 4-point (Compton) $A_{4}$ amplitudes suffer from ambiguities in the form of contact terms and from $\frac{1}{m}$ divergences, although recent progress to understand these has been made in $[33,47,50,51]$. The importance of this object at higher spins was emphasized by one of us in [104] and proposed to control the subleading order associated to gravitational and EM classical potentials. These potentials emerge in the two-body problem $[33,48,51,107,125,127,128]$ (particularly outside the test body limit) and hence their understanding could have not only theoretical but practical implications. In fact, the relevance of the full tower of $A_{n}$ amplitudes lies in that they have been proposed to control the classical piece of conservative potentials at deeper orders in the coupling [32, 48, 49, 80, 129].

In [47] we demonstrated the latter fact is true also for radiation: at least at order $\sim \kappa^{3}$ and at spins $s \leq 2$ the non-conservative observables are controlled by $A_{4}$ and $A_{3}$ instead of the full $M_{5}$ amplitude. Here we have rederived this construction from a BCJ double-copy perspective and use it to make contact with the results of Goldberger et al. [22-24, 26, 27] for the full massless spectrum including dilatons, axions and gravitons. As we have mentioned it is remarkable how via QFT double copy we have found the precise couplings of these fields to matter, besides the aforementioned $g=2$ condition. One such extensions has naturally led us to $\mathcal{N}=4$ Supergravity seen as a classical theory of long-range forces. On the practical side it is important to evaluate the relevance of these additional fields, as well as string theory corrections, from the perspective of effective classical potentials arising from amplitudes, see e.g. [130, 131] for recent related results.

As a last point, let us mention that even though the cancellation of $\frac{1}{m}$ divergences at higher spins has been ruled out [61,99] it is still true that the choice of $g=2$ is preferred from both a causality perspective and the counting of degrees of freedom in certain cases $[81,96,99,101]$. It would be interesting to see if such cases are to exhibit a double copy structure: in fact the higher-spin 3-pt. amplitudes (2.35), when written in a local form using polarization tensors, also feature such $1 / m$ terms $[47,102,103]$. On the other hand, it is true that string theory provides a consistent tower of higher spin states exhibiting double copy [82]. In fact, on the open string side such states have $g=2$ [101] as implied by the double copy relations [51]. It remains to understand whereas a truncation of the full string spectrum to only certain higher spin states (for instance by isolating a single Regge trajectory [101]) is possible. 


\section{Acknowledgments}

We thank Freddy Cachazo, Henrik Johansson and Alex Ochirov for useful discussions. We thank Emilio Ojeda and Shan-Ming Ruan for their help with a Mathematica implementation. A.G. acknowledges support via Conicyt grant 21151647. Y.F.B. acknowledges the Natural Sciences and Engineering Research Council of Canada (NSERC) the financial support. Research at Perimeter Institute is supported by the Government of Canada through the Department of Innovation, Science and Economic Development Canada and by the Province of Ontario through the Ministry of Research, Innovation and Science.

\section{A Double copy in $d=4$}

In this appendix we outline the $\frac{1}{2} \otimes \frac{1}{2}$ construction in $d=4$. It is interesting to make connection with the spinor formalism for massive particles [53], recently implemented for obtaining a massive double copy in [79]. Let us briefly sketch how our operation will read in such variables. For this, observe that we can write

$$
\left.E_{\mu}^{a b} \sigma^{\mu}=\frac{\sqrt{2}}{m} \mid p^{(a}\right]\left\langle p^{b)}\left|\quad E_{\mu}^{a b} \tilde{\sigma}^{\mu}=\frac{\sqrt{2}}{m}\right| p^{(a}\right\rangle\left[p^{b)} \mid .\right.
$$

where $E_{\mu}^{a b}$ is a spin- 1 polarization vector, $\tilde{E}^{a b} \cdot P=0$, with the little group indices $\{a, b\}=$ $\{1,1\},\{2,2\},\{1,2\}$. Note its spinors satisfy the Dirac equation

$$
\left.\left.P\left|p^{a}\right\rangle=m \mid p^{a}\right], \quad \tilde{P} \mid p^{a}\right]=m\left|p^{a}\right\rangle,
$$

where $P=P_{\mu} \sigma^{\mu}$ and $\tilde{P}=P_{\mu} \tilde{\sigma}^{\mu}$. Then it is true that $\left[1^{a}, 1^{b}\right]=-m \epsilon^{a b}$, and $\left\langle 1^{a}, 1^{b}\right\rangle=m \epsilon^{a b}$ Now, in terms of the Dirac matrices note that

$$
\begin{aligned}
& \left(\not P+m \mathbb{I}_{4 \times 4}\right) \mathbb{E}^{a b}=\frac{\sqrt{2}}{m}\left(\begin{array}{cc}
m \mathbb{I}_{2 \times 2} & P \\
\tilde{P} & m \mathbb{I}_{2 \times 2}
\end{array}\right)\left(\begin{array}{cc}
0 & \left.\mid 1^{(a}\right]\left\langle 1^{b)}\right| \\
\left|1^{(a}\right\rangle\left[1^{b)} \mid\right. & 0
\end{array}\right),
\end{aligned}
$$

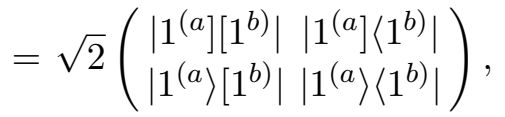

$$
\begin{aligned}
& =\sqrt{2}\left(\begin{array}{l}
\left.\mid 1^{(a}\right] \\
\left|1^{(a}\right\rangle
\end{array}\right)\left(\left[1^{b)} \mid\left\langle 1^{b)}\right|\right)\right. \\
& =\sqrt{2} u^{(a} \bar{v}^{b)} \text {, }
\end{aligned}
$$

where $u$ and $v$ are Dirac spinors satisfying $\not P u=m u, \not P v=-m v$, as follows from (A.2). Note that the spin-1 polarization can be recovered from (A.6) via

$$
E_{\mu}^{a b}=\frac{1}{\sqrt{2} m} \bar{v}^{(a} \gamma_{\mu} u^{b)}
$$

In this sense the spin-1 polarization vector is constructed out of spin- $\frac{1}{2}$ polarizations, analogously to the rules (2.31) for higher spins.

We see that in $d=4$ the choice of polarizations given by (A.1) turns the product (2.13) into

$$
X \otimes Y=\bar{v}_{2}^{\left(b_{2}\right.} X u_{1}^{\left(a_{1}\right.} \times \bar{v}_{1}^{\left.b_{1}\right)} \bar{Y} u_{2}^{\left.a_{2}\right)},
$$


which is simple multiplication together with symmetrization over the spin- $\frac{1}{2}$ states. Since this operation coincides with the one given in [79] we conclude that the amplitudes for a spin-1 field will agree in $d=4$.

For instance, for one matter line we will write

$$
A_{n}^{\mathrm{gr}}\left(E_{1}^{a_{1} b_{1}}, E_{2}^{a_{2} b_{2}}\right)=\sum_{\alpha, \beta} K_{\alpha \beta}\left(A_{n, \alpha}^{\mathrm{QCD}}\right)^{\left(a _ { 1 } \left(b_{2}\right.\right.}\left(A_{n, \beta}^{\mathrm{QCD}}\right) a_{2}^{\left.\left.a_{2}\right) b_{1}\right)} .
$$

which exhibits the symmetry properties of the indices explicitly. In particular it can be used to streamline the argument given in section 2 for axion pair-production. have

In an analogous way to (A.3), in the representation where $\gamma^{5}=\left(\begin{array}{cc}-\mathbb{I}_{2 \times 2} & 0 \\ 0 & \mathbb{I}_{2 \times 2}\end{array}\right)$, we

$$
\begin{aligned}
\left(\not P+m \mathbb{I}_{4 \times 4}\right) \gamma^{5} & =\left(\begin{array}{cc}
-m \mathbb{I}_{2 \times 2} & \mid 1]^{a}\left\langle\left. 1\right|^{b} \epsilon_{a b}\right. \\
|1\rangle^{a}\left[\left.1\right|^{b} \epsilon_{a b}\right. & m \mathbb{I}_{2 \times 2}
\end{array}\right) \\
& =u^{[a} \bar{v}^{b]} \epsilon_{a b} .
\end{aligned}
$$

By inserting the projector on the l.h.s. instead of (A.3) into our double copy, we find that antisymmetrizing little group indices from the Dirac spinors leads to a pseudoscalar. This antisymmetrization will necessarily require an odd number of axion fields in (A.9). Hence the axion can be sourced by matter if the Proca field decays to a pseudoscalar, which is again consistent with the Lagrangian of [79]. Further analysis in general dimensions is done in the next appendix.

Lagrangian comparison with [79]. The results of [79] consider the full spectrum of the $1 / 2 \otimes 1 / 2$ double copy restricted to four-dimensions. In contrast, our work shows that there exists a truncated spectrum in general dimensions. It is interesting to analyze the overlap by comparing the interactions in our Lagrangian (1.4) with a truncated version of the one in [79]. Note that in principle the matching at the level of amplitudes does not guarantee such an off-shell agreement due to diverse field redefinitions. However, in our case it is possible since 1) both actions are written on the Einstein frame for the graviton-dilaton couplings and 2) It can be shown that the axion and massive pseudoscalar fields decouple in the amplitudes of [79], hence the corresponding interaction terms can be ignored in their Lagrangian.

With the previous considerations the Lagrangian of [79] leads to the following explicit couplings of the dilaton to the Proca field at $\mathcal{O}\left(\kappa^{2}\right)$

$\mathcal{L}_{\mathrm{QCD}^{2}}=-\frac{2}{\kappa^{2}} R+\frac{\partial_{\mu} \bar{Z} \partial^{\mu} Z}{\left(1-\frac{\kappa^{2}}{4} \bar{Z} Z\right)}-\frac{1}{2} F_{\mu \nu}^{*} F^{\mu \nu}+m^{2} A_{\mu}^{*} A^{\mu}\left(1-\frac{\kappa}{2}(\bar{Z}+Z)+\frac{\kappa^{2}}{2} \bar{Z} Z+\mathcal{O}\left(\kappa^{3}\right)\right)$

The kinetic term for $Z$ can be cast into the standard form when we identify the dilaton component. Indeed, recall the field $Z$ was defined by

$$
Z=\frac{2 a+i\left(e^{-2 \phi}-1\right)}{2 a+i\left(e^{-2 \phi}+1\right)}
$$


Where the axion corresponds to the parity-odd piece, i.e. the field $a$. Setting $a \rightarrow 0$ implies $\bar{Z}=Z=-\tanh \phi$. Doing the further field redefinition $Z \rightarrow \frac{\kappa}{2} Z$, the Lagrangian (A.12) becomes

$$
\mathcal{L}_{\mathrm{QCD}^{2}}=-\frac{2}{\kappa^{2}} R+\frac{4}{\kappa^{2}}(\partial \phi)^{2}-\frac{1}{2} F_{\mu \nu}^{*} F^{\mu \nu}+m^{2} A_{\mu}^{*} A^{\mu}\left(1+2 \tanh \phi+2(\tanh \phi)^{2}+\mathcal{O}\left(\kappa^{3}\right)\right) .
$$

Finally, we do the field re-definition $\phi \rightarrow \frac{\kappa}{2} \phi$, expanding up to second order in $\phi$, which is the order of the validity of the Lagrangian (A.12); in addition, we turn $A^{\mu}$ into a real field using the argument made above (3.5), to arrive at

$$
\mathcal{L}_{\mathrm{QCD}^{2}}=-\frac{2}{\kappa^{2}} R+(\partial \phi)^{2}-\frac{1}{4} F_{\mu \nu} F^{\mu \nu}+\frac{m^{2}}{2} A_{\mu} A^{\mu}\left(1+\kappa \phi+\frac{\kappa^{2}}{2} \phi^{2}+\mathcal{O}\left(\kappa^{3}\right)\right),
$$

which precisely agrees with the Lagrangian (1.4) for $d=4$ if we truncate at $\mathcal{O}\left(\kappa^{2}\right)$.

\section{B Tree-level unitarity at $n=4$}

In this appendix we compute the residues of the gravitational amplitude $A_{4}^{\frac{1}{2} \otimes \frac{1}{2}}$. The aim of this is twofold. On the one hand this checks explicitly that the operation (2.11) defines a QFT amplitude for $n=4$ and outlines the argument for general $n$. On the other hand, we want to find the matter fields that propagate in a given factorization channel. For two dilaton emissions we find only the propagation of the Proca field, which is consistent with our Lagrangian (3.30). For two axion emissions we find the propagation of tensor structures of rank four and five. The former can be interpreted as a pseudoscalar in $d=4$. In general dimension, the propagation of these structures makes it more involved to write the Lagrangian of the full $\frac{1}{2} \otimes \frac{1}{2}$ theory including axions.

Consider then the Compton amplitude from the $\frac{1}{2} \otimes \frac{1}{2}$ theory (2.11)

$$
A_{4}^{\frac{1}{2} \otimes \frac{1}{2}}\left(W_{1} H_{3}^{\mu_{3} \nu_{3}} H_{4}^{\mu_{4} \nu_{4}} W_{2}^{*}\right)=\frac{K_{1324,1324}}{2^{\lfloor d / 2\rfloor-1}} \operatorname{tr}\left[A_{4,1324}^{\mathrm{QCD}, \mu_{3} \mu_{4}} \notin_{1}(p / 1+m) \bar{A}_{4,1324}^{\mathrm{QCD}, \nu_{3} \nu_{4}} \notin_{2}(p / 2+m)\right],
$$

where the 4 pt. QCD partial amplitudes are given in (2.18), and the massive KLT kernel at four points was given in (2.21).

We have claimed that (B.1) defines a tree-level amplitude. First, from the standard argument it is clear that the r.h.s. is local. Let us then argue that unitarity of the gravitational amplitude follows from the unitarity of the QCD amplitudes. Consider for instance the factorization channel $2 p_{1} \cdot k_{3} \rightarrow 0$. We know that in such case the QCD amplitude factorizes as

$$
A_{4,1324}^{\mathrm{QCD}, \mu_{3} \mu_{4}} \rightarrow \frac{1}{2 p_{1} \cdot k_{3}} A_{3, \mathrm{R}}^{\mathrm{QCD}, \mu_{3}}\left(\not p_{13}-m\right) A_{3, \mathrm{~L}}^{\mathrm{QCD}, \mu_{4}}+\cdots,
$$

Analogously, the charge conjugated amplitude factorizes as

$$
\bar{A}_{4,1324}^{\mathrm{QCD}, \nu_{3} \nu_{4}} \rightarrow \frac{1}{2 p_{1} \cdot k_{3}} \bar{A}_{3, \mathrm{~L}}^{\mathrm{QCD}, \nu_{4}}\left(\not p_{13}+m\right) \bar{A}_{3, \mathrm{R}}^{\mathrm{QCD}, \nu_{3}}+\cdots
$$


This implies that (B.1) behaves as

$$
\begin{array}{r}
A_{4}^{\frac{1}{2} \otimes \frac{1}{2}}\left(W_{1} H_{3}^{\mu_{3} \nu_{3}} H_{4}^{\mu_{4} \nu_{4}} W_{2}^{*}\right) \rightarrow-\frac{1}{2 p_{1} \cdot k_{3} 2^{\lfloor d / 2\rfloor-1}} \operatorname{tr}\left[A_{3, \mathrm{~L}}^{\mathrm{QCD}, \mu_{4}} \notin_{1}\left(p p_{1}+m\right) \bar{A}_{3, \mathrm{~L}}^{\mathrm{QCD}, \nu_{4}}\left(\not p_{13}+m\right)\right. \\
\left.\left.\times \bar{A}_{3, \mathrm{R}}^{\mathrm{QCD}, \nu_{3}} \notin_{2}\left(\not p_{2}+m\right) A_{3, \mathrm{R}}^{\mathrm{QCD}, \mu_{3}}\left(\not p_{13}-m\right)\right]+\cdots, \quad \text { (B. } 4\right)
\end{array}
$$

We can examine the inner spectrum in the factorization channel by using the Fierz relations for the product of two matrices $M$ and $N$ [132],

$$
\operatorname{tr}[M \times N]=\frac{1}{2^{\lfloor d / 2\rfloor}} \sum_{J}^{[d]} \frac{(-1)^{|J|}}{|J| !} \operatorname{tr}\left[M \Gamma_{J}\right] \operatorname{tr}\left[N \Gamma^{J}\right], \quad[d]= \begin{cases}d & \text { for even } d \\ \frac{d-1}{2} & \text { for odd } d\end{cases}
$$

where $\left\{\Gamma^{J}=\mathbb{I}, \gamma^{\alpha}, \gamma^{\alpha_{1} \alpha_{2}}, \cdots, \gamma^{\alpha_{1} \cdots \alpha_{d}}\right\}$ is the Clifford algebra basis, with $\alpha_{1}<\alpha_{2}<\cdots<$ $\alpha_{r}$. The gravitational amplitude (B.4) then takes the form

$$
\begin{gathered}
-\frac{1}{4 p_{1} \cdot k_{3} 2^{2\lfloor d / 2\rfloor-2}} \sum_{J}^{[d]} \frac{(-1)^{|J|}}{|J| !} \operatorname{tr}\left[A_{3, \mathrm{~L}}^{\mathrm{QCD}, \mu_{4}} \notin_{1}(\not p 1+m) \bar{A}_{3, \mathrm{~L}}^{\mathrm{QCD}, \nu_{4}}\left(\not p_{13}+m\right) \Gamma_{J}\right] \\
\times \operatorname{tr}\left[\bar{A}_{3 \mathrm{R}}^{\mathrm{QCD}, \nu_{3}} \notin_{2}\left(\not p_{2}+m\right) A_{3, \mathrm{R}}^{\mathrm{QCD}, \mu_{3}}\left(\not p_{13}-m\right) \Gamma^{J}\right]+\cdots
\end{gathered}
$$

Now it is clear that each trace corresponds to the double copy for the 3pt amplitudes, therefore we have

$$
A_{4}^{\frac{1}{2} \otimes \frac{1}{2}}\left(W_{1} H_{3}^{\mu_{3} \nu_{3}} H_{4}^{\mu_{4} \nu_{4}} W_{2}^{*}\right) \rightarrow-\frac{1}{4 p_{1} \cdot k_{3}} \sum_{J}^{[d]} \frac{(-1)^{|J|}}{|J| !} A_{3, \mathrm{~L}}^{\frac{1}{2} \otimes \frac{1}{2}}\left(W_{1} H_{3}^{\mu_{3} \nu_{3}} \Phi_{J}\right) \times A_{3, \mathrm{R}}^{\frac{1}{2} \otimes \frac{1}{2}}\left(\Phi^{J} H_{4}^{\mu_{4} \nu_{4}} W_{2}^{*}\right) .
$$

Hence, we have shown that the gravitational 4-pt. amplitude factorizes into the product of two 3-pt. amplitudes. Moreover, $\Phi_{J}$ indicates all possible Lorentz structure propagating in the given factorization channel. We can expand the sum to see the explicit form of some of these structures propagating in this channel. To do so, first notice that since $\left(\not p_{13}+m\right) \mathbb{I}=\frac{p_{13, \alpha}}{m}\left(\not p_{13}+m\right) \gamma^{\alpha}$, we can identify the contribution from the terms $|J|=0$ and $|J|=1$ with the transverse and longitudinal modes of the spin-1 field. With this consideration (B.7) takes the form

$$
\begin{aligned}
& -\frac{1}{p_{1} \cdot k_{3} 2^{2\lfloor d / 2\rfloor}}\left\{\operatorname{tr}\left[A_{3, \mathrm{~L}}^{\mathrm{QCD}, \mu_{4}} \notin_{1}\left(\not p_{1}+m\right) \bar{A}_{3, \mathrm{~L}}^{\mathrm{QCD}, \nu_{4}}\left(\not p_{13}+m\right) \gamma^{\alpha}\right] D_{W, \alpha \beta}\right. \\
& \quad \times \operatorname{tr}\left[\bar{A}_{3, \mathrm{R}}^{\mathrm{QCD}, \nu_{3}} \notin_{2}\left(\not p_{2}+m\right) A_{3, \mathrm{R}}^{\mathrm{QCD}, \mu_{3}}\left(\not p_{13}-m\right) \gamma^{\beta}\right] \\
& \quad+\frac{1}{2} \operatorname{tr}\left[A_{3, \mathrm{~L}}^{\mathrm{QCD}, \mu_{4}} \notin_{1}\left(\not p_{1}+m\right) \bar{A}_{3, \mathrm{~L}}^{\mathrm{QCD}, \nu_{4}}\left(\not p_{13}+m\right) \gamma^{\mu \nu}\right] \\
& \left.\quad \times \eta_{[\mu \alpha} \eta_{\nu] \beta} \operatorname{tr}\left[\bar{A}^{-} \mathrm{R}_{3}^{\mathrm{QCD}, \nu_{3}} \notin_{2}\left(\not p_{2}+m\right) A_{3, \mathrm{R}}^{\mathrm{QCD}, \mu_{3}}\left(\not p_{13}-m\right) \gamma^{\alpha \beta}\right]+\cdots\right\},
\end{aligned}
$$

where

$$
D_{W, \alpha \beta}=\eta_{\alpha \beta}-\frac{p_{13, \alpha} p_{13, \beta}}{m^{2}},
$$

and the $\cdots$ indicate the terms with higher value of $|J|$. 
A similar analysis can be made at higher multiplicity starting from (2.11). The additional complication is that we have to deal with the factorization of the KLT kernel $K_{\alpha \beta}$, which is however standard. Once the dust settles we obtain

$$
\begin{gathered}
\left.-\frac{1}{2\left(p_{I}^{2}-\right.} m^{2}\right) 2^{2\lfloor d / 2\rfloor-2} \sum_{J}^{[d]} \frac{(-1)^{|J|}}{|J| !} K_{\alpha_{L} \beta_{L}} \operatorname{tr}\left[A_{n_{L}, \alpha_{L}}^{\mathrm{QCD}} \ddagger_{1}(\not p / 1+m) \bar{A}_{n_{L}, \beta_{L}}^{\mathrm{QCD}}\left(\not p_{I}+m\right) \Gamma_{J}\right] \\
\times K_{\alpha_{R} \beta_{R}} \operatorname{tr}\left[\bar{A}_{n_{R}, \beta_{R}}^{\mathrm{QCD}} \ddagger_{2}\left(\not p_{2}+m\right) A_{n_{R}, \beta_{R}}^{\mathrm{QCD}}\left(\not p_{I}-m\right) \Gamma^{J}\right]+\cdots,
\end{gathered}
$$

as $p_{I}^{2} \rightarrow m^{2}$, for $p_{I}$ any internal massive momenta. This means that unitarity of $A_{n}^{\frac{1}{2} \otimes \frac{1}{2}}$ should follow from that of $A_{n}^{\mathrm{QCD}}$ provided we correcltly account for the tensor structures $\Gamma^{J}$ as particles propagating in this channel.

Let us leave the analysis for general multiplicity for future work, and here instead focus in the internal spectrum at $n=4$. Next we consider two such cases and determine the fields propagating in this channel. The first is the gravitational amplitude for a massive line emitting two dilatons, whereas the second one corresponds to the amplitude for the emission of two axions.

Dilaton emission. For this explicit example the sum truncates at $|J|=3$. Moreover, it can be checked that the terms $|J|=2$ and $|J|=3$ add up exactly to the contributions given by the $|J|=0$ and $|J|=1$ terms, namely, they account for a propagating spin-1 field. With this in mind, (B.7) gives

$$
A_{4}^{\frac{1}{2} \otimes \frac{1}{2}}\left(W_{1} \phi_{3} \phi_{4} W_{2}^{*}\right) \rightarrow \frac{\kappa^{2}}{32 p_{1} \cdot k_{3}(2-d)}\left[(d-4) p_{1}^{\alpha} p_{3} \cdot \varepsilon_{1}+2 m^{2} \varepsilon_{1}^{\alpha}\right] D_{W, \alpha \beta}\left[(d-4) p_{2}^{\beta} p_{4} \cdot \varepsilon_{2}+2 m^{2} \varepsilon_{2}^{\beta}\right] .
$$

It can be also checked that the same residue is computed starting from (2.22).

Axion emission. Let us move on to the slightly more complicated example corresponding to the emission of two axions by a massive line. As we mentioned, the matter spectrum of the $\frac{1}{2} \otimes \frac{1}{2}$ double copy can be truncated to massive vector fields once we consider the emission of gravitons or dilatons, but not axions. On the other hand, via double copy we showed that the matter line can only produce axions in pairs. An example of this is the four point amplitude for two axions:

$$
A_{4}^{\frac{1}{2} \otimes \frac{1}{2}}\left(W_{1} B_{3} B_{4} W_{2}^{*}\right)=\frac{1}{2^{\lfloor d / 2\rfloor-1}} K_{1324,1324}\left(A_{4,1324\left[\mu_{4}\right.}^{\mathrm{QCD},\left[\mu_{3}\right.} \bar{A}_{\left.4,1324 \nu_{4}\right]}^{\left.\mathrm{QCD}, \nu_{3}\right]}\right) \epsilon_{B_{3}, \mu_{3} \nu_{3}} \epsilon_{B_{4}}^{\mu_{4} \nu_{4}} .
$$

Studying tree-level unitarity in this object leads to consider additional matter fields. For instance, consider the channel $2 p_{1} \cdot k_{3} \rightarrow 0$ given by (B.7). For two axion emissions, the sum truncates at $|J|=5$. The sum of the contributions for $|J|=0$ and $|J|=1$ cancels out, therefore no Proca field will propagate in this channel, as expected since $A_{3}^{\frac{1}{2} \otimes \frac{1}{2}}\left(W_{1} B W_{2}^{*}\right)=$ 0 . We can check that the sum of the contributions for $|J|=2$ and $|J|=3$ equals the sum of the contributions for $|J|=4$ and $|J|=5$. Therefore, in this factorization channel there is the propagation of particles associated to the structures $\left\{\gamma^{\mu_{1}, \mu_{2}}, \gamma^{\mu_{1} \mu_{2} \mu_{3}}\right\}$ or equivalently $\left\{\gamma^{\mu_{1} \mu_{2} \mu_{3} \mu_{4}}, \gamma^{\mu_{1} \mu_{2} \mu_{3} \mu_{4} \mu_{5}}\right\}$. The propagation of these structures is what makes more involved 
to write down a Lagrangian including the additional fields in general dimension. We leave this task for future work. In $d=4$ however there is a simplification since the form $\gamma^{\mu_{1} \mu_{2} \mu_{3} \mu_{4}}$ can be dualized to a pseudoscalar, whereas the form $\gamma^{\mu_{1} \mu_{2} \mu_{3} \mu_{4} \mu_{5}}$ vanishes. The propagation of this pseudoscalar (as obtained in [79]) was pointed out in the previous appendix, as obtained from antisymmetrization of spinors in $d=4$.

\section{C $\mathcal{N}=4$ SUGRA in the form of Nicolai and Townsend}

In this appendix we review the original construction of [133]. There the axion pseudoscalar was dualized to a two-form at the level of the Lagrangian, i.e. off-shell. The starting point is the following bosonic action

$\mathcal{L}^{\mathcal{N}=4}=\sqrt{g}\left[R-2(\partial \phi)^{2}-2 e^{4 \phi} X_{\mu} X^{\mu}-e^{-2 \phi} F_{\mu \nu}^{I} F_{I}^{\mu \nu}\right]+2 \epsilon^{\mu \nu \rho \sigma} X_{\mu} A_{I \nu} F_{\rho \sigma}^{I}+\epsilon^{\mu \nu \rho \sigma} B_{\mu \nu} \partial_{\rho} X_{\sigma}$.

Here $B_{\mu \nu}$ acts as a Lagrange multiplier imposing the condition

$$
0=\frac{\delta \mathcal{L}}{\delta B_{\mu \nu}}=\epsilon^{\mu \nu \rho \sigma} \partial_{\rho} X_{\sigma}
$$

We can solve such constraint locally by $X_{\mu}=\partial_{\mu} \chi$ (and globally provided certain topological conditions) and plug it back on the action. Then, $\chi$ can be seen as a dynamical pseudoscalar carrying the degrees of freedom of the axion in four dimensions. The resulting Lagrangian then reads

$$
\left.\mathcal{L}^{\mathcal{N}=4}\right|_{X_{\mu}=\partial_{\mu} \chi}=\sqrt{g}\left[R-2(\partial \phi)^{2}-2 e^{4 \phi}(\partial \chi)^{2}-e^{-2 \phi} F_{\mu \nu}^{I} F_{I}^{\mu \nu}-2 \chi F_{\mu \nu}^{I} \star F_{I}^{\mu \nu}\right],
$$

where $\star F_{I}^{\mu \nu}=\frac{1}{2 \sqrt{g}} \epsilon^{\mu \nu \rho \sigma} F_{\rho \sigma}^{I}$ is the Hodge dual. This is the standard form of the $\mathcal{N}=4$ Supergravity action given in the Einstein frame, introduced in $[74,75]$. A small paradox arises in that from the analysis of the main text we expect to find contact matter interactions between flavours. However, in this case we have six flavours of photons which only interact through massless exchanges. The resolution of the paradox is that this is not a well posed statement, as we will see when integrating out the scalar $\chi$, or more precisely, replacing it by the two form $B_{\mu \nu}$. In order to do so we go back to C.1 and solve the field equations of $X_{\mu}$ instead:

$$
X^{\mu}=\frac{e^{-4 \phi}}{2 \sqrt{g}} \epsilon^{\mu \nu \rho \sigma}\left[A_{\nu}^{I} F_{I \rho \sigma}+\frac{1}{6} H_{\rho \sigma \nu}\right] \Rightarrow X_{\mu}=-\frac{e^{-4 \phi}}{2} \sqrt{g} \epsilon_{\mu \nu \rho \sigma}\left[A^{I \nu} F_{I}^{\rho \sigma}+\frac{1}{6} H^{\rho \sigma \nu}\right],
$$

where $H=d B$. Inserting this back in (C.1) gives, after some algebra,

$$
\sqrt{g}\left[R-2(\partial \phi)^{2}+3 e^{-4 \phi}\left(A_{I}^{\nu} F^{I \rho \sigma}+\frac{1}{6} H^{\nu \rho \sigma}\right)\left(A_{J \nu} F_{\rho \sigma}^{J}+\frac{1}{6} H_{\nu \rho \sigma}\right)-e^{-2 \phi} F_{\mu \nu}^{I} F_{I}^{\mu \nu}\right],
$$

which leads to the interaction tern $\sim A^{2} F^{2}$. Finally, note that the original Lagrangian (C.3) is invariant under $\delta A_{\mu}^{I}=-\partial_{\mu} \xi^{I}$ whereas the dualized one (C.5) seems not to be. This is 
reconciled by imposing that $X^{\mu}$ in (C.4) does not change under the $\mathrm{U}(1)^{6}$ gauge transformations, which in turn can be achieved via

$$
\delta B_{\mu \nu}=2 \xi_{I} F_{\mu \nu}^{I} \Rightarrow \delta H_{\mu \nu \rho}=6 \partial_{[\mu} \xi_{I} F_{\nu \rho]}^{I} .
$$

However, after restoring the coupling $\kappa$ as in the main text we find that $\delta B_{\mu \nu}=\mathcal{O}(\kappa)$ and hence the gauge symmetry does not shift the asymptotic axion states.

\section{Testing amplitudes from CHY-like formulas}

To construct the KLT product while taking different traces (Dirac traces or Lorentz traces for dilatons) can be a cumbersome operation. Hence in this section we will provide CHYlike formulas that automatically implement double copy and at the same time avoid the computation of QCD Feynman diagrams. For the case of the $\frac{1}{2} \otimes \frac{1}{2}$ theory we will more precisely use the connected prescription (as in [134] for the massless case) which was recently introduced for massive particles in [70, 71] and unified in [72].

\section{D.1 The $0 \otimes 1$ theory}

Based on the considerations of the main text it is direct to identify the massless version of the $0 \otimes 1$ theory with the extended "Einstein-Maxwell" theory considered in the context of CHY in [68]. Thus our conjecture is to assign the $d$-dimensional Lagrangian (3.42) to such construction, even for the massive case. As a warm-up for the $\frac{1}{2} \otimes \frac{1}{2}$ case we give an overview on this construction.

In the CHY formulation [135] the amplitude is obtained by solving the scattering equations $E_{i}=0, i=1, \ldots, n$, where

$$
E_{i}:=\sum_{j \neq i} \frac{2 P_{i} \cdot P_{j}}{\sigma_{i j}}, \quad \sigma_{i j}=\sigma_{i}-\sigma_{j}
$$

These equations feature an $\mathrm{SL}(2, \mathbb{C})$ redundancy due to the fact that $\sum_{j \neq i} P_{i} \cdot P_{j}=0$ which requires the momenta to be massless $P_{i}^{2}=0$, and in fact only $(n-3)$ of the $E_{i}$ 's are independent. The gravitational and YM amplitudes are given by

$$
\begin{aligned}
A_{n}^{\mathrm{gr}} & =\int \frac{\prod_{i=1}^{n} d \sigma_{i}}{\operatorname{Vol}(\mathrm{SL}(2, \mathbb{C}))} \prod_{i=1}^{n} \delta\left(E_{i}\right) \mathrm{Pf}^{\prime} \Psi_{n} \mathrm{Pf}^{\prime} \tilde{\Psi}_{n}, \\
A_{n}^{\mathrm{YM}}(\alpha) & =\int \frac{\prod_{i=1}^{n} d \sigma_{i}}{\operatorname{Vol}(\mathrm{SL}(2, \mathbb{C}))} \prod_{i=1}^{n} \delta\left(E_{i}\right) \operatorname{Pf}^{\prime} \Psi_{n} \mathrm{PT}(\alpha)
\end{aligned}
$$

where the different ingredients are detailed in $[66,67]$. Here we just need to recall that the delta functions $\delta\left(E_{i}\right)$ in fact localize the integration to $(n-3)$ ! solutions, hence making it effectively a sum over Jacobians weighted by the integrands. For $A_{n}^{\mathrm{YM}}(\alpha)$ the color ordering is encoded in the integrand

$$
\operatorname{PT}(\alpha):=\frac{1}{\left(\sigma_{\alpha_{1}}-\sigma_{\alpha_{2}}\right) \cdots\left(\sigma_{\alpha_{n}}-\sigma_{\alpha_{1}}\right)},
$$


whereas the polarization dependence is encoded in the reduced Pfaffian of the matrix $\Psi$. The double copy construction (2.11) is already implemented in (D.2): it corresponds to the replacement of one PT color actor by a second copy of the polarization factor $\mathrm{Pf}^{\prime} \tilde{\Psi}_{n}$. As observed in [69] we can directly compactify the polarization vectors in $\operatorname{Pf}^{\prime} \tilde{\Psi}_{n}$ as in (3.1) obtain the special YMS theory and the Einstein-Maxwell theory.

It was observed in [86] that the massive compactification can be implemented in the $\mathrm{CHY}$ formalism to include up to three massive interacting species. ${ }^{18}$ In our case we are solely interested in different species interacting only through massless exchanges. The corresponding massive scattering equations can be computed by setting the momenta as in (3.7).

As a preparation for next section let us give a simple example on how the scattering equations can be naturally adapted for massive particles. For two massive-lines of different species $a, b$ and no external massless fields, the only independent equation in (D.1) reads

$$
E_{1}=q^{2}+\frac{s-m_{a}^{2}-m_{b}^{2}}{1-\sigma}=0 \Longrightarrow \sigma=1+\frac{s-m_{a}^{2}-m_{b}^{2}}{q^{2}}
$$

where $q=p_{1}+p_{2}$ and $s=\left(p_{1}+p_{3}\right)^{2}$ and we have fixed $\left(\sigma_{1}, \ldots, \sigma_{4}\right)=(1,0, \sigma, a)$, with $a \rightarrow \infty$. It is straightforward to compute the four-massive amplitude $M_{4}$, we write

$$
\begin{aligned}
M_{4}^{(0,1)} & =\int \frac{\prod_{i=1}^{4} d \sigma_{i}}{\operatorname{Vol}(\operatorname{sl}(2, \mathbb{C}))} \prod_{i=1}^{4} \delta\left(E_{i}\right) \operatorname{Pf}^{\prime} \Psi_{4} \operatorname{Pf}^{\prime} \tilde{\Psi}_{4}^{C}, \\
& =\frac{\sigma(\sigma-1)}{q^{2}} \lim _{a \rightarrow \infty}\left(\operatorname{Pf}^{\prime} \Psi_{4} a^{2}\right)\left(\mathrm{Pf}^{\prime} \tilde{\Psi}_{4}^{C} a^{2}\right),
\end{aligned}
$$

where the factor $\lim _{a \rightarrow \infty}\left(\mathrm{Pf}^{\prime} \Psi_{4} a^{2}\right)$ is standard [67], whereas the second copy of this factor simplifies (under (3.1)) to

$$
\mathrm{Pf}^{\prime} \tilde{\Psi}_{4}^{C}=\operatorname{Pf}\left(\begin{array}{cccccc}
0 & \frac{q^{2}}{\sigma-a} & 0 & 0 & 0 & 0 \\
\frac{-q^{2}}{\sigma-a} & 0 & 0 & 0 & 0 & 0 \\
0 & 0 & 0 & 1 & 0 & 0 \\
0 & 0 & -1 & 0 & 0 & 0 \\
0 & 0 & 0 & 0 & 0 & \frac{1}{\sigma-a} \\
0 & 0 & 0 & 0 & \frac{-1}{\sigma-a} & 0
\end{array}\right)=\frac{q^{2}}{(\sigma-a)^{2}} \rightarrow \frac{q^{2}}{a^{2}}
$$

Hence we get to the compact form

$$
M_{4}^{(0,1)}=\sigma(\sigma-1) \lim _{a \rightarrow \infty}\left(\operatorname{Pf}^{\prime} \Psi_{4} a^{2}\right)=\frac{\left(s-m_{a}^{2}-m_{b}^{2}\right)\left(q^{2}+s-m_{a}^{2}-m_{b}^{2}\right)}{q^{2}} \times \lim _{a \rightarrow \infty}\left(\operatorname{Pf}^{\prime} \Psi_{4} a^{2}\right) .
$$

Of course, we have done nothing but to compactify a 4-graviton amplitude to obtain (D.6). However, at higher points the fact that we do not need to implement the KLT kernel explicitly turns out to be very efficient for testing our Lagrangian numerically. By including an external (fat) graviton, we can compute the 5-pt. amplitude $M_{5}$ in the compactified version of $\mathcal{N}=4$ SUGRA, whose classical limit matches with the amplitude considered in section 4.3.6.

\footnotetext{
${ }^{18}$ For a recent generalization of this procedure see [136].
} 


\section{D.2 The $\frac{1}{2} \otimes \frac{1}{2}$ theory}

Recently a rational map formalism has been introduced for 6D $(1,1)$ SYM Theory [70-72]. This can be understood as an extension of CHY that naturally produces superamplitudes in six dimensions [137], analogous to the Witten-RSV formalism in four dimensions [138-140]. Under dimensional reduction this theory generates the massive amplitudes of $d=4 \mathrm{SYM}$ in the Coulomb branch [141], which color-kinematics duality was first studied in [45]. On the other hand, the double copy is naturally incorporated into the rational map formula [70], in an analogous way to the original CHY construction [66].

As for one matter line the Coulomb branch amplitudes coincide with QCD, we can easily construct the $\frac{1}{2} \otimes \frac{1}{2}$ amplitudes in this framework. Presumably, we can also construct the amplitudes with two massive lines by applying the projections of [134, 142], although for this case we employ the BCJ prescription as in section 4 . On the other hand, the downside of the Witten-RSV picture is that we will have to restrict these checks to $d=4$ dimensions. In fact the amplitudes are naturally produced in the massive spinor-helicity formalism of [53], which we outlined already in appendix A.

For a review of the formalism see [72], which has also explained the equivalence of the two original formulations in [70, 71]. The massive SYM amplitudes can be written explicitly as the localized integral

$$
\begin{aligned}
\mathcal{A}_{n}^{\mathrm{SYM}}(\alpha) \delta^{4}\left(\sum_{i} p_{i}\right) \delta\left(\sum m_{i}\right) \delta\left(\sum \tilde{m}_{i}\right)= & \int d \mu \prod_{i=1}^{n} \delta^{2}\left(\sum_{j \neq i} \frac{\left\langle u_{j} u_{i}\right\rangle}{\sigma_{j i}} \lambda_{j}^{1}-\lambda_{i}^{1} v_{i}-\lambda_{i}^{1}\right) \\
& \times \delta^{2}\left(\sum_{j \neq i} \frac{\left\langle u_{j} u_{i}\right\rangle}{\sigma_{j i}} \tilde{\lambda}_{j}^{1}-\lambda_{i}^{1} v_{i}-\lambda_{i}^{1}\right) \operatorname{det}^{\prime}(H) \operatorname{PT}(\alpha) J_{F}
\end{aligned}
$$

where we have written the measure $d \mu=\frac{\prod_{i} d \sigma_{i} d v_{i} d^{2} u_{i}^{a}}{\operatorname{vol}\left(\operatorname{SL}(2, \mathbb{C})_{\sigma} \times \operatorname{SL}(2, \mathbb{C})_{u}\right)}$, and $\tilde{p}_{i}=\lambda_{i}^{a} \tilde{\lambda}_{i}^{b} \epsilon_{a b}$, omitting spinor indices ( $a, b$ are little-group indices). These satisfy $\left\langle\lambda_{i}^{1} \lambda_{i}^{2}\right\rangle=m_{i}$ and $\left[\tilde{\lambda}_{i}^{1} \tilde{\lambda}_{i}^{2}\right]=\tilde{m}_{i}$, so we can obtain massless particles (gluons) by taking $\lambda_{i}^{2}=\tilde{\lambda}_{i}^{1}=0$. The equations localizing the integration are named Polarized Scattering Equations [71, 143] and imply the (massive) scattering equations of the previous subsection. In the following we will take the volume form to be

$$
\operatorname{vol}\left(\operatorname{SL}(2, \mathbb{C})_{\sigma} \times \operatorname{SL}(2, \mathbb{C})_{u}\right)=d \sigma_{1} d \sigma_{2} d \sigma_{3} d^{2} u_{1}^{a} d u_{3}^{1} \times \sigma_{12} \sigma_{23} \sigma_{31} u_{1}^{1}\left\langle u_{1} u_{3}\right\rangle
$$

We will also need to define the matrix

$$
H_{i j}= \begin{cases}\frac{\left\langle\lambda_{i}^{1} \lambda_{j}^{1}\right\rangle-\left[\tilde{\lambda}_{i} \tilde{\lambda}_{j}\right]}{\sigma_{i j}} & i \neq j, \\ -\frac{1}{u_{i}^{2}} \sum_{k \neq i} \frac{u_{k}^{2}\left\langle\lambda_{i}^{1} \lambda_{k}^{1}\right\rangle-u_{k}^{2}\left[\tilde{\lambda}_{i} \tilde{\lambda}_{k}\right]}{\sigma_{i k}} & i=j,\end{cases}
$$

and $\operatorname{det}^{\prime}(H)=\frac{\operatorname{det}\left(H_{12,12}\right)}{\left\langle u_{1} u_{2}\right\rangle^{2}}$ where $H_{12,12}$ corresponds to $H$ with the first and second rows and columns deleted. The remaining object in the integrand is $J_{F}$, which depends on the external fields we are considering. For one matter line of massive fermions emitting gluons 
it is obtained via the Grassman integration

$$
\begin{aligned}
J_{F}^{s=\frac{1}{2}}=J_{F}\left(\psi^{a_{1}} g_{2}^{ \pm} \cdots g_{n-1}^{ \pm} \bar{\psi}^{b_{1}}\right)= & \int \prod_{i} d^{4} \eta_{i}^{a I} \times\left(\eta_{1}^{1,2} \eta_{1}^{2,2} \eta_{1}^{a_{1}, 1} \eta_{2}^{b_{1}, 1}\right) \prod_{r \in+} \eta_{r}^{2,1} \eta_{r}^{2,2} \prod_{s \in-} \eta_{s}^{1,1} \eta_{s}^{1,2} \\
& \times \prod_{i=1}^{n} \delta^{2}\left(\sum_{j \neq i} \frac{\left\langle u_{j} u_{i}\right\rangle}{\sigma_{j i}} \eta_{j}^{1, I}-\eta_{i}^{1, I} v_{i}-\eta_{i}^{1, I}\right)
\end{aligned}
$$

where $r(s)$ range over the positive (negative) helicity gluons. Implementation of this Jacobian is relatively direct using the Mathematica package MatrixEDC. ${ }^{19}$ The advantage is that we can now implement double copy of this object directly, instead of writing the KLT expansion and projecting into the states. It is obtained by the replacement of the integrand

$$
\operatorname{det}^{\prime}(H) \operatorname{PT}(\alpha) J_{F}^{s=\frac{1}{2}} \rightarrow \operatorname{det}^{\prime}(H)^{2} J_{F}^{s=1},
$$

where

$$
\begin{aligned}
J_{F}^{s=1}= & J_{F}\left(W^{a_{1} a_{2}} H_{2} \cdots H_{n-1} \bar{W}^{b_{1} b_{2}}\right)=\int \prod_{i} d^{4} \eta_{i}^{a I} d^{4} \tilde{\eta}_{i}^{a I}\left(\eta_{1}^{1,2} \eta_{1}^{2,2} \eta_{1}^{a_{1}, 1} \eta_{2}^{b_{1}, 1}\right)\left(\tilde{\eta}_{1}^{1,2} \tilde{\eta}_{1}^{2,2} \tilde{\eta}_{1}^{a_{1}, 1} \tilde{\eta}_{2}^{b_{1}, 1}\right) \\
& \times \prod_{t}\left[\eta_{j}^{2,1} \eta_{j}^{2,2} \tilde{\eta}_{j}^{1,1} \tilde{\eta}_{j}^{1,2}+\tilde{\eta}_{j}^{2,1} \tilde{\eta}_{j}^{2,2} \eta_{j}^{1,1} \eta_{j}^{1,2}\right] \prod_{r \in+} \eta_{r}^{2,1} \eta_{r}^{2,2} \tilde{\eta}_{r}^{2,1} \tilde{\eta}_{r}^{2,2} \prod_{s \in-} \eta_{s}^{1,1} \eta_{s}^{1,2} \tilde{\eta}_{s}^{1,1} \tilde{\eta}_{s}^{1,2} \\
& \times \prod_{i=1}^{n} \delta^{2}\left(\sum_{j \neq i} \frac{\left\langle u_{j} u_{i}\right\rangle}{\sigma_{j i}} \tilde{\eta}_{j}^{1, I}-\tilde{\eta}_{i}^{1, I} v_{i}-\tilde{\eta}_{i}^{1, I}\right) \prod_{i=1}^{n} \delta^{2}\left(\sum_{j \neq i} \frac{\left\langle u_{j} u_{i}\right\rangle}{\sigma_{j i}} \eta_{j}^{1, I}-\eta_{i}^{1, I} v_{i}-\eta_{i}^{1, I}\right)
\end{aligned}
$$

where $r(\mathrm{~s})$ range over the positive (negative) helicity gravitons and $t$ ranges over the dilaton states. Despite the various ingredients in the formula, the implementation in Mathematica is relatively fast. For instance, we are mostly interested in the pure dilaton case as it enables us to check the exponentials in (3.30): for $n=5$, that is $A_{5}^{\left(\frac{1}{2}, \frac{1}{2}\right)}\left(W_{1} \phi_{2} \phi_{3} \phi_{4} \bar{W}_{5}\right)$, the computation of $J_{F}^{s=1}$ is readily automated and takes about 15 minutes to perform.

Open Access. This article is distributed under the terms of the Creative Commons Attribution License (CC-BY 4.0), which permits any use, distribution and reproduction in any medium, provided the original author(s) and source are credited.

\section{References}

[1] Z. Bern, J.J.M. Carrasco and H. Johansson, New relations for gauge-theory amplitudes, Phys. Rev. D 78 (2008) 085011 [arXiv:0805.3993] [INSPIRE].

[2] Z. Bern, J.J.M. Carrasco and H. Johansson, Perturbative quantum gravity as a double copy of gauge theory, Phys. Rev. Lett. 105 (2010) 061602 [arXiv:1004.0476] [INSPIRE].

[3] Z. Bern, T. Dennen, Y.-T. Huang and M. Kiermaier, Gravity as the square of gauge theory, Phys. Rev. D 82 (2010) 065003 [arXiv:1004.0693] [InSPIRE].

[4] A. Luna et al., Perturbative spacetimes from Yang-Mills theory, JHEP 04 (2017) 069 [arXiv: 1611.07508] [INSPIRE].

\footnotetext{
${ }^{19}$ The package is available at http://library.wolfram.com/infocenter/MathSource/683.
} 
[5] R. Monteiro and D. O'Connell, The kinematic algebras from the scattering equations, JHEP 03 (2014) 110 [arXiv:1311.1151] [INSPIRE].

[6] A. Luna, R. Monteiro, D. O'Connell and C.D. White, The classical double copy for Taub-NUT spacetime, Phys. Lett. B $\mathbf{7 5 0}$ (2015) 272 [arXiv:1507.01869] [INSPIRE].

[7] G. Cardoso, S. Nagy and S. Nampuri, Multi-centered $N=2$ BPS black holes: a double copy description, JHEP 04 (2017) 037 [arXiv:1611.04409] [INSPIRE].

[8] G.L. Cardoso, S. Nagy and S. Nampuri, A double copy for $N=2$ supergravity: a linearised tale told on-shell, JHEP 10 (2016) 127 [arXiv:1609.05022] [INSPIRE].

[9] M. Carrillo-González, R. Penco and M. Trodden, The classical double copy in maximally symmetric spacetimes, JHEP 04 (2018) 028 [arXiv:1711.01296] [INSPIRE].

[10] A. Luna, R. Monteiro, I. Nicholson and D. O'Connell, Type D spacetimes and the Weyl double copy, Class. Quant. Grav. 36 (2019) 065003 [arXiv: 1810.08183] [InSPIRE].

[11] M. Carrillo González, B. Melcher, K. Ratliff, S. Watson and C.D. White, The classical double copy in three spacetime dimensions, JHEP 07 (2019) 167 [arXiv:1904.11001] [INSPIRE].

[12] N. Arkani-Hamed, Y.-T. Huang and D. O'Connell, Kerr black holes as elementary particles, JHEP 01 (2020) 046 [arXiv:1906.10100] [INSPIRE].

[13] R. Monteiro and D. O'Connell, The kinematic algebra from the self-dual sector, JHEP 07 (2011) 007 [arXiv: 1105.2565] [INSPIRE].

[14] N.E.J. Bjerrum-Bohr, P.H. Damgaard, R. Monteiro and D. O'Connell, Algebras for amplitudes, JHEP 06 (2012) 061 [arXiv:1203.0944] [INSPIRE].

[15] W. Cho and K. Lee, Heterotic Kerr-Schild double field theory and classical double copy, JHEP 07 (2019) 030 [arXiv:1904.11650] [INSPIRE].

[16] G. Chen, H. Johansson, F. Teng and T. Wang, On the kinematic algebra for BCJ numerators beyond the MHV sector, JHEP 11 (2019) 055 [arXiv: 1906.10683] [INSPIRE].

[17] A. Anastasiou, L. Borsten, M.J. Duff, L.J. Hughes and S. Nagy, Yang-Mills origin of gravitational symmetries, Phys. Rev. Lett. 113 (2014) 231606 [arXiv:1408.4434] [INSPIRE].

[18] A. Anastasiou, L. Borsten, M.J. Duff, S. Nagy and M. Zoccali, Gravity as gauge theory squared: a ghost story, Phys. Rev. Lett. 121 (2018) 211601 [arXiv: 1807.02486] [INSPIRE].

[19] S. Mizera and B. Skrzypek, Perturbiner methods for effective field theories and the double copy, JHEP 10 (2018) 018 [arXiv:1809.02096] [INSPIRE].

[20] A. Luna, I. Nicholson, D. O'Connell and C.D. White, Inelastic black hole scattering from charged scalar amplitudes, JHEP 03 (2018) 044 [arXiv:1711.03901] [INSPIRE].

[21] A. Luna, R. Monteiro, I. Nicholson, D. O'Connell and C.D. White, The double copy: Bremsstrahlung and accelerating black holes, JHEP 06 (2016) 023 [arXiv:1603.05737] [INSPIRE].

[22] W.D. Goldberger and A.K. Ridgway, Radiation and the classical double copy for color charges, Phys. Rev. D 95 (2017) 125010 [arXiv:1611.03493] [INSPIRE].

[23] W.D. Goldberger, S.G. Prabhu and J.O. Thompson, Classical gluon and graviton radiation from the bi-adjoint scalar double copy, Phys. Rev. D 96 (2017) 065009 [arXiv:1705.09263] [INSPIRE]. 
[24] W.D. Goldberger and A.K. Ridgway, Bound states and the classical double copy, Phys. Rev. D 97 (2018) 085019 [arXiv: 1711.09493] [INSPIRE].

[25] D. Chester, Radiative double copy for Einstein-Yang-Mills theory, Phys. Rev. D 97 (2018) 084025 [arXiv: 1712.08684] [INSPIRE].

[26] W.D. Goldberger, J. Li and S.G. Prabhu, Spinning particles, axion radiation, and the classical double copy, Phys. Rev. D 97 (2018) 105018 [arXiv:1712.09250] [InSPIRE].

[27] J. Li and S.G. Prabhu, Gravitational radiation from the classical spinning double copy, Phys. Rev. D 97 (2018) 105019 [arXiv:1803.02405] [INSPIRE].

[28] C.-H. Shen, Gravitational radiation from color-kinematics duality, JHEP 11 (2018) 162 [arXiv: 1806. 07388] [INSPIRE].

[29] J. Plefka, J. Steinhoff and W. Wormsbecher, Effective action of dilaton gravity as the classical double copy of Yang-Mills theory, Phys. Rev. D 99 (2019) 024021 [arXiv: 1807.09859] [INSPIRE].

[30] J. Plefka, C. Shi, J. Steinhoff and T. Wang, Breakdown of the classical double copy for the effective action of dilaton-gravity at NNLO, Phys. Rev. D 100 (2019) 086006 [arXiv: 1906.05875] [INSPIRE].

[31] A.P.V. and A. Manu, Classical double copy from color kinematics duality: a proof in the soft limit, Phys. Rev. D 101 (2020) 046014 [arXiv:1907.10021] [INSPIRE].

[32] Z. Bern, C. Cheung, R. Roiban, C.-H. Shen, M.P. Solon and M. Zeng, Black hole binary dynamics from the double copy and effective theory, JHEP 10 (2019) 206 [arXiv: 1908.01493] [INSPIRE].

[33] M.-Z. Chung, Y.-T. Huang and J.-W. Kim, Classical potential for general spinning bodies, JHEP 09 (2020) 074 [arXiv: 1908.08463] [INSPIRE].

[34] H. Johansson and A. Ochirov, Pure gravities via color-kinematics duality for fundamental matter, JHEP 11 (2015) 046 [arXiv: 1407.4772] [INSPIRE].

[35] H. Johansson and A. Ochirov, Color-kinematics duality for QCD amplitudes, JHEP 01 (2016) 170 [arXiv: 1507.00332] [INSPIRE].

[36] L. de la Cruz, A. Kniss and S. Weinzierl, Proof of the fundamental BCJ relations for QCD amplitudes, JHEP 09 (2015) 197 [arXiv: 1508.01432] [INSPIRE].

[37] L. de la Cruz, A. Kniss and S. Weinzierl, Double copies of fermions as matter that interacts only gravitationally, Phys. Rev. Lett. 116 (2016) 201601 [arXiv:1601.04523] [INSPIRE].

[38] R.W. Brown and S.G. Naculich, KLT-type relations for QCD and bicolor amplitudes from color-factor symmetry, JHEP 03 (2018) 057 [arXiv: 1802.01620] [INSPIRE].

[39] J. Plefka and W. Wormsbecher, New relations for graviton-matter amplitudes, Phys. Rev. D 98 (2018) 026011 [arXiv: 1804.09651] [INSPIRE].

[40] M. Chiodaroli, M. Günaydin, H. Johansson and R. Roiban, Complete construction of magical, symmetric and homogeneous $N=2$ supergravities as double copies of gauge theories, Phys. Rev. Lett. 117 (2016) 011603 [arXiv: 1512.09130] [INSPIRE].

[41] A. Anastasiou, L. Borsten, M.J. Duff, A. Marrani, S. Nagy and M. Zoccali, Are all supergravity theories Yang-Mills squared?, Nucl. Phys. B 934 (2018) 606 [arXiv: 1707.03234] [INSPIRE]. 
[42] M. Chiodaroli, Q. Jin and R. Roiban, Color/kinematics duality for general Abelian orbifolds of $N=4$ super Yang-Mills theory, JHEP 01 (2014) 152 [arXiv:1311.3600] [INSPIRE].

[43] Y.-T. Huang and H. Johansson, Equivalent D $=3$ supergravity amplitudes from double copies of three-algebra and two-algebra gauge theories, Phys. Rev. Lett. 110 (2013) 171601 [arXiv: 1210.2255] [INSPIRE].

[44] Y.-T. Huang, H. Johansson and S. Lee, On three-algebra and bi-fundamental matter amplitudes and integrability of supergravity, JHEP 11 (2013) 050 [arXiv:1307.2222] [INSPIRE].

[45] M. Chiodaroli, M. Günaydin, H. Johansson and R. Roiban, Spontaneously broken Yang-Mills-Einstein supergravities as double copies, JHEP 06 (2017) 064 [arXiv: 1511.01740] [INSPIRE].

[46] M. Chiodaroli, M. Günaydin, H. Johansson and R. Roiban, Gauged supergravities and spontaneous supersymmetry breaking from the double copy construction, Phys. Rev. Lett. 120 (2018) 171601 [arXiv: 1710.08796] [INSPIRE].

[47] Y.F. Bautista and A. Guevara, From scattering amplitudes to classical physics: universality, double copy and soft theorems, arXiv:1903.12419 [INSPIRE].

[48] D. Neill and I.Z. Rothstein, Classical space-times from the S matrix, Nucl. Phys. B 877 (2013) 177 [arXiv: 1304.7263] [inSPIRE].

[49] Z. Bern, C. Cheung, R. Roiban, C.-H. Shen, M.P. Solon and M. Zeng, Scattering amplitudes and the conservative Hamiltonian for binary systems at third post-Minkowskian order, Phys. Rev. Lett. 122 (2019) 201603 [arXiv:1901.04424] [INSPIRE].

[50] A. Guevara, A. Ochirov and J. Vines, Scattering of spinning black holes from exponentiated soft factors, JHEP 09 (2019) 056 [arXiv: 1812.06895] [INSPIRE].

[51] M.-Z. Chung, Y.-T. Huang, J.-W. Kim and S. Lee, The simplest massive S-matrix: from minimal coupling to black holes, JHEP 04 (2019) 156 [arXiv:1812.08752] [INSPIRE].

[52] A. Guevara, A. Ochirov and J. Vines, Black-hole scattering with general spin directions from minimal-coupling amplitudes, Phys. Rev. D 100 (2019) 104024 [arXiv:1906.10071] [INSPIRE].

[53] N. Arkani-Hamed, T.-C. Huang and Y.-T. Huang, Scattering amplitudes for all masses and spins, JHEP 11 (2021) 070 [arXiv: 1709.04891] [INSPIRE].

[54] S. Weinberg, Photons and gravitons in S-matrix theory: derivation of charge conservation and equality of gravitational and inertial mass, Phys. Rev. 135 (1964) B1049 [INSPIRE].

[55] J. Vines, Scattering of two spinning black holes in post-Minkowskian gravity, to all orders in spin, and effective-one-body mappings, Class. Quant. Grav. 35 (2018) 084002 [arXiv: 1709.06016] [INSPIRE].

[56] B.R. Holstein, Factorization in graviton scattering and the 'natural' value of the g-factor, gr-qc/0607058 [INSPIRE].

[57] B.R. Holstein, How large is the 'natural' magnetic moment?, Am. J. Phys. 74 (2006) 1104 [hep-ph/0607187] [INSPIRE].

[58] N.E.J. Bjerrum-Bohr, J.F. Donoghue and P. Vanhove, On-shell techniques and universal results in quantum gravity, JHEP 02 (2014) 111 [arXiv:1309.0804] [INSPIRE]. 
[59] S. Weinberg, Dynamic and algebraic symmetries, in Lectures on elementary particles and quantum field theory, volume 1, S. Deser, M. Grisaru and H. Pendleton eds., MIT Press, Cambridge, MA, U.S.A. (1970), pg. 283.

[60] S. Ferrara, M. Porrati and V.L. Telegdi, $g=2$ as the natural value of the tree-level gyromagnetic ratio of elementary particles, Phys. Rev. D 46 (1992) 3529 [InSPIRE].

[61] A. Cucchieri, M. Porrati and S. Deser, Tree level unitarity constraints on the gravitational couplings of higher spin massive fields, Phys. Rev. D 51 (1995) 4543 [hep-th/9408073] [INSPIRE].

[62] B.A. Campbell, N. Kaloper and K.A. Olive, Classical hair for Kerr-Newman black holes in string gravity, Phys. Lett. B 285 (1992) 199 [INSPIRE].

[63] B.A. Campbell, N. Kaloper, R. Madden and K.A. Olive, Physical properties of four-dimensional superstring gravity black hole solutions, Nucl. Phys. B 399 (1993) 137 [hep-th/9301129] [INSPIRE].

[64] D. Garfinkle, G.T. Horowitz and A. Strominger, Charged black holes in string theory, Phys. Rev. D 43 (1991) 3140 [Erratum ibid. 45 (1992) 3888] [INSPIRE].

[65] G.W. Gibbons, Antigravitating black hole solitons with scalar hair in $N=4$ supergravity, Nucl. Phys. B 207 (1982) 337 [INSPIRE].

[66] F. Cachazo, S. He and E.Y. Yuan, Scattering of massless particles in arbitrary dimensions, Phys. Rev. Lett. 113 (2014) 171601 [arXiv:1307.2199] [InSPIRE].

[67] F. Cachazo, S. He and E.Y. Yuan, Scattering of massless particles: scalars, gluons and gravitons, JHEP 07 (2014) 033 [arXiv: 1309.0885] [INSPIRE].

[68] F. Cachazo, S. He and E.Y. Yuan, Einstein-Yang-Mills scattering amplitudes from scattering equations, JHEP 01 (2015) 121 [arXiv:1409.8256] [INSPIRE].

[69] F. Cachazo, S. He and E.Y. Yuan, Scattering equations and matrices: from Einstein to Yang-Mills, DBI and NLSM, JHEP 07 (2015) 149 [arXiv:1412.3479] [INSPIRE].

[70] F. Cachazo, A. Guevara, M. Heydeman, S. Mizera, J.H. Schwarz and C. Wen, The S matrix of $6 D$ super Yang-Mills and maximal supergravity from rational maps, JHEP 09 (2018) 125 [arXiv: 1805.11111] [INSPIRE].

[71] Y. Geyer and L. Mason, Polarized scattering equations for $6 D$ superamplitudes, Phys. Rev. Lett. 122 (2019) 101601 [arXiv: 1812.05548] [INSPIRE].

[72] J.H. Schwarz and C. Wen, Unified formalism for $6 D$ superamplitudes based on a symplectic Grassmannian, JHEP 08 (2019) 125 [arXiv:1907.03485] [INSPIRE].

[73] R.-G. Cai and Y.S. Myung, Black holes in the Brans-Dicke-Maxwell theory, Phys. Rev. D 56 (1997) 3466 [gr-qc/9702037] [INSPIRE].

[74] E. Cremmer, J. Scherk and S. Ferrara, SU(4) invariant supergravity theory, Phys. Lett. B 74 (1978) 61 [INSPIRE].

[75] A.K. Das, SO(4) invariant extended supergravity, Phys. Rev. D 15 (1977) 2805 [inSPIRE].

[76] Z. Bern, C. Boucher-Veronneau and H. Johansson, $N \geq 4$ supergravity amplitudes from gauge theory at one loop, Phys. Rev. D 84 (2011) 105035 [arXiv:1107.1935] [INSPIRE].

[77] C. Boucher-Veronneau and L.J. Dixon, $N \geq 4$ supergravity amplitudes from gauge theory at two loops, JHEP 12 (2011) 046 [arXiv:1110.1132] [INSPIRE]. 
[78] D.A. Kosower, B. Maybee and D. O’Connell, Amplitudes, observables, and classical scattering, JHEP 02 (2019) 137 [arXiv:1811.10950] [INSPIRE].

[79] H. Johansson and A. Ochirov, Double copy for massive quantum particles with spin, JHEP 09 (2019) 040 [arXiv:1906.12292] [INSPIRE].

[80] N.E.J. Bjerrum-Bohr, A. Cristofoli, P.H. Damgaard and H. Gomez, Scalar-graviton amplitudes, JHEP 11 (2019) 148 [arXiv:1908.09755] [INSPIRE].

[81] S. Deser and A. Waldron, Inconsistencies of massive charged gravitating higher spins, Nucl. Phys. B 631 (2002) 369 [hep-th/0112182] [InSPIRE].

[82] H. Kawai, D.C. Lewellen and S.H.H. Tye, A relation between tree amplitudes of closed and open strings, Nucl. Phys. B 269 (1986) 1 [INSPIRE].

[83] Z. Bern, L.J. Dixon, M. Perelstein and J.S. Rozowsky, Multileg one loop gravity amplitudes from gauge theory, Nucl. Phys. B 546 (1999) 423 [hep-th/9811140] [INSPIRE].

[84] N.E.J. Bjerrum-Bohr, P.H. Damgaard, B. Feng and T. Sondergaard, Gravity and Yang-Mills amplitude relations, Phys. Rev. D 82 (2010) 107702 [arXiv: 1005.4367] [INSPIRE].

[85] M. Chiodaroli, M. Günaydin, H. Johansson and R. Roiban, Explicit formulae for Yang-Mills-Einstein amplitudes from the double copy, JHEP 07 (2017) 002 [arXiv: 1703.00421] [INSPIRE].

[86] S.G. Naculich, CHY representations for gauge theory and gravity amplitudes with up to three massive particles, JHEP 05 (2015) 050 [arXiv: 1501.03500] [INSPIRE].

[87] R.A. Porto, Post-Newtonian corrections to the motion of spinning bodies in NRGR, Phys. Rev. D 73 (2006) 104031 [gr-qc/0511061] [INSPIRE].

[88] R.A. Porto and I.Z. Rothstein, The hyperfine Einstein-Infeld-Hoffmann potential, Phys. Rev. Lett. 97 (2006) 021101 [gr-qc/0604099] [INSPIRE].

[89] M. Levi and J. Steinhoff, Spinning gravitating objects in the effective field theory in the post-Newtonian scheme, JHEP 09 (2015) 219 [arXiv:1501.04956] [INSPIRE].

[90] M. Levi and J. Steinhoff, Leading order finite size effects with spins for inspiralling compact binaries, JHEP 06 (2015) 059 [arXiv:1410.2601] [INSPIRE].

[91] M. Levi, Effective field theories of post-Newtonian gravity: a comprehensive review, Rept. Prog. Phys. 83 (2020) 075901 [arXiv: 1807.01699] [INSPIRE].

[92] R.A. Porto and I.Z. Rothstein, Spin(1)Spin(2) effects in the motion of inspiralling compact binaries at third order in the post-Newtonian expansion, Phys. Rev. D 78 (2008) 044012 [Erratum ibid. 81 (2010) 029904] [arXiv:0802.0720] [INSPIRE].

[93] R.A. Porto and I.Z. Rothstein, Next to leading order Spin(1)Spin(1) effects in the motion of inspiralling compact binaries, Phys. Rev. D 78 (2008) 044013 [Erratum ibid. 81 (2010) 029905] [arXiv:0804.0260] [INSPIRE].

[94] S. Cotogno, C. Lorcé and P. Lowdon, Poincaré constraints on the gravitational form factors for massive states with arbitrary spin, Phys. Rev. D 100 (2019) 045003 [arXiv: 1905.11969] [INSPIRE].

[95] P. Benincasa and F. Cachazo, Consistency conditions on the S-matrix of massless particles, arXiv:0705.4305 [INSPIRE].

[96] H. Pfister and M. King, The gyromagnetic factor in electrodynamics, quantum theory and general relativity, Class. Quant. Grav. 20 (2002) 205. 
[97] X. Bekaert and N. Boulanger, The unitary representations of the Poincaré group in any spacetime dimension, SciPost Phys. Lect. Notes 30 (2021) 1 [hep-th/0611263] [INSPIRE].

[98] G. Velo and D. Zwanziger, Propagation and quantization of Rarita-Schwinger waves in an external electromagnetic potential, Phys. Rev. 186 (1969) 1337 [inSPIRE].

[99] S. Deser, V. Pascalutsa and A. Waldron, Massive spin 3/2 electrodynamics, Phys. Rev. D 62 (2000) 105031 [hep-th/0003011] [INSPIRE].

[100] I. Cortese, R. Rahman and M. Sivakumar, Consistent non-minimal couplings of massive higher-spin particles, Nucl. Phys. B 879 (2014) 143 [arXiv:1307.7710] [InSPIRE].

[101] M. Porrati, R. Rahman and A. Sagnotti, String theory and the Velo-Zwanziger problem, Nucl. Phys. B 846 (2011) 250 [arXiv:1011.6411] [InSPIRE].

[102] C. Lorce, Electromagnetic properties for arbitrary spin particles. Part 1. Electromagnetic current and multipole decomposition, arXiv:0901.4199 [INSPIRE].

[103] C. Lorce, Electromagnetic properties for arbitrary spin particles: natural electromagnetic moments from light-cone arguments, Phys. Rev. D 79 (2009) 113011 [arXiv:0901.4200] [INSPIRE].

[104] A. Guevara, Holomorphic classical limit for spin effects in gravitational and electromagnetic scattering, JHEP 04 (2019) 033 [arXiv:1706.02314] [INSPIRE].

[105] F. Cachazo and A. Strominger, Evidence for a new soft graviton theorem, arXiv:1404.4091 [INSPIRE].

[106] V. Vaidya, Gravitational spin Hamiltonians from the S matrix, Phys. Rev. D 91 (2015) 024017 [arXiv: 1410.5348 ] [INSPIRE].

[107] B. Maybee, D. O'Connell and J. Vines, Observables and amplitudes for spinning particles and black holes, JHEP 12 (2019) 156 [arXiv:1906.09260] [INSPIRE].

[108] L. Brink, J.H. Schwarz and J. Scherk, Supersymmetric Yang-Mills theories, Nucl. Phys. B 121 (1977) 77 [INSPIRE].

[109] C. Cheung, C.-H. Shen and C. Wen, Unifying relations for scattering amplitudes, JHEP 02 (2018) 095 [arXiv: 1705.03025] [inSPIRE].

[110] C. Cheung, G.N. Remmen, C.-H. Shen and C. Wen, Pions as gluons in higher dimensions, JHEP 04 (2018) 129 [arXiv:1709.04932] [INSPIRE].

[111] M. Chiodaroli, M. Günaydin, H. Johansson and R. Roiban, Scattering amplitudes in $N=2$ Maxwell-Einstein and Yang-Mills/Einstein supergravity, JHEP 01 (2015) 081 [arXiv: 1408.0764] [INSPIRE].

[112] F.J. Belinfante, Intrinsic magnetic moment of elementary particles of spin $\frac{3}{2}$, Phys. Rev. 92 (1953) 997 [INSPIRE].

[113] J. Polchinski, String theory: volume 1, an introduction to the bosonic string, Cambridge University Press, Cambridge, U.K. (2011).

[114] M. Frau, I. Pesando, S. Sciuto, A. Lerda and R. Russo, Scattering of closed strings from many D-branes, Phys. Lett. B 400 (1997) 52 [hep-th/9702037] [INSPIRE].

[115] A. Sheykhi and H. Alavirad, Topological black holes in Brans-Dicke-Maxwell theory, Int. J. Mod. Phys. D 18 (2009) 1773 [arXiv:0809.0555] [INSPIRE]. 
[116] J.H. Horne and G.T. Horowitz, Rotating dilaton black holes, Phys. Rev. D 46 (1992) 1340 [hep-th/9203083] [INSPIRE].

[117] C. Pacilio, Scalar charge of black holes in Einstein-Maxwell-dilaton theory, Phys. Rev. D 98 (2018) 064055 [arXiv: 1806.10238] [InSPIRE].

[118] T. Melia, Dyck words and multiquark primitive amplitudes, Phys. Rev. D 88 (2013) 014020 [arXiv:1304.7809] [INSPIRE].

[119] T. Melia, Getting more flavor out of one-flavor QCD, Phys. Rev. D 89 (2014) 074012 [arXiv: 1312.0599] [INSPIRE].

[120] T. Melia, Proof of a new colour decomposition for QCD amplitudes, JHEP 12 (2015) 107 [arXiv: 1509.03297] [INSPIRE].

[121] A. Ochirov and B. Page, Multi-quark colour decompositions from unitarity, JHEP 10 (2019) 058 [arXiv: 1908.02695] [INSPIRE].

[122] G. Kälin, G. Mogull and A. Ochirov, Two-loop $N=2 S Q C D$ amplitudes with external matter from iterated cuts, JHEP 07 (2019) 120 [arXiv:1811.09604] [INSPIRE].

[123] H. Johansson, G. Kälin and G. Mogull, Two-loop supersymmetric QCD and half-maximal supergravity amplitudes, JHEP 09 (2017) 019 [arXiv:1706.09381] [INSPIRE].

[124] G. Kälin, Cyclic Mario worlds - color-decomposition for one-loop QCD, JHEP 04 (2018) 141 [arXiv: 1712.03539] [INSPIRE].

[125] B.R. Holstein and A. Ross, Spin effects in long range electromagnetic scattering, arXiv:0802.0715 [INSPIRE].

[126] R. Monteiro, D. O'Connell and C.D. White, Black holes and the double copy, JHEP 12 (2014) 056 [arXiv: 1410.0239] [INSPIRE].

[127] P.H. Damgaard, K. Haddad and A. Helset, Heavy black hole effective theory, JHEP 11 (2019) 070 [arXiv: 1908.10308] [INSPIRE].

[128] B.R. Holstein and A. Ross, Spin effects in long range gravitational scattering, arXiv: 0802.0716 [INSPIRE].

[129] C. Cheung, I.Z. Rothstein and M.P. Solon, From scattering amplitudes to classical potentials in the post-Minkowskian expansion, Phys. Rev. Lett. 121 (2018) 251101 [arXiv: 1808.02489] [INSPIRE].

[130] W.T. Emond and N. Moynihan, Scattering amplitudes, black holes and leading singularities in cubic theories of gravity, JHEP 12 (2019) 019 [arXiv:1905.08213] [INSPIRE].

[131] A. Brandhuber and G. Travaglini, On higher-derivative effects on the gravitational potential and particle bending, JHEP 01 (2020) 010 [arXiv:1905.05657] [INSPIRE].

[132] D.Z. Freedman and P.A.V. Proeyen, Supergravity, Cambridge University Press, Cambridge, U.K. (2012).

[133] H. Nicolai and P.K. Townsend, $N=3$ supersymmetry multiplets with vanishing trace anomaly: building blocks of the $N>3$ supergravities, Phys. Lett. B 98 (1981) 257 [INSPIRE].

[134] S. He and Y. Zhang, Connected formulas for amplitudes in standard model, JHEP 03 (2017) 093 [arXiv: 1607. 02843] [inSPIRE].

[135] F. Cachazo, S. He and E.Y. Yuan, Scattering equations and Kawai-Lewellen-Tye orthogonality, Phys. Rev. D 90 (2014) 065001 [arXiv:1306.6575] [InSPIRE]. 
[136] S. Mizera, Aspects of scattering amplitudes and moduli space localization, Ph.D. thesis, Inst. Advanced Study, Princeton, NJ, U.S.A. (2020) [arXiv: 1906. 02099] [INSPIRE].

[137] M. Heydeman, J.H. Schwarz and C. Wen, M5-brane and D-brane scattering amplitudes, JHEP 12 (2017) 003 [arXiv:1710.02170] [INSPIRE].

[138] E. Witten, Perturbative gauge theory as a string theory in twistor space, Commun. Math. Phys. 252 (2004) 189 [hep-th/0312171] [INSPIRE].

[139] R. Roiban, M. Spradlin and A. Volovich, A googly amplitude from the B model in twistor space, JHEP 04 (2004) 012 [hep-th/0402016] [INSPIRE].

[140] R. Roiban, M. Spradlin and A. Volovich, On the tree level S matrix of Yang-Mills theory, Phys. Rev. D 70 (2004) 026009 [hep-th/0403190] [InSPIRE].

[141] Y.-T. Huang, Non-chiral S-matrix of $N=4$ super Yang-Mills, arXiv:1104.2021 [INSPIRE].

[142] L.J. Dixon, J.M. Henn, J. Plefka and T. Schuster, All tree-level amplitudes in massless QCD, JHEP 01 (2011) 035 [arXiv: 1010.3991] [INSPIRE].

[143] Y. Geyer, A.E. Lipstein and L.J. Mason, Ambitwistor strings in four dimensions, Phys. Rev. Lett. 113 (2014) 081602 [arXiv:1404.6219] [INSPIRE]. 